\title{
THE 3D-INDEX AND NORMAL SURFACES
}

\author{
STAVROS GAROUFALIDIS, CRAIG D. HODGSON, NEIL R. HOFFMAN, \\ AND J. HYAM RUBINSTEIN
}

\begin{abstract}
Dimofte, Gaiotto and Gukov introduced a powerful invariant, the 3D-index, associated to a suitable ideal triangulation of a 3-manifold with torus boundary components. The 3D-index is a collection of formal power series in $q^{1 / 2}$ with integer coefficients. Our goal is to explain how the 3D-index is a generating series of normal surfaces associated to the ideal triangulation. This shows a connection of the 3D-index with classical normal surface theory, and fulfills a dream of constructing topological invariants of 3-manifolds using normal surfaces.
\end{abstract}

\section{Contents}

1. Introduction 2

Acknowledgements 3

2. The tetrahedral index 4

3. Notation and conventions for triangulated 3-manifolds 5

3.1. Gluing equations 5

3.2. Generalised angle structures 6

4. The 3D-index of an ideal triangulation 6

5. Dependence on the choice of triangulation 8

6. Some Q-normal surface theory 10

6.1. Construction of spun normal surfaces 11

6.2. Geometric generators for Q-normal classes 13

7. Combinatorics of ideal triangulations and integer normal surface theory 16

7.1. Geometric generators for integer Q-normal classes 19

8. The 3D-index via normal surfaces 20

8.1. Convergence of the index sum 21

8.2. Non 1-efficient ideal triangulations 24

9. Invariance under 2-3 and 0-2 moves 25

10. Generalised normal surfaces 27

10.1. Generalised normal and spun normal surfaces 27

$\begin{array}{ll}\text { 10.2. } & \text { Degrees of index terms } \\ \text { 10.3. } & 29\end{array}$

10.3. Normalisation and barriers 30

11. Some examples 35

11.1. Solid torus 35

11.2. Trefoil complement $\quad 35$

11.3. $\quad \mathbf{T}^{\mathbf{2}} \times \mathbf{I} \quad 36$

11.4. A toroidal example 36 
11.5. $\mathrm{m} 009$

12. Notes on connectedness of the 1-efficient Pachner Graph 39

12.1. A census of ideal triangulations 41

12.2. Two tetrahedron ideal triangulations 42

12.3. Angle Structures, 1-efficiency and Pachner moves 44

12.4. Connected components of normal surfaces 45

Appendix A. Generalised angle structures and the Euler characteristic 50

A.1. Euler characteristic from generalised angle structures $\quad 51$

$\begin{array}{ll}\text { References } & 55\end{array}$

\section{INTRODUCTION}

Recently, the physicists Dimofte, Gaiotto and Gukov [DGG13, DGG14] introduced a powerful new invariant for a compact orientable 3 -manifold $M$ with non-empty boundary $\partial M$ consisting of tori, called the $3 D$-index. This invariant arises from a gauge theory with $N=2$ supersymmetry under a low energy limit, and seems to contain a great deal of information about the geometry and topology of the manifold. The 3D-index is a collection of $q$-series, i.e. formal Laurent series in $q^{1 / 2}$, defined as an infinite sum over integer weights attached to the edges of a chosen ideal triangulation $\mathcal{T}$ of $M$. The $q$-series $I_{\mathcal{T}}(b)$ are parametrised by a choice of peripheral homology class $b \in H_{1}(\partial M ; \mathbb{Z})$.

Our goal is to explain how the 3D-index can be viewed as a generating series of normal surfaces on $\mathcal{T}$; see Definition 8.1 and Corollary 8.2 below. Normal surfaces depend heavily on the ideal triangulation whereas the $3 \mathrm{D}$-index should not. Our results show a connection of the 3D-index with classical normal surface theory, and fulfill a 'folk dream' of constructing topological invariants of 3-manifolds using normal surfaces.

In this paper we first recall how the 3D-index of an ideal triangulation is defined, following [GHRS15], and discuss some of its key properties. Throughout the paper, by a triangulation of a compact 3-manifold we mean an ideal triangulations in the sense of Thurston [Thu77] (see also [NZ85, Neu92]). Physics predicts that the 3D-index should give a topological invariant of the underlying manifold $M$, but this is not known in general. In fact, the sum defining the 3D-index need not even converge (as a formal Laurent series) for all ideal triangulations $\mathcal{T}$. But we can characterise the good triangulations using the normal surface theory developed by Haken [Hak61b, Hak61a]. (Haken's theory applies to ideal triangulations, when restricting to closed normal surfaces.) In fact, the work of [GHRS15] shows the index sum for $I_{\mathcal{T}}(b)$ converges for all $b \in H_{1}(\partial M ; \mathbb{Z})$ if and only if the triangulation is 1-efficient, i.e. contains no embedded closed normal surfaces $S$ of Euler characteristic $\chi(S) \geq 0$ except peripheral tori.

By work of Matveev and Piergallini, (see [Mat87, Mat03, Pie88, BP97]), any two ideal triangulations $\mathcal{T}, \mathcal{T}^{\prime}$ (with at least 2 tetrahedra) of a given compact 3-manifold $M$ with nonempty boundary can be connected by a sequence of 2-3 and 3-2 Pachner moves. Similarly, we can also consider 0-2 and 2-0 moves on a triangulation as shown in Figure 4 below.

The work of [Gar15, GHRS15] shows that the index is invariant under 2-3/3-2 and 0-2/2-0 moves provided all ideal triangulations involved are 1-efficient. However it is not currently 
known whether any two 1-efficient triangulations of a given manifold can be connected by 2-3/3-2 and 0-2/2-0 moves preserving 1-efficiency.

We then give a new formulation of the definition of index as a sum over certain singular normal surfaces. The minimum degree of each term in this sum then has a simple geometric interpretation involving the Euler characteristic and the number of double arcs of the corresponding surface. This leads to new, more direct proofs of results on the convergence of the series for the 3D-index. We also introduce a class of embedded generalised normal surfaces, and show how to express the index as a sum over terms corresponding to these embedded surfaces.

Next we show that the work of Neumann [Neu92] on combinatorics of ideal triangulations can be reinterpreted to give a precise description of the set $Q(\mathcal{T} ; \mathbb{Z})$ of all integer solutions to the $Q$-matching equations of Tollefson [Tol98]. Each such solution $S$ represents a (possibly singular) spun normal surface [KR04, Til08] with well-defined homology class $[S]_{2} \in H_{2}(M, \partial M ; \mathbb{Z} / 2 \mathbb{Z})$ and boundary $[\partial S] \in H_{1}(\partial M ; \mathbb{Z})$ such that the mod 2 reduction of $[\partial S]$ is the image of $[S]$ under the boundary map $\partial_{*}: H_{1}(M ; \mathbb{Z} / 2 \mathbb{Z}) \rightarrow H_{1}(\partial M ; \mathbb{Z} / 2 \mathbb{Z})$. Further, each pair $(a, b) \in H_{2}(M, \partial M ; \mathbb{Z} / 2 \mathbb{Z}) \times H_{1}(\partial M ; \mathbb{Z})$ such that $\partial_{*} a=b \bmod 2$ arises in this way, and the $Q$-normal classes $S$ with $[S]_{2}=0$ and $[\partial S]=0$ are the integer linear combinations of the "edge solutions" and the "tetrahedral solutions" constructed by Kang-Rubinstein [KR04].

We then show that the definition of 3D-index can be extended to give a $q$-series $I_{\mathcal{T}}^{a}(b)$ for each $(a, b)$ as above; this is a sum over $Q$-normal classes $S$ with $[S]=a$ and $[\partial S]=b$ modulo tetrahedral solutions. The previous definition of 3D-index only applies to the cases where $a=0$ and $b \in H_{1}(\partial M ; 2 \mathbb{Z})$; we have $I_{\mathcal{T}}^{0}(b)=I_{\mathcal{T}}(2 b)$ in the notation of [GHRS15]. Here $I_{\mathcal{T}}^{0}(b)$ is also defined for $b \in \mathcal{K}=\operatorname{Ker}\left(H_{1}(\partial M ; \mathbb{Z}) \rightarrow H_{1}(M ; \mathbb{Z} / 2 \mathbb{Z})\right)$.

We then give some computations of the 3D-index, including an example showing that that the series for the 3D-index $I_{\mathfrak{T}}^{a}(b)$ can sometimes converge for $b \neq 0$ even when the triangulation $\mathcal{T}$ is not 1-efficient (so the series for $I_{\mathcal{T}}^{0}(0)$ does not converge).

We conclude with some discussion of experimental results on the connectedness of the set of 1-efficient ideal triangulations of a given manifold under 2-3/3 and 0-2/2-0 Pachner moves. (Recall that such moves do not change the 3D-index.) In particular, we find examples of 1-efficient triangulations of the solid torus with six tetrahedra that cannot be connected by sequences of 2-3/3-2 moves through 1-efficient triangulations. These triangulations can, however, be connected via 1-efficient triangulations if $0-2$ and 2-0 moves are also allowed; hence they have the same 3D-index.

Finally, the appendix gives some results on generalised angle structures and Euler characteristic for Q-normal surfaces; these extend well-known results of Luo-Tillmann [LT08] for closed normal surfaces.

Acknowledgements. The first author is partially supported by National Science Foundation Grant DMS-14-06419. The last three authors are partially supported by Australian Research Council Discovery Grant DP130103694. The authors wish to thank Tudor Dimofte and Henry Segerman for useful discussions related to this paper. 


\section{THE TETRAHEDRAL INDEX}

The index for a triangulation is built up from the tetrahedron index $I_{\Delta}: \mathbb{Z}^{2} \rightarrow \mathbb{Z}\left[\left[q^{\frac{1}{2}}\right]\right]$, defined for $m, e \in \mathbb{Z}$ by

$$
I_{\Delta}(m, e)(q)=\sum_{n=\max \{0,-e\}}^{\infty}(-1)^{n} \frac{q^{\frac{1}{2} n(n+1)-\left(n+\frac{1}{2} e\right) m}}{(q)_{n}(q)_{n+e}}
$$

where $(q)_{n}=\prod_{i=1}^{n}\left(1-q^{i}\right)$ is the $q$-Pochhammer symbol (by convention $(q)_{0}=1$ ). The index coincides with the coefficients of $z^{e}$ in the generating function

$$
I(m, q, z)=\sum_{e \in \mathbb{Z}} I_{\Delta}(m, e)(q) z^{e}=\frac{\left(q^{-\frac{m}{2}+1} z^{-1} ; q\right)_{\infty}}{\left(q^{-\frac{m}{2}} z ; q\right)_{\infty}}
$$

where

$$
(z ; q)_{\infty}=\prod_{n=0}^{\infty}\left(1-z q^{n}\right) .
$$

(see [Gar15]). It follows that for any fixed $q \in \mathbb{C}$ with $0<|q|<1$, the product for $I(m, q, z)$ converges for all $z \in \mathbb{C}$ with $z \neq 0$, and defines a holomorphic function of $z$ in the annulus $0<|z|<1$ with Laurent series given by $\sum_{e \in \mathbb{Z}} I_{\Delta}(m, e)(q) z^{e}$. by

A more symmetric version of the tetrahedral index was defined in [GHRS15] for $a, b, c \in \mathbb{Z}$

$$
J_{\Delta}(a, b, c)=\left(-q^{\frac{1}{2}}\right)^{-b} I_{\Delta}(b-c, a-b)=\left(-q^{\frac{1}{2}}\right)^{-c} I_{\Delta}(c-a, b-c)=\left(-q^{\frac{1}{2}}\right)^{-a} I_{\Delta}(a-b, c-a) .
$$

Then $J_{\Delta}(a, b, c)$ is invariant under all permutations of its arguments $a, b, c$ and satisfies

$$
J_{\Delta}(a, b, c)=\left(-q^{\frac{1}{2}}\right)^{s} J_{\Delta}(a+s, b+s, c+s) \text { for all } s \in \mathbb{Z} .
$$

In particular, if $a, b \geq 0$ then (1) gives

$$
J_{\Delta}(a, b, 0)=I_{\Delta}(-a, b)=q^{\frac{1}{2} a b}+\text { higher order terms, }
$$

and (5) shows that, in general, the lowest degree term in $J_{\Delta}(a, b, c)$ has $q$-degree

$$
\operatorname{deg} J_{\Delta}(a, b, c)=\frac{1}{2}((a-m)(b-m)+(b-m)(c-m)+(c-m)(a-m)-m)
$$

and coefficient $(-1)^{m}$, where $m=\min \{a, b, c\}$.

The tetrahedral index also satisfies many other interesting algebraic identities including the quadratic identity

$$
\sum_{e \in \mathbb{Z}} I_{\Delta}(m, e) I_{\Delta}(m, e+c) q^{e}=\delta_{c, 0}
$$

the pentagon identity

$$
\begin{aligned}
& \sum_{e_{0} \in \mathbb{Z}} q^{e_{0}} I_{\Delta}\left(m_{1}, x_{1}+e_{0}\right) I_{\Delta}\left(m_{2}, x_{2}+e_{0}\right) I_{\Delta}\left(m_{1}+m_{2}, x_{3}+e_{0}\right) \\
& \quad=q^{-x_{3}} I_{\Delta}\left(m_{1}-x_{2}+x_{3}, x_{1}-x_{3}\right) I_{\Delta}\left(m_{2}-x_{1}+x_{3}, x_{2}-x_{3}\right),
\end{aligned}
$$

and some important recurrence relations. (See [Gar15] for the details.) 


\section{NOtATiOn AND CONVENTIONS FOR TRIANGUlated 3-MANifOldS}

In this paper, we will observe the following conventions and notation.

Let $M$ be a compact orientable 3-manifold with boundary consisting of $r$ tori. Let $\mathcal{T}$ be an ideal triangulation of int $M$ with $n$ ideal tetrahedra $\sigma_{j}$, (i.e. tetrahedra with their vertices removed). Then there are also $n$ edge classes, since $\chi(M)=0$.

3.1. Gluing equations. We now briefly describe how to encode the combinatorics of a triangulation contained in Thurston's gluing equations (compare [Thu77, Chapter 4]), while also including notation for normal disks in a tetrahedron. First, in each tetrahedron there are three pairs of opposite edges, which are disjoint from the three quadrilateral disks from normal surface theory. We label these quadrilaterals by the pair of edges they face, as in Regina [Bur]; Figure 1 shows the quads $q_{A}=q_{A: 01: 23}, q_{A}^{\prime}=q_{A: 02: 13}, \quad q_{A}^{\prime \prime}=q_{A: 03: 12}$ in a tetrahedron labelled $A$. As our discussion unfolds the quadrilaterals will be the focus, however we also provide a labelling for the triangular disks for completeness: $t_{A: j}$ refers the the triangle in tetrahedron $A$ that cuts off vertex $j$.
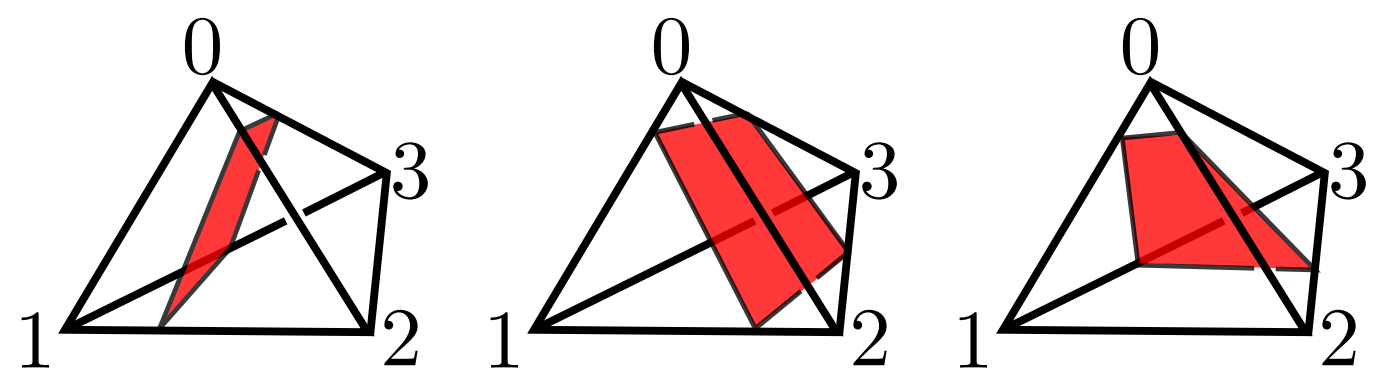

Figure 1. The labelling of the 3 quadrilateral disk types of tetrahedron $A$.

Left: $q_{A}=q_{A: 01: 23}$, centre: $q_{A}^{\prime}=q_{A: 02: 13}$, right: $q_{A}^{\prime \prime}=q_{A: 03: 12}$.

Central to the construction of Thurston's gluing equations is the observation that edges in each tetrahedron form equivalence classes in the triangulation. In Thurston's notes, a set of logarithmic equations is associated to the $n$ edge classes of the triangulation. First, we associate a complex tetrahedral parameter $z_{j}$ to the 01,23 edges in tetrahedron $j, z_{j}^{\prime}=\frac{1}{1-z_{j}}$ to the 02,13 edges and $z_{j}^{\prime \prime}=\frac{z_{j}-1}{z_{j}}$ to the 03,12 edges.

Then we can build the following edge equations:

$$
\sum_{j}\left(a_{i, j} \log \left(z_{j}\right)+b_{i, j} \log \left(z_{j}^{\prime}\right)+c_{i, j} \log \left(z_{j}^{\prime \prime}\right)\right)=0+2 \pi \sqrt{-1} \text { for } i \in\{1, \ldots, n\},
$$

where $a_{i, j}, b_{i, j}, c_{i, j}$ record the number of times the edges opposite quads $q_{j}, q_{j}^{\prime}, q_{j}^{\prime \prime}$ of tetrahedron $j$ appear in the $i$ th edge class. (Our convention is that $\arg \left(z_{j}\right), \arg \left(z_{j}^{\prime}\right), \arg \left(z_{j}^{\prime \prime}\right) \in$ $(-\pi, \pi]$.

Note that the tetrahedral parameters also satisfy the relation

$$
\log \left(z_{j}\right)+\log \left(z_{j}^{\prime}\right)+\log \left(z_{j}^{\prime \prime}\right)=\pi \sqrt{-1} .
$$


We can also build $2 r$ cusp equations using the same tetrahedral parameters:

$$
\sum_{j}\left(a_{i, j} \log \left(z_{j}\right)+b_{i, j} \log \left(z_{j}^{\prime}\right)+c_{i, j} \log \left(z_{j}^{\prime \prime}\right)\right)=0+\pi \sqrt{-1} \text { for } i \in\{n+1, \ldots, n+2 r\},
$$

however here $a_{i, j}, b_{i, j}, c_{i, j}$ record the number of times a peripheral curve winds anti-clockwise (or minus the number of clockwise times) around the edges opposite the quads $q_{j}, q_{j}^{\prime}, q_{j}^{\prime \prime}$ in tetrahedron $j$.

The coefficients in the edge and cusp equations define a $(n+2 r) \times 3 n$ gluing matrix $\left[\begin{array}{lll}a_{i, j} & b_{i, j} & c_{i, j}\end{array}\right]$, which is also given by the gluing_equations() function in SnapPy [CDW].

Throughout the paper, we will write $E_{i}$ for the vector of coefficients in the $i$ th edge equation, and we will also label the cusp equation coefficients coming from the $k$ th cusp by $M_{k}$ and $L_{k}$ and the corresponding curves $\mu_{k}$ and $\lambda_{k}$ where it proves convenient.

3.2. Generalised angle structures. Considering the imaginary parts of above gluing equations allows us to define an angle structure on the triangulation. If we define $\square$ to be the set of quad types in a triangulation $\mathcal{T}$, then a generalised angle structure on $\mathcal{T}$ is a function $\alpha: \square \rightarrow \mathbb{R}$ which assigns an "angle" $\alpha(q) \in \mathbb{R}$ to the pair of edges opposite a quad $q$ and satisfies the set of equations:

$$
\alpha\left(q_{j}\right)+\alpha\left(q_{j}^{\prime}\right)+\alpha\left(q_{j}^{\prime \prime}\right)=\pi \text { for } j \in\{1, \ldots, n\}
$$

and

$$
\sum_{j}\left(a_{i, j} \alpha\left(q_{j}\right)+b_{i, j} \alpha\left(q_{j}^{\prime}\right)+c_{i, j} \alpha\left(q_{j}^{\prime \prime}\right)\right)=2 \pi \text { for } i \in\{1, \ldots, n\}
$$

where $a_{i, j}, b_{i, j}, c_{i, j}$ are defined above. A strict angle structure satisfies the additional condition that $\alpha(q)>0$ for all quads $q$ and a semi angle structure satisfies $\alpha(q) \geq 0$.

A further refinement of generalised angle structures which considers the holonomies of peripheral curves will be defined in Appendix A.

A central theme of this paper is that the entries of the gluing matrix can be interpreted in a number of different and interesting ways. By exploiting these relationships, we are able to better understand the 3D-index of a triangulation, which will be defined in the next section.

\section{The 3D-INDEX OF AN IDEAL TRIANGULATION}

Using the notation from the previous section, we now give a definition of the 3D-index of an ideal triangulation $\mathcal{T}$ as formulated in [GHRS15, section 4.7].

Given $\boldsymbol{k}=\left(k_{1}, \ldots, k_{n}\right) \in \mathbb{Z}^{n}$, we can assign an integer weight $k_{i}$ to the $i$ th edge class. Effectively, this also gives a weight on each edge of each tetrahedron $\sigma_{j}$ in $\mathcal{T}$. Let $a_{j}(\boldsymbol{k}), b_{j}(\boldsymbol{k}), c_{j}(\boldsymbol{k})$ be the sums of weights assigned to the pair of edges in tetrahedron $j$ opposite quads $q_{j}, q_{j}^{\prime}, q_{j}^{\prime \prime}$ respectively. Note that these coefficients are precisely the entries in the linear combination $\sum_{i=1}^{n} k_{i} E_{i}$ of the rows of the gluing matrix corresponding to the edge equations.

Now we define a tetrahedral index associated to the tetrahedron $\sigma_{j}$ by

$$
J\left(\sigma_{j} ; \boldsymbol{k}\right)=J_{\Delta}\left(a_{j}(\boldsymbol{k}), b_{j}(\boldsymbol{k}), c_{j}(\boldsymbol{k})\right) .
$$


and the $3 D$-index $I_{\mathcal{T}}(\mathbf{0})$ of the triangulation $\mathcal{T}$ is defined as

$$
I_{\mathcal{T}}(\mathbf{0})(q)=\sum_{\boldsymbol{k} \in \mathbb{Z}^{n-r}} q^{\sum_{i} k_{i}} \prod_{j=1}^{n} J\left(\sigma_{j} ; \boldsymbol{k}\right),
$$

where the summation is over a (suitable) sublattice $\mathbb{Z}^{n-r} \subset \mathbb{Z}^{n}$, obtained by setting $r$ of the edge weights $k_{i}$ equal to zero. For $r=1$ we can set any $k_{i}=0$; but in general the $r$ edge weights set to zero must be chosen carefully (see [GHRS15, sections 4.5, 4.6]).

More generally, the 3 D-index gives a function $I_{\mathcal{T}}: H_{1}(\partial M ; \mathbb{Z}) \cong \mathbb{Z}^{2 r} \rightarrow \mathbb{Z}\left(\left(q^{\frac{1}{2}}\right)\right)$ defined as follows. Let $\gamma$ be an oriented multicurve on $\partial M$ with no contractible components, such that $\gamma$ is in normal position with respect to the induced triangulation $\mathcal{T}_{\partial}$ of $\partial M$. Then let $\gamma_{h}$ be the component of $\gamma$ in cusp $h$ and express $\gamma_{h}=p_{h} \mu_{h}+q_{h} \lambda_{h}$, where $\mu_{h}$ corresponds to the row $M_{h}$ in the gluing matrix and $\lambda_{h}$ corresponds to $L_{h}$. Now put

$$
J\left(\sigma_{j} ; \boldsymbol{k}, \gamma\right)=J_{\Delta}\left(a_{j}(\boldsymbol{k}, \gamma), b_{j}(\boldsymbol{k}, \gamma), c_{j}(\boldsymbol{k}, \gamma)\right)
$$

where the coefficients $a_{j}(\boldsymbol{k}, \gamma), b_{j}(\boldsymbol{k}, \gamma), c_{j}(\boldsymbol{k}, \gamma)$ are precisely the entries in the linear combination $\sum_{i=1}^{n} k_{i} E_{i}+\sum_{h=1}^{n}\left(p_{h} M_{h}+q_{h} L_{h}\right)$ corresponding to tetrahedron $\sigma_{j}$.

Then the general $3 D$-index of the triangulation $\mathcal{T}$ is defined as

$$
I_{\mathcal{T}}([\gamma])(q)=\sum_{\boldsymbol{k} \in \mathbb{Z}^{n-r}} q^{\sum_{i} k_{i}} \prod_{j} J\left(\sigma_{j} ; \boldsymbol{k}, \gamma\right)
$$

where $[\gamma] \in H_{1}(\partial M ; \mathbb{Z})$ is the homology class of $\gamma$ and the summation is over $\mathbb{Z}^{n-r} \subset \mathbb{Z}^{n}$ as above. (It is shown in [GHRS15] that the sum only depends on the homology class of $\gamma$.)

Example 4.1. For the figure eight knot complement $M$, with the ideal triangulation given by Thurston [Thu77, §4], we have the following induced triangulation on $\partial M$ (as viewed from the cusp).

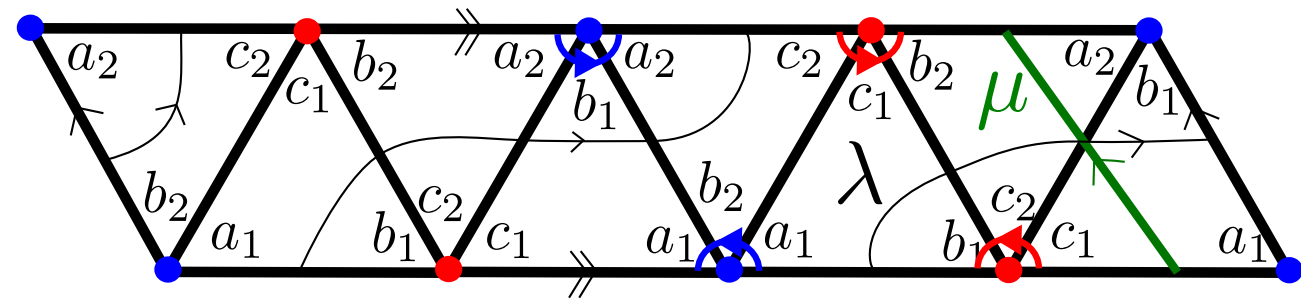

Figure 2. A labelled triangulation of the cusp of the figure 8 knot complement.

From this we can read off the following gluing data.

Choosing generators $\mu, \lambda$ for $H_{1}(\partial M, \mathbb{Z})=\mathbb{Z}^{2}$ corresponding to the standard meridian and longitude, and integer weights $k_{1}=k, k_{2}=0$ on the edges gives 


\begin{tabular}{|c||c|c|c|c|c|c|}
\hline edge/peripheral curve & $a_{1}$ & $b_{1}$ & $c_{1}$ & $a_{2}$ & $b_{2}$ & $c_{2}$ \\
\hline \hline edge 1 (blue) & 2 & 1 & 0 & 2 & 1 & 0 \\
\hline edge 2 (red) & 0 & 1 & 2 & 0 & 1 & 2 \\
\hline meridian $\mu$ & 0 & 0 & 1 & -1 & 0 & 0 \\
\hline longitude $\lambda$ & 0 & 0 & 0 & 2 & 0 & -2 \\
\hline
\end{tabular}

$$
\begin{aligned}
I_{\mathcal{T}}(x \mu+y \lambda) & =\sum_{k \in \mathbb{Z}} q^{k} J_{\Delta}(2 k, k, x) J_{\Delta}(2 k-x+2 y, k,-2 y) \\
& =\sum_{k \in \mathbb{Z}} I_{\Delta}(k-x, k) I_{\Delta}(k+2 y, k-x+2 y) .
\end{aligned}
$$

For example, up to terms of order $q^{10}$, we have

$$
\begin{aligned}
I_{\mathcal{T}}(\mathbf{0}) & =1-2 q-3 q^{2}+2 q^{3}+8 q^{4}+18 q^{5}+18 q^{6}+14 q^{7}-12 q^{8}-52 q^{9}-106 q^{10}+\ldots \\
I_{\mathcal{T}}(\mu) & =2 q-2 q^{2}+2 q^{3}+8 q^{4}+16 q^{5}+16 q^{6}+10 q^{7}-14 q^{8}-52 q^{9}-102 q^{10}+\ldots \\
I_{\mathcal{T}}(2 \mu) & =-q-q^{2}+3 q^{3}+6 q^{4}+12 q^{5}+9 q^{6}+3 q^{7}-19 q^{8}-50 q^{9}-88 q^{10}+\ldots \\
I_{\mathcal{T}}(\lambda) & =q^{3}+2 q^{4}+5 q^{5}+2 q^{6}-3 q^{7}-16 q^{8}-32 q^{9}-52 q^{10}+\ldots \\
I_{\mathcal{T}}(4 \mu+\lambda) & =q-q^{4}-2 q^{5}-5 q^{6}-8 q^{7}-10 q^{8}-11 q^{9}-6 q^{10}+\ldots
\end{aligned}
$$

In this example, it is easy to check that $I_{\mathcal{T}}( \pm x \mu \pm y \lambda)=I_{\mathcal{T}}(x \mu+y \lambda)$, and we can also define the index for $x \in \mathbb{Z}, y \in \frac{1}{2} \mathbb{Z}$, as done in [DGG13]. For example,

$$
\begin{aligned}
I_{\mathcal{T}}\left(\frac{1}{2} \lambda\right) & =-2 q^{3 / 2}+4 q^{7 / 2}+10 q^{9 / 2}+14 q^{11 / 2}+10 q^{13 / 2}-2 q^{15 / 2}-32 q^{17 / 2}-68 q^{19 / 2}+\ldots \\
I_{\mathcal{T}}\left(\mu+\frac{1}{2} \lambda\right) & =-q-q^{2}+2 q^{3}+7 q^{4}+11 q^{5}+11 q^{6}+3 q^{7}-17 q^{8}-49 q^{9}-88 q^{10}+\ldots \\
I_{\mathcal{T}}\left(2 \mu+\frac{1}{2} \lambda\right) & =-q^{1 / 2}+q^{5 / 2}+4 q^{7 / 2}+7 q^{9 / 2}+7 q^{11 / 2}+3 q^{13 / 2}-12 q^{15 / 2}-31 q^{17 / 2}-62 q^{19 / 2}+\ldots
\end{aligned}
$$

\section{Dependence on the ChOiCe of tRiangulation}

Physics predicts that the 3D-index as defined above should give a topological invariant of the underlying manifold $M$, but this is not known in general.

The first difficulty is that the summation in (16) need not even converge (as a formal power series) for all ideal triangulations $\mathcal{T}$. But the good triangulations can be characterised using normal surface theory.

Given a triangulation $\mathcal{T}$ of a 3 -manifold $M$, an embedded surface $S \subset M$ is a normal surface if it intersects each tetrahedron in a finite collection of disjoint normal quadrilaterals ('quads') and triangles as shown in Figure 3. In each tetrahedron there are 4 types of normal triangles and 3 types of normal quads. 

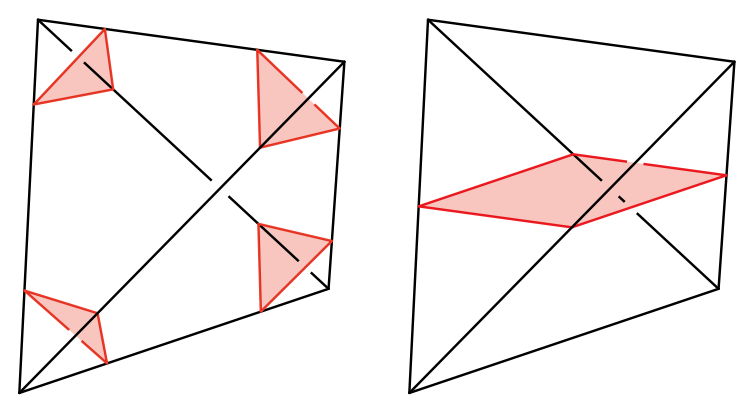

FigurE 3. The four types of triangular disks (left) and one of the three types of quadrilateral disks (right).

Embedded normal surfaces were introduced by Kneser [Kne29] and developed by Haken [Hak61b, Hak61a] to create a normal surface theory which has played a key role in the development of algorithmic 3-manifold topology. Haken observed that (not necessarily embedded) closed normal surfaces are parametrised by vectors of quad numbers and triangle numbers in $\mathbb{Z}^{7 n}$ satisfying linear matching equations. Tollefson [Tol98] showed that the quad numbers are enough to determine a closed normal surface up to multiples of the boundary tori. These give vectors in $\mathbb{Z}^{3 n}$ satisfying linear $Q$-matching equations.

Definition 5.1. [JR03, KR05] An ideal triangulation $\mathcal{T}$ is 1-efficient if

(0) it contains no embedded normal 2-spheres or projective planes, and

(1) the only embedded normal tori or Klein bottles are vertex-linking.

Theorem 5.2. [GHRS15, Theorem 1.2] The sum defining $I_{\mathcal{T}}([\gamma])$ converges for all $[\gamma] \in$ $H_{1}(\partial M ; \mathbb{Z})$ if and only if the sum $I_{\mathcal{T}}(\mathbf{0})$ converges if and only if the ideal triangulation $\mathcal{T}$ is 1-efficient.

We will outline a new, more direct proof of this result in Section 8 below, by rewriting the index as a sum of contributions from normal surfaces.

It is shown in [GHRS15] that 1-efficient triangulations exist for many important classes of cusped 3-manifolds including all hyperbolic manifolds and small Seifert fibre spaces. But they cannot exist for 3-manifolds containing (embedded) essential spheres, projective planes, tori or Klein bottles which are not boundary parallel.

It is known by the work of Matveev and Piergallini (see [Mat87, Mat03, Pie88]) that any two triangulations $\mathcal{T}, \mathcal{T}^{\prime}$ (with at least 2 tetrahedra) of a given closed 3 -manifold $M$ can be connected by a sequence of 2-3 and 3-2 Pachner moves. We can also consider 0-2 and 2-0 moves on triangulations as shown in Figure 4.

The pentagon identity for the tetrahedral index gives the following key property of the 3D-index:

Theorem 5.3 ([Gar15, §6],[GHRS15, Theorem 1.1]). If $\mathcal{T}$ and $\mathcal{T}^{\prime}$ are related by a 2-3 move and both are 1 -efficient, then $I_{\mathcal{T}}=I_{\mathcal{T}^{\prime}}$.

The quadratic identity for the tetrahedral index gives: 


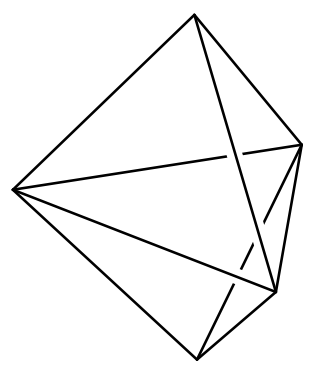

(A) The 2-3 and 3-2 moves.

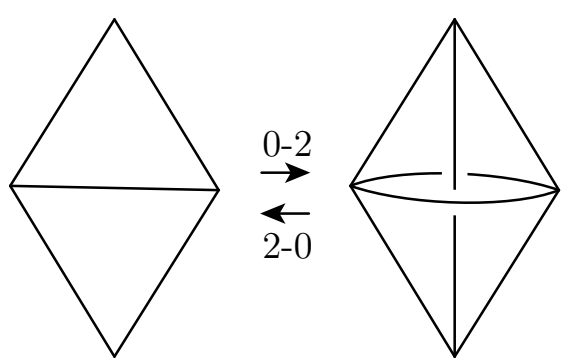

(B) The 0-2 and 2-0 moves.

Figure 4. Moves on (topological) triangulations.

Theorem 5.4. [GHRS15, Theorem 5.1] If $\mathcal{T}$ and $\mathcal{T}^{\prime}$ are ideal triangulations related by a 0-2 move and both are 1 -efficient, then $I_{\mathcal{T}}=I_{\mathcal{T}^{\prime}}$.

It is currently unknown whether any two 1-efficient triangulations of a given cusped manifold are connected by 2-3/3-2 and 0-2/2-0 moves preserving 1-efficiency. See Section 12 for a discussion of some experimental results on this question.

However, using the previous results, we can obtain a topological invariant $I_{M}$ of any cusped hyperbolic 3-manifold $M$ as follows (see [GHRS] for the details).

- Consider the canonical or Epstein-Penner decomposition of $M$ into convex ideal hyperbolic polyhedra (using horoball cusp neighbourhoods of equal volume if $M$ has more than one cusp).

- If the Epstein-Penner decomposition is a triangulation $\mathcal{T}$, then we define $I_{M}=I_{\mathcal{T}}$.

- In general, we can define $I_{M}=I_{\mathcal{T}}$ where $\mathcal{T}$ is any triangulation in a class $\mathcal{E} \mathcal{P}$ consisting of all regular triangulations of the Epstein-Penner cells, with all possible layered triangulations of the bridge regions between any incompatible triangulations of the faces.

- A regular triangulation of a set of points $A=\left\{a_{i}\right\}$ in $\mathbb{R}^{n}$, is obtained by lifting the points vertically to points $\left(a_{i}, t_{i}\right)$ in $\mathbb{R}^{n+1}$, taking the bottom faces of their convex hull in $\mathbb{R}^{n+1}$, and then projecting back to $\mathbb{R}^{n}$. (Use the projective model to extend this definition to $\mathbb{H}^{n}$.)

- All triangulations in class $\mathcal{E} \mathcal{P}$ admit semi-angle structures, hence are 1-efficient.

- Results of Gelfand, Kapranov and Zelevinsky on regular triangulations imply that $\mathcal{E P}$ is connected by sequences of 2-3, 3-2, 0-2 and 2-0 moves, staying within 1-efficient triangulations. But the index is invariant under such moves, so $I(\mathcal{T})$ is the same for all $\mathcal{T}$ in $\mathcal{E P}$.

- Hence we obtain a well-defined invariant for any cusped hyperbolic 3-manifold.

\section{Some Q-NORMal SURFACE TheOrY}

In this section we discuss surfaces in general position with respect to an ideal triangulation. This discussion is similar to the one of Haken who studied and encoded normal surfaces in general position with respect to a triangulation of a closed 3-manifold. A major difference is 
that amongst surfaces in general position with respect to an ideal triangulation is the class of embedded spun normal surfaces (a concept introduced by Thurston, [Thu77, Wal11]), which intersect each tetrahedron in a finite number of quadrilaterals, and a possibly infinite number of triangles. Also, just as in the closed case, a spun normal surface can be reconstructed from its quadrilateral data and the quadrilaterals satisfy the $Q$-matching equations.

Let $M$ be an orientable 3-manifold with boundary consisting of $r$ tori, and let $\mathcal{T}$ be an oriented ideal triangulation with $n$ tetrahedra. Let $\mathcal{E}$ denote the set of edges of $\mathcal{T}$, and recall that $\square$ denotes the set of quad types in the tetrahedra of $\mathcal{T}$. Of course, $|\mathcal{E}|=n$ and $|\square|=3 n$.

Recall that the $Q$-normal surface solution space $Q(\mathcal{T} ; \mathbb{R})$ for $\mathcal{T}$ is a subset of $\mathbb{R}^{\square} \cong \mathbb{R}^{3 n}$ consisting of real quad coordinates assigned to the quad types in $\mathcal{T}$ satisfying the $Q$-matching equations of Tollefson. After choosing a cyclic ordering of quad types in each tetrahedron, compatible with the orientation on $\mathcal{T}$, we can write the quad coordinates of $S \in Q(\mathcal{T} ; \mathbb{R})$ as a vector

$$
S=\left(a_{1}, b_{1}, c_{1}, a_{2}, b_{2}, c_{2}, \ldots, a_{n}, b_{n}, c_{n}\right) \in\left(\mathbb{R}^{3}\right)^{n}=\mathbb{R}^{3 n}
$$

where $\left(a_{j}, b_{j}, c_{j}\right)$ are the quad coordinates of $S$ in tetrahedron $j$.

Let $Q(\mathcal{T} ; \mathbb{Z})=Q(\mathcal{T} ; \mathbb{R}) \cap \mathbb{Z}^{3 n}$ be the sublattice of $Q(\mathcal{T} ; \mathbb{R})$ of integer solutions, and let $Q\left(\mathcal{T} ; \mathbb{Z}_{+}\right)=Q(\mathcal{T} ; \mathbb{Z}) \cap \mathbb{R}_{+}^{3 n}$ denote the set of integer solutions with non-negative quad coordinates. Then each element $x$ of $Q\left(\mathcal{T} ; \mathbb{Z}_{+}\right)$determines a (possibly singular) spun normal surface in $\mathcal{T}$ obtained by taking $x_{A}$ disjoint copies of the quad $q_{A}$ together with additional normal triangles. The resulting surface is a (possibly singular) closed normal surface if only finitely many triangles are added, and is embedded if there is at most one non-zero quad coordinate in each tetrahedron. (See Kang [Kan05], Tillmann [Til08].)

6.1. Construction of spun normal surfaces. Next we give a brief exposition of the construction of a spun normal surface from solutions to the Q-matching equations following [DG12] and [Til08]. (This approach goes back to lectures of W. Thurston and was used by J. Weeks in SnapPea.)

For a spun normal surface, there is a fixed pattern of normal arcs in each ideal triangle, consisting of three infinite families of parallel arcs (one at each corner). Further there is a well-defined "middle" interval in each edge of the triangle separating two of these families. The union of these three intervals with 3 normal arcs form a hexagon, as described in [DG12].

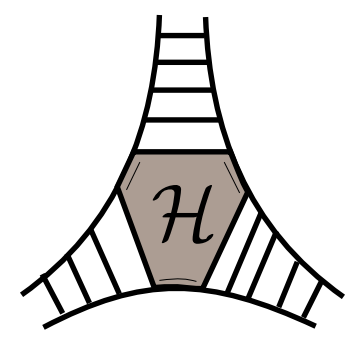

FiguRE 5. The hexagon $\mathcal{H}$ has three arcs which are normal arcs and three arcs (labelled by double lines) that are not normal arcs and represent the "middle" intervals. 
Next consider the arc pattern on the boundary of a tetrahedron: Along each edge, there is an integer "shear" or "shift" parameter which specifies how the adjacent arc patterns fit together, and we choose a sign convention as shown in Figure 6 below (as viewed from outside the tetrahedron).

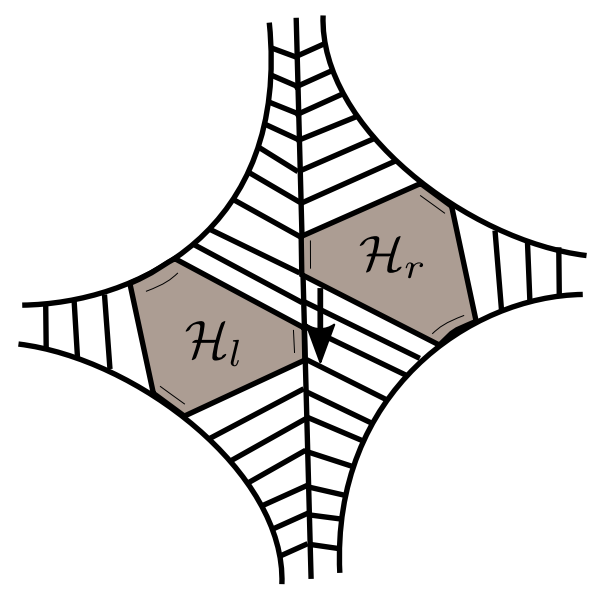

FiguRE 6. A shift of -3 from $\mathcal{H}_{r}$ to $\mathcal{H}_{l}$. (In general, shearing parameters measure the offset from right to left as viewed from outside the tetrahedron.)

We require that the sum of shear parameters along the three edges meeting at a vertex is zero. This means that there is an infinite family of parallel normal triangles at each tetrahedron vertex, and also implies that the shear parameters on opposite edges are equal. With our sign convention, quad coordinates $(a, b, c)$ for quad types $q_{A: 01: 23}, q_{A: 02: 13}, q_{A: 03: 12}$ in a tetrahedron correspond to shear parameters $(c-b, a-c, b-a)$ along the edges of the tetrahedron facing $q_{A: 01: 23}, q_{A: 02: 13}, q_{A: 03: 12}$. (Figure 7 below shows the case of two quads of type $q_{A: 01: 23}$, separating vertices 0,1 from vertices 2,3 .)
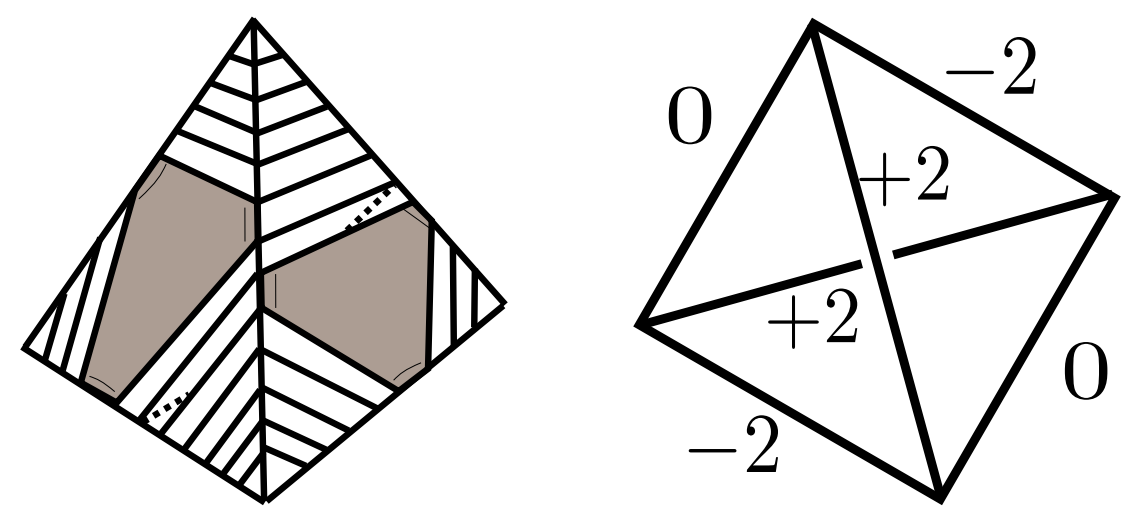

Figure 7. With two quads at the bottom edge of the left hexagon and top edge of the right hexagon, we have a shear of +2 along the edge between. The right figure gives all of the shearing coordinates on the tetrahedron. 
Now there is a unique way to glue together the arc patterns in two tetrahedra meeting along a common face, as the middle intervals in the faces must match up. The arc patterns from all the tetrahedra in the triangulation then fit together consistently (without any shearing) around an edge class if and only the sum of the shear parameters over all edges in the edge class vanishes. These conditions are precisely the Q-matching equations.

6.2. Geometric generators for Q-normal classes. The work of Kang-Rubinstein [KR04, Theorem 2.1] (also see Luo-Tilmann [LT08, §2]) shows that the space $\tilde{N}(\mathcal{T} ; \mathbb{R}) \subset \mathbb{R}^{7 n}$ of all closed normal classes satisfying Haken's matching equations (including, for example, the boundary tori) has a basis given by edge solutions $\tilde{E}_{i}$ and tetrahedron solutions $\tilde{T}_{j}$ where $1 \leq i, j \leq n$. These project to edge solutions $E_{i}$ and tetrahedron solutions $T_{j}$ under the map which forgets triangular coordinates, giving the image $N(\mathcal{T} ; \mathbb{R}) \subset \mathbb{R}^{3 n}$. Define $Q_{0}(\mathcal{T} ; \mathbb{R})$ as the solutions of the $Q$-matching equations corresponding to closed normal surfaces. It is a result of [Tol98] that the $Q$-coordinates determine a normal surface up to multiples of the peripheral normal tori and so $Q_{0}(\mathcal{T} ; \mathbb{R})=N(\mathcal{T} ; \mathbb{R})$.

When considering the map from all closed normal classes to $Q$-normal solution space, the closed normal surface solutions coming from the boundary tori map to the zero vector. Thus, such a map introduces one linear relation for each cusp, which in fact gives the only relations (see for example the proof of [KR04, Theorem 3.1]), so $\operatorname{dim} Q_{0}(\mathcal{T} ; \mathbb{R})=2 n-r$, Explicitly, the quad coordinates for $E_{i}$ are precisely the coefficients in the edge equation for the $i$ th edge in $\mathcal{T}$, and the quad coordinates for $T_{j}$ are $(1,1,1)$ in the $j$ th tetrahedron and $(0,0,0)$ in all other tetrahedra.

The space $Q(\mathcal{T} ; \mathbb{R})$ of all Q-normal classes is spanned by edge solutions, tetrahedron solutions and peripheral curve solutions (see [KR04, Theorem 3.1]). The peripheral curve solutions are linearly independent from the edge and tetrahedron solutions, and there are two linearly independent peripheral curve solutions $M_{k}, L_{k}$ per cusp corresponding to a choice of basis ("meridian" $\mu_{k}$, "longitude" $\left.\lambda_{k}\right)$ of $H_{1}\left(\partial_{k} M ; \mathbb{R}\right)$ for each component $\partial_{k} M$ of $\partial M$. In fact, a suitable choice of $n-r$ edge solutions together with the $n$ tetrahedral solutions and the $2 r$ peripheral solutions form a basis for the real solution space $Q(\mathcal{T} ; \mathbb{R})$, which therefore has dimension $2 n+r$ (which also follows from [KR04, Theorem 3.1]).

In fact the statements above follow from the symplectic relations of Neumann-Zagier (see [NZ85], [Neu92], [Cho06]). Let $A$ be the $n \times 3 n$ gluing equation matrix for $\mathcal{T}$, with rows given by $E_{1}, \ldots, E_{n}$. Then the $n \times 3 n$ matrix of $Q$-matching equations $B$ is given by $B=A C$ where $C$ is the $3 n \times 3 n$ block diagonal matrix made up of $n$ copies of

$$
\left[\begin{array}{ccc}
0 & 1 & -1 \\
-1 & 0 & 1 \\
1 & -1 & 0
\end{array}\right]
$$

(see Tillmann [Til08]). We can also regard $C$ as the linear map $C: \mathbb{R}^{3 n} \rightarrow \mathbb{R}^{3 n}$ given by $C\left(a_{1}, b_{1}, c_{1}, a_{2}, b_{2}, c_{2}, \ldots, a_{n}, b_{n}, c_{n}\right)=\left(-b_{1}+c_{1},-c_{1}+a_{1},-a_{1}+b_{1}, \ldots,-b_{n}+c_{n},-c_{n}+a_{n},-a_{n}+b_{n}\right)$.

Then the $Q$-matching equations have coefficients $C\left(E_{i}\right)$ for $i=1, \ldots, n$. (Compare Section 6.1 above.) 
Now the skew-symmetric pairing of Neumann-Zagier is given by

$$
\omega: \mathbb{R}^{3 n} \times \mathbb{R}^{3 n} \rightarrow \mathbb{R}, \quad \omega\left(x, x^{\prime}\right)=C x \cdot x^{\prime}
$$

where $\cdot$ denotes the dot product. Explicitly, given vectors

$$
x=\left(a_{1}, b_{1}, c_{1}, a_{2}, b_{2}, c_{2}, \ldots, a_{n}, b_{n}, c_{n}\right), x^{\prime}=\left(a_{1}^{\prime}, b_{1}^{\prime}, c_{1}^{\prime}, a_{2}^{\prime}, b_{2}^{\prime}, c_{2}^{\prime}, \ldots, a_{n}^{\prime}, b_{n}^{\prime}, c_{n}^{\prime}\right) \in\left(\mathbb{R}^{3}\right)^{n}
$$

we have

$$
\omega\left(x, x^{\prime}\right)=\sum_{j=1}^{n}\left(\left|\begin{array}{cc}
a_{j} & a_{j}^{\prime} \\
b_{j} & b_{j}^{\prime}
\end{array}\right|+\left|\begin{array}{cc}
b_{j} & b_{j}^{\prime} \\
c_{j} & c_{j}^{\prime}
\end{array}\right|+\left|\begin{array}{cc}
c_{j} & c_{j}^{\prime} \\
a_{j} & a_{j}^{\prime}
\end{array}\right|\right) .
$$

(Compare [Neu92, Cho06].)

It follows that $S \in \mathbb{R}^{3 n}$ satisfies the $Q$-matching equations if and only if

$$
\omega\left(E_{i}, S\right)=C\left(E_{i}\right) \cdot S=0 \text { for all } i=1, \ldots, n .
$$

It is immediate that each tetrahedral solution $T_{j}$ satisfies the $Q$-matching equations; in fact, $C\left(T_{j}\right)=0$ so $\omega\left(x, T_{j}\right)=-\omega\left(T_{j}, x\right)=-C\left(T_{j}\right) \cdot x=0$ for all $x \in \mathbb{R}^{3 n}$. Further, the Neumann-Zagier symplectic relations say that all symplectic products of $E_{i}, M_{k}, L_{k}$ for $i=1, \ldots, n, k=1, \ldots, r$ are zero except that

$$
\omega\left(L_{k}, M_{k}\right)=2=-\omega\left(M_{k}, L_{k}\right) \text { for each } k=1, \ldots, r .
$$

Hence, we can conclude that the edge solutions $E_{i}$ and peripheral solutions $M_{k}, L_{k}$ satisfy the $Q$-matching equations. In fact, with some more analysis, it can been seen that the tetrahedral solutions together with a suitable choice of $n-r$ edge solutions and the peripheral solutions form a basis for the real solution space $Q(\mathcal{T} ; \mathbb{R})$, which has dimension $2 n+r$.

As mentioned above, there is a basis for the $Q$-coordinates of spun normal surfaces given by appropriately chosen edge solutions $\left\{E_{i}\right\}_{i=1}^{n-r}$, all tetrahedral solutions $\left\{T_{j}\right\}_{j=1}^{n}$, and peripheral solutions $\left\{p_{k} M_{k}+q_{k} L_{k}\right\}_{k=1}^{r}$. Hence for each choice of $x_{i}, y_{j}, p_{k}, q_{k} \in \mathbb{R}$ we obtain a spun normal class

$$
S=\sum_{i} x_{i} E_{i}+\sum_{j} y_{i} T_{j}+\sum_{k}\left(p_{k} M_{k}+q_{k} L_{k}\right) .
$$

A normal surface has two important invariants; its Euler characteristic and its boundary slope. These invariants give rise to two linear maps on the normal surface solution space $Q(\mathcal{T} ; \mathbb{R})$.

Definition 6.1. The formal Euler characteristic is the linear map

$$
\chi: Q(\mathcal{T} ; \mathbb{R}) \rightarrow \mathbb{R}, \quad \chi(S)=\sum_{i}-2 x_{i}-\sum_{j} y_{j}
$$

giving the usual Euler characteristic for embedded closed and spun normal surfaces.

(See [LT08] for a detailed discussion of the closed case, and Appendix A for the spun normal case using generalised angle structures.)

Definition 6.2. The boundary map

$$
\partial: Q(\mathcal{T} ; \mathbb{R}) \rightarrow H_{1}(\partial M ; \mathbb{R}), \quad \partial(S)=2 \sum_{k}\left(p_{k} \mu_{k}+q_{k} \lambda_{k}\right)
$$


gives the boundary slope and the direction of spinning for embedded spun normal surfaces. Here $\mu_{k}$ and $\lambda_{k}$ are the meridian and longitude of cusp $k$ as defined previously.

(See Tillmann [Til08] for the details. ${ }^{1}$ ) Note that at each cusp, a tail of a spun normal surface is an infinite annulus spiralling into the cusp. There are two possible directions of spinning for each such a tail.

We can compute the boundary in terms of the Neumann-Zagier symplectic form: since

$$
\omega\left(S, M_{k}\right)=\omega\left(p_{k} M_{k}+q_{k} L_{k}, M_{k}\right)=-2 q_{k}
$$

and

$$
\omega\left(S, L_{k}\right)=\omega\left(p_{k} M_{k}+q_{k} L_{k}, L_{k}\right)=2 p_{k}
$$

we have

$$
\partial(S)=\sum_{k}\left(-\omega\left(S, L_{k}\right) \mu_{k}+\omega\left(S, M_{k}\right) \lambda_{k}\right)
$$

Note that

$$
\partial(S)=0 \text { if and only if } S \text { defines a closed normal class. }
$$

Hence $N(\mathcal{T} ; \mathbb{R})$ is the solution space of the $Q$-matching equations (17) together with the additional equations

$$
\omega\left(M_{k}, S\right)=\omega\left(L_{k}, S\right)=0 \text { for all } k=1, \ldots, r .
$$

We now define a quadratic function which will play an important role in understanding the degree of terms in the 3D-index sums.

Definition 6.3. We define a double arc function

$$
\delta: Q(\mathcal{T} ; \mathbb{R}) \rightarrow \mathbb{R}, \quad \delta(S)=\sum_{j}\left(a_{j} b_{j}+b_{j} c_{j}+c_{j} a_{j}\right),
$$

for

$$
S=\left(a_{1}, b_{1}, c_{1}, a_{2}, b_{2}, c_{2}, \ldots, a_{n}, b_{n}, c_{n}\right) .
$$

Note that for $S \in Q\left(\mathcal{T} ; \mathbb{Z}_{+}\right), a_{j} b_{j}+b_{j} c_{j}+c_{j} a_{j}$ counts the number of arcs of intersection between the quads of $S$ in tetrahedron $j$. In particular,

a normal surface solution $S \in Q\left(\mathcal{T} ; \mathbb{Z}_{+}\right)$is embedded if and only if $\delta(S)=0$.

There is also an associated symmetric bilinear function

$$
\delta: Q(\mathcal{T} ; \mathbb{R}) \times Q(\mathcal{T} ; \mathbb{R}) \rightarrow \mathbb{R}
$$

such that

$$
\delta\left(S+S^{\prime}\right)=\delta(S)+\delta\left(S^{\prime}\right)+2 \delta\left(S, S^{\prime}\right) \text { for all } S, S^{\prime} \in Q(\mathcal{T} ; \mathbb{R})
$$

Explicitly

$$
\delta\left(S, S^{\prime}\right)=\frac{1}{2} \sum_{j}\left(a_{j} b_{j}^{\prime}+b_{j} c_{j}^{\prime}+c_{j} a_{j}^{\prime}\right)+\left(a_{j}^{\prime} b_{j}+b_{j}^{\prime} c_{j}+c_{j}^{\prime} a_{j}\right)
$$

\footnotetext{
${ }^{1}$ Our sign convention is opposite to that in [Til08] and Regina v.4.96 ([Bur]), but is consistent with the boundary map defined in [Neu92] and discussed in $\S 7$ below.
} 
if $S$ and $S^{\prime}$ have quad coordinates in $\mathbb{R}^{3 n}$ given by

$$
[S]=\left(a_{1}, b_{1}, c_{1}, a_{2}, b_{2}, c_{2}, \ldots, a_{n}, b_{n}, c_{n}\right)
$$

and

$$
\left[S^{\prime}\right]=\left(a_{1}^{\prime}, b_{1}^{\prime}, c_{1}^{\prime}, a_{2}^{\prime}, b_{2}^{\prime}, c_{2}^{\prime}, \ldots, a_{n}^{\prime}, b_{n}^{\prime}, c_{n}^{\prime}\right) .
$$

\section{Combinatorics of ideal tRiangulations AND INTEGER NORMAL SURFACE THEORY}

The work of Kang-Rubinstein and Luo-Tillmann, described above, shows that the edge solutions and tetrahedral solutions span the real vector space $N(\mathcal{T} ; \mathbb{R}) \subset \mathbb{R}^{3 n}$ of closed normal classes, and that these together with the peripheral curve solutions span the real vector space $Q(\mathcal{T} ; \mathbb{R}) \subset \mathbb{R}^{3 n}$ of all $Q$-normal classes. However, over the integers the situation is more subtle - the integer linear combinations of edge and tetrahedral solutions generally give only a finite index submodule of $N(\mathcal{T} ; \mathbb{Z})$ and the integer linear combinations of edge, tetrahedral and peripheral curve solutions give a finite index submodule of $Q(\mathcal{T} ; \mathbb{Z})$. In this section we give a precise description of these integer classes. It turns out that this is a consequence of the results of Neumann [Neu92] on combinatorics of ideal triangulations.

We regard a $Q$-normal class as a linear combination of quads in $\mathcal{T}$ satisfying the $Q$-matching equations of Tollefson [Tol98]. Explicitly, let $\mathbb{Z} E \cong \mathbb{Z}^{n}$ and $\mathbb{Z} \square \cong \mathbb{Z}^{3 n}$ denote the free $\mathbb{Z}_{\text {- }}$ modules with bases given by the (unoriented) edge classes and quad types in $\mathcal{T}$ respectively. Given a quad $q_{\sigma}$ in an oriented tetrahedron $\sigma$ of $\mathcal{T}$ we associate a sign \pm 1 to each of the edges of $\sigma$ meeting $q_{\sigma}$ as shown in figure 8. (These signs give the shear parameters along the edges as explained in Section 6.1.) Adding these contributions gives a linear map

$$
F: \mathbb{Z} \square \rightarrow \mathbb{Z} E
$$

whose kernel is the submodule $Q(\mathcal{T} ; \mathbb{Z})$ of $Q$-normal classes.
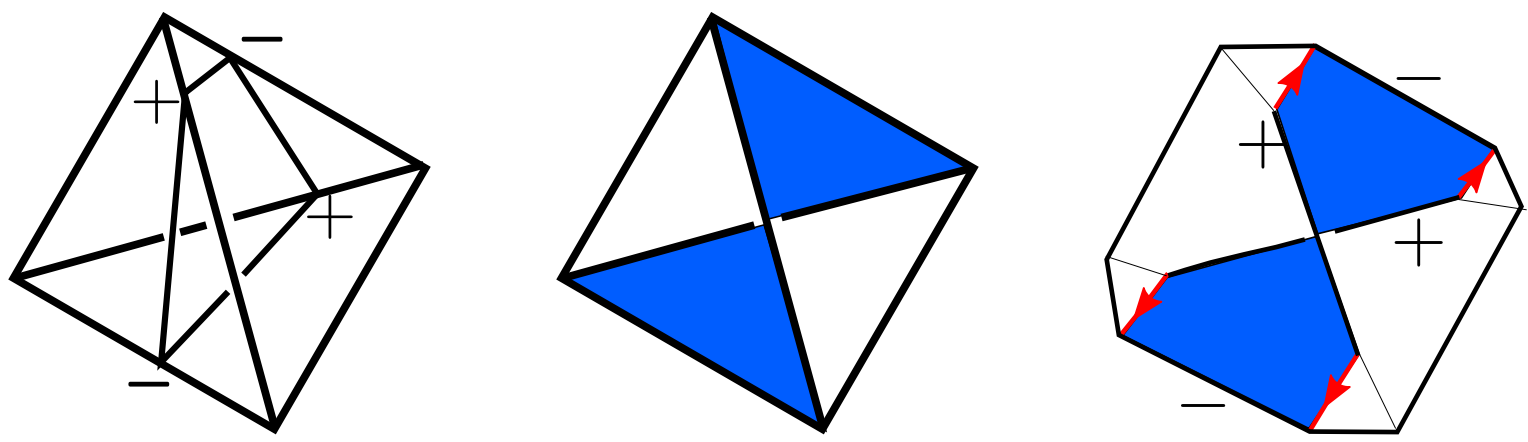

FIGURE 8. Each quad can be replaced by a twisted square or twisted octagon.

Kang-Rubinstein [KR04] observed that for each edge class in $\mathcal{T}$, the sum of all quad types facing the edges in this class gives an "edge solution" in $Q(\mathcal{T} ; \mathbb{Z})$; this gives a linear map

$$
G: \mathbb{Z} E \rightarrow \mathbb{Z} \square
$$

with image contained in $Q(\mathcal{T} ; \mathbb{Z})$. Further, the sum of the three quad types in a tetrahedron in $\mathcal{T}$ gives a "tetrahedral solution" in $Q(\mathcal{T} ; \mathbb{Z})$. We let $\mathbb{E}, \mathbb{T} \subset Q(\mathcal{T} ; \mathbb{Z}) \subset \mathbb{Z} \square$ denote the 
$\mathbb{Z}$-submodules spanned by the edge solutions and tetrahedral solutions respectively, and let $Q_{0}(\mathcal{T} ; \mathbb{Z})$ denote the submodule $\mathbb{E}+\mathbb{T} \subset Q(\mathcal{T} ; \mathbb{Z})$.

Next we associate two important homology (or cohomology) classes with any $Q$-normal class. First observe that any quadrilateral in a tetrahedron can be replaced by a 'twisted square' meeting the same edges, or by a 'twisted octagon' in the corresponding truncated tetrahedron with its four external edges oriented as shown in Figure 8 (compare [Neu92, Figures 9, 10]). Note that the arrow on each external edge goes from an internal edge labelled +1 to an internal edge labelled -1 .

Each spun normal class $S \in Q(\mathcal{T} ; \mathbb{Z})$ is a linear combination of quads, so we can replace this by a linear combination $S^{\prime}$ of twisted octagons. It follows from the $Q$-matching equations that $S^{\prime}$ represents a mod 2 homology class $[S]_{2} \in H_{2}(M, \partial M ; \mathbb{Z} / 2 \mathbb{Z})$. Here and throughout the paper, we will use []$_{2}$ to indicate a $\mathbb{Z} / 2 \mathbb{Z}$ homology (or co-homology) class. Further, the boundary edges of $S^{\prime}$, oriented as above, represent a homology class $[\partial S] \in H_{1}(M ; \mathbb{Z})$. In particular, the kernel of the boundary map $S \mapsto[\partial S]$ is precisely the submodule $N(\mathcal{T} ; \mathbb{Z}) \subset$ $Q(\mathcal{T} ; \mathbb{Z})$ of closed normal classes.

When $S$ gives an embedded spun normal surface, $[\partial S]$ agrees with boundary map defined by Tillmann in [Til08] (up to sign), and describes the boundary components of the spun normal surface and the direction of spiralling of the spun normal surface around $\partial M$. (With the orientation convention given in Figure 8, the ends of a spun normal surface spiral up into the cusp to the right of the oriented boundary curve, as viewed from the cusp.)

Since $S^{\prime}$ gives a 2 -chain mod 2 whose boundary represents the reduction of $[\partial S] \bmod 2$, it follows that

$$
\left([S]_{2},[\partial S]\right) \in\left\{(a, b) \in H_{2}(M, \partial M ; \mathbb{Z} / 2 \mathbb{Z}) \times H_{1}(\partial M ; \mathbb{Z}): \partial_{*} a=b \bmod 2\right\}
$$

where $\partial_{*}: H_{2}(M, \partial M ; \mathbb{Z} / 2 \mathbb{Z}) \rightarrow H_{1}(\partial M ; \mathbb{Z} / 2 \mathbb{Z})$ is the connecting homomorphism in the long exact sequence of the pair $(M, \partial M)$. Hence, $[\partial S] \in \mathcal{K}=\operatorname{Ker}\left(H_{1}(\partial M ; \mathbb{Z}) \rightarrow H_{1}(M ; \mathbb{Z} / 2 \mathbb{Z})\right)$, by the long exact sequence of the pair $(M, \partial M)$ with $\mathbb{Z} / 2 \mathbb{Z}$ coefficients.

Theorem 7.1. The homomorphism

$$
H: Q(\mathcal{T} ; \mathbb{Z}) \rightarrow H_{2}(M, \partial M ; \mathbb{Z} / 2 \mathbb{Z}) \times H_{1}(\partial M ; \mathbb{Z}), S \mapsto\left([S]_{2},[\partial S]\right)
$$

has image

$$
\operatorname{Im} H=\left\{(a, b) \in H_{2}(M, \partial M ; \mathbb{Z} / 2 \mathbb{Z}) \times H_{1}(\partial M ; \mathbb{Z}): \partial_{*} a=b \bmod 2\right\}
$$

and kernel

$$
\operatorname{Ker} H=Q_{0}(\mathcal{T} ; \mathbb{Z})=\mathbb{E}+\mathbb{T} .
$$

In particular, the homomorphism

$$
H_{0}: N(\mathcal{T} ; \mathbb{Z}) \rightarrow H_{2}(M, \partial M ; \mathbb{Z} / 2 \mathbb{Z}), S \mapsto[S]_{2}
$$

has image

$\operatorname{Im} H_{0}=\operatorname{Ker}\left(H_{2}(M, \partial M ; \mathbb{Z} / 2 \mathbb{Z}) \rightarrow H_{2}(\partial M ; \mathbb{Z} / 2 \mathbb{Z})\right)=\operatorname{Im}\left(H_{2}(M ; \mathbb{Z} / 2 \mathbb{Z}) \rightarrow H_{2}(M, \partial M ; \mathbb{Z} / 2 \mathbb{Z})\right)$ and kernel

$\operatorname{Ker} H_{0}=Q_{0}(\mathcal{T} ; \mathbb{Z})=\mathbb{E}+\mathbb{T}$ 
Proof. This essentially follows from Neumann [Neu92, Theorem 5.1] together with Poincaré duality. To match Neumann's notation from sections 4-6 of [Neu92] we let $K$ denote the oriented pseudo-manifold given by the triangulation $\mathcal{T}$ with its ideal vertices included. Thus $K$ is homeomorphic to the end compactification $\hat{M}$ of the $\operatorname{int} M$, obtained by collapsing each component of $\partial M$ to a separate point. Truncating the corners of the tetrahedra in $K$ gives a cell complex $K_{0}$ homeomorphic to $M$ with boundary $\partial K_{0}$ homeomorphic to $\partial M$.

Let $C_{0}=\mathbb{Z} V \cong \mathbb{Z}^{r}, C_{1}=\mathbb{Z} E \cong \mathbb{Z}^{n}, \bar{J}=\mathbb{Z} \square \cong \mathbb{Z}^{3 n}$ be the free $\mathbb{Z}$-modules with bases given by the cusps, (unoriented) edge classes, and quad types in $\mathcal{T}$ respectively, and let $\mathbb{E}, \mathbb{T} \subset \mathbb{Z} \square$ denote the $\mathbb{Z}$-submodules spanned by the edge solutions and tetrahedral solutions respectively. Then Neumann defines a chain complex

$$
0 \rightarrow C_{0} \stackrel{\alpha}{\rightarrow} C_{1} \stackrel{\beta}{\rightarrow} J \stackrel{\beta^{*}}{\rightarrow} C_{1} \stackrel{\alpha^{*}}{\rightarrow} C_{0} \rightarrow 0
$$

where $J=\mathbb{Z} \square / \mathbb{T} \cong \mathbb{Z}^{2 n}, \alpha$ gives the sum of all edges incident to a vertex, $\alpha^{*}$ is a map that associates to an edge the sum of its endpoints, and $\beta$ and $\beta^{*}$ are defined so that the following diagram commutes:

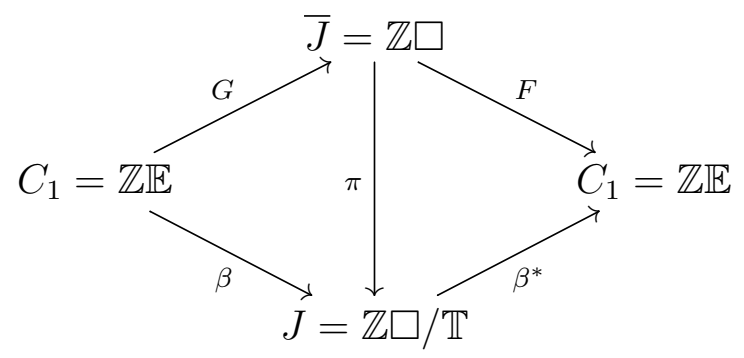

Here, $\pi: \mathbb{Z} \square \rightarrow \mathbb{Z} \square / \mathbb{T}$ is the quotient map.

To relate this to integer $Q$-normal surface theory we observe that $\operatorname{Ker} \beta^{*}=Q(\mathcal{T} ; \mathbb{Z}) / \mathbb{T}$ and $\operatorname{Im} \beta=(\mathbb{E}+\mathbb{T}) / \mathbb{T}$.

Now Neumann's Theorem 5.1 gives an isomorphism from $\operatorname{Ker} \beta^{*} / \operatorname{Im} \beta=Q(\mathcal{T} ; \mathbb{Z}) /(\mathbb{E}+\mathbb{T})$ to

$$
\left\{(f, g) \in H^{1}(M ; \mathbb{Z} / 2 \mathbb{Z}) \times H^{1}(\partial M ; \mathbb{Z}): i^{*} f=g \bmod 2\right\}
$$

where $i^{*}: H^{1}(M ; \mathbb{Z} / 2 \mathbb{Z}) \rightarrow H^{1}(\partial M ; \mathbb{Z} / 2 \mathbb{Z})$ is induced by the inclusion map $i: \partial M \rightarrow$ $M$. In other words, this is the set of pairs $(f, g)$ with $f \in \operatorname{Hom}\left(H_{1}(M), \mathbb{Z} / 2 \mathbb{Z}\right), g \in$ $\operatorname{Hom}\left(H_{1}(\partial M), \mathbb{Z}\right)$, such that $f(\gamma)=g(\gamma) \bmod 2$ for all $\gamma \in H_{1}(\partial M)$, where we write $H_{1}(\partial M)=H_{1}(\partial M ; \mathbb{Z})$ and $H_{1}(M)=H_{1}(M ; \mathbb{Z})$.

Now we have a commutative diagram

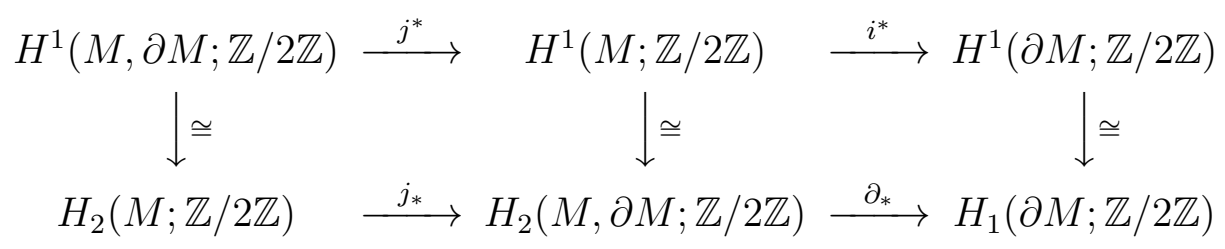

where the vertical maps are the isomorphisms given by Poincaré duality. This gives an isomorphism

$$
Q(\mathcal{T} ; \mathbb{Z}) /(\mathbb{E}+\mathbb{T}) \cong\left\{(a, b) \in H_{2}(M, \partial M ; \mathbb{Z} / 2 \mathbb{Z}) \times H_{1}(\partial M ; \mathbb{Z}): \partial_{*} a=b \bmod 2\right\}
$$


where $\partial_{*}: H_{2}(M, \partial M ; \mathbb{Z} / 2 \mathbb{Z}) \rightarrow H_{1}(\partial M ; \mathbb{Z} / 2 \mathbb{Z})$ is the connecting homomorphism in the long exact sequence of the pair $(M, \partial M)$. A careful examination of Neumann's work shows that this isomorphism is given by the homology map $H: S \mapsto\left([S]_{2},[\partial S]\right)$ defined above.

Remark 7.2. Using Poincaré duality and the Mayer-Vietoris sequence it follows that

$$
\begin{aligned}
\operatorname{Im} H_{0} & =\operatorname{Im}\left(H_{2}(M ; \mathbb{Z} / 2 \mathbb{Z}) \rightarrow H_{2}(M, \partial M ; \mathbb{Z} / 2 \mathbb{Z})\right) \\
& \cong \operatorname{Ker}\left(H^{1}(M ; \mathbb{Z} / 2 \mathbb{Z}) \rightarrow H^{1}(\partial M ; \mathbb{Z} / 2 \mathbb{Z})\right) \cong H^{1}(\hat{M} ; \mathbb{Z} / 2 \mathbb{Z}) .
\end{aligned}
$$

where $\hat{M}$ is the pseudo-manifold given by the triangulation $\mathcal{T}$ with its ideal vertices included; thus $\hat{M}$ is homeomorphic to $M$ with a cone attached over each boundary torus.

Remark 7.3. When $M$ has no non-peripheral $\mathbb{Z} / 2 \mathbb{Z}$ homology (for example, if $M$ is a knot or link exterior in a $\mathbb{Z} / 2 \mathbb{Z}$ homology sphere), then $Q_{0}(\mathcal{T} ; \mathbb{Z})=N(\mathcal{T} ; Z)$ consists of all closed normal classes. Further, $\mathcal{K}=\operatorname{Ker}\left(H_{1}(\partial M ; \mathbb{Z}) \rightarrow H_{1}(M ; \mathbb{Z} / 2 \mathbb{Z})\right)$ is a subgroup of index $2^{r}$ in $H_{1}(\partial M ; \mathbb{Z})$ where $r$ is the number of components of $\partial M$.

In general, $Q_{0}(\mathcal{T} ; \mathbb{Z})$ is a finite index submodule of $N(\mathcal{T} ; Z)$, and for any normal surface class $S \in N(\mathcal{T} ; Z)$ its "double" $2 S$ lies in $Q_{0}(\mathcal{T} ; \mathbb{Z})$.

7.1. Geometric generators for integer Q-normal classes. Next we describe explicit generators for $N(\mathcal{T} ; \mathbb{Z})$. First, recall that $Q_{0}(\mathcal{T} ; \mathbb{Z})=\mathbb{E}+\mathbb{T}$ is generated by the edge solutions and tetrahedral solutions described by Kang-Rubinstein [KR04]. It follows from Theorem 7.1 and the previous remark that

$$
N(\mathcal{T} ; \mathbb{Z}) / Q_{0}(\mathcal{T} ; \mathbb{Z}) \cong \operatorname{Im} H_{0} \cong H^{1}(\hat{M} ; \mathbb{Z} / 2 \mathbb{Z}) .
$$

Given a $\bmod 2$ class $a \in \operatorname{Im} H_{0}=\operatorname{Im}\left(H_{2}(M ; \mathbb{Z} / 2 \mathbb{Z}) \rightarrow H_{2}(M, \partial M ; \mathbb{Z} / 2 \mathbb{Z})\right)$, we can construct a closed normal class $S_{0} \in N(\mathcal{T} ; \mathbb{Z})$ with $\left[S_{0}\right]_{2}=a$ and $\left[\partial S_{0}\right]=0$ as follows. Choose a simplicial 1-cocycle $z \in Z^{1}(\mathcal{T} ; \mathbb{Z} / 2 \mathbb{Z})$ representing the dual cohomology class in $H^{1}(\hat{M} ; \mathbb{Z} / 2 \mathbb{Z})$. This assigns an element $\overline{0}$ or $\overline{1} \in \mathbb{Z} / 2 \mathbb{Z}$ to each edge of $\mathcal{T}$, and we lift these to integers $0,1 \in \mathbb{Z}$ giving a 1 -cochain $\tilde{z} \in C^{1}(\mathcal{T} ; \mathbb{Z})$. Since $z$ is a $\bmod 2$ cocycle, it follows that the sum of integers attached to the edges of any triangle in $\mathcal{T}$ is even. This implies that the integers attached to edges of any tetrahedra in $\mathcal{T}$ are either: (i) all 0 , (ii) 1 at the three edges of a triangular normal disc, 0 elsewhere (ii) or 1 at the four edges of a quad, 0 elsewhere. Now we can construct an embedded closed normal surface $S$ by taking one triangle in each tetrahedron of type (ii) and one quad in tetrahedron of type (iii). Forgetting the triangle coordinates (if any) gives a closed normal class $S_{0}$ in $N(\mathcal{T} ; \mathbb{Z})$ such that $\left[S_{0}\right]_{2}=a$ and $\left[\partial S_{0}\right]=0$.

Remark 7.4. Changing $z$ by a coboundary changes $S$ by adding vertex linking surfaces. But there is a unique closed embedded normal surface of least weight (i.e. having fewest intersections with the edges) obtained by this construction.

Given any class $b \in H_{1}(\partial M ; \mathbb{Z})$, a construction of Neumann [Neu92] gives a normal class $S \in Q(\mathcal{T} ; \mathbb{Z})$ with $[\partial S]=2 b$ as follows. Represent $b$ by an oriented multi-curve $\beta$ which is in normal position relative to the induced triangulation $\mathcal{T}_{\partial}$ of $\partial M$. Each oriented normal arc of $\beta$ lies in a truncated tetrahedron $\sigma$ of $\mathcal{T}$ and winds around one of the edges of $\sigma$, which faces a quad type $q$ in $\sigma$. Now add up the quads associated to all the normal arcs of $\beta$, with signs 
+1 or -1 according to whether the normal arc winds anticlockwise or clockwise as viewed from the cusp. The result is a normal class with $[\partial S]=2 b,[S]_{2}=0$ and $\chi(S)=0$.

Remark 7.5. These "peripheral curve solutions" give normal classes in $Q(\mathcal{T} ; \mathbb{Z})$ representing all even classes $b \in H_{1}(\partial M ; 2 \mathbb{Z}) \subset H_{1}(\partial M ; \mathbb{Z})$, i.e. those such that $b \bmod 2=0$. Adding these to the above closed normal classes gives normal classes representing all $(a, b) \in$ $H_{2}(M, \partial M ; \mathbb{Z} / 2 \mathbb{Z}) \times H_{1}(\partial M ; \mathbb{Z})$ such that $\partial_{*} a=b \bmod 2=0$. We do not know a direct geometric construction for the other (non-even) classes $b \in \mathcal{K} \subset H_{1}(\partial M ; \mathbb{Z})$.

Remark 7.6. For any $Q$-normal class $S \in Q(\mathcal{T} ; \mathbb{Z})$, its double satisfies $[\partial(2 S)] \in H_{1}(\partial M ; 2 \mathbb{Z})$ and $[2 S]_{2}=0 \in H_{2}(M, \partial M ; \mathbb{Z} / 2 \mathbb{Z})$. Hence $2 S$ is an integer linear combination of edge solutions, tetrahedral solutions and peripheral curve solutions.

\section{The 3D-INDEX VIA NORMAL SURFACES}

We first extend the tetrahedral index $J_{\Delta}$ to a function $J: \mathbb{Z}^{3 n} \rightarrow \mathbb{Z}\left(\left(q^{1 / 2}\right)\right)$ by defining

$$
J\left(a_{1}, b_{1}, c_{1}, a_{2}, b_{2}, c_{2}, \ldots, a_{n}, b_{n}, c_{n}\right)=\prod_{j=1}^{n} J_{\Delta}\left(a_{j}, b_{j}, c_{j}\right),
$$

and for each $Q$-normal class $S \in Q(\mathcal{T} ; \mathbb{Z})$ we define

$$
I(S)=\left(-q^{1 / 2}\right)^{-\chi(S)} J(S),
$$

where $\chi$ is the formal Euler characteristic. Then $I(S)$ is unchanged by adding tetrahedral solutions: if $S^{*}=S+\sum_{j} m_{j} T_{j}$ with $m_{j} \in \mathbb{Z}$ then $J(S)=\left(-q^{1 / 2}\right)^{\sum_{j} m_{j}} J\left(S^{*}\right)$ and $-\chi(S)=$ $-\chi\left(S^{*}\right)-\sum_{j} m_{j}$, so $I(S)=I\left(S^{*}\right)$. So there is a well-defined function on the quotient group

$$
I: Q(\mathcal{T} ; \mathbb{Z}) / \mathbb{T} \rightarrow \mathbb{Z}\left(\left(q^{1 / 2}\right)\right),
$$

where $\mathbb{T}=\sum_{j} \mathbb{Z} T_{j} \subset Q(\mathcal{T} ; \mathbb{Z})$ is the subgroup generated by the tetrahedral solutions.

Taking homology classes of $Q$-normal classes gives a function $S \mapsto\left([S]_{2},[\partial S]\right)$ which also vanishes on $\mathbb{T}$, so gives a well-defined homomorphism

$$
h: Q(\mathcal{T} ; \mathbb{Z}) / \mathbb{T} \rightarrow H_{2}(M, \partial M ; \mathbb{Z} / 2 \mathbb{Z}) \times H_{1}(\partial M ; \mathbb{Z}),
$$

and Theorem 7.1 shows that

$$
\operatorname{Im} h=\left\{(a, b) \in H_{2}(M, \partial M ; \mathbb{Z} / 2 \mathbb{Z}) \times H_{1}(\partial M ; \mathbb{Z}): \partial_{*} a=b \bmod 2\right\}
$$

and

$$
\operatorname{Ker} h=Q_{0}(\mathcal{T} ; \mathbb{Z}) / \mathbb{T}=(\mathbb{E}+\mathbb{T}) / \mathbb{T}
$$

where $\mathbb{E}=\sum_{i} \mathbb{Z} E_{i} \subset Q(\mathcal{T} ; \mathbb{Z})$ is the subgroup generated by the edge solutions.

Definition 8.1. For each $(a, b) \in \operatorname{Im} h$ we define an extended version of the 3D-index by

$$
I_{\mathcal{T}}^{a}(b)=\sum_{[S] \in h^{-1}(a, b)} I([S]) .
$$


To compute this, we can choose a normal class $S_{0} \in Q(\mathcal{T} ; \mathbb{Z})$ with $\left[S_{0}\right]_{2}=a,\left[\partial S_{0}\right]=b$ and choose a set of $n-r$ edge solutions (as explained in [GHRS15]), say $E_{1}, \ldots, E_{n-r}$, whose integer linear combinations form a complete set of coset representatives for $(\mathbb{E}+\mathbb{T}) / \mathbb{T}$. Then

$$
I_{\mathcal{T}}^{a}(b)=\sum_{\boldsymbol{k}=\left(k_{1}, \ldots, k_{n-r}\right) \in \mathbb{Z}^{n-r}} I\left(S_{0}+\sum_{i} k_{i} E_{i}\right) .
$$

Corollary 8.2. In particular, the index $I_{\mathcal{T}}^{0}(0)$ is a sum over closed normal classes in $Q_{0}(\mathcal{T} ; \mathbb{Z})$ modulo tetrahedral solutions:

$$
I_{\mathcal{T}}^{0}(0)=\sum_{[Q] \in Q_{0}(\mathcal{T} ; \mathbb{Z}) / \mathbb{T}} I([S])=\sum_{\boldsymbol{k}=\left(k_{1}, \ldots, k_{n-r}\right) \in \mathbb{Z}^{n-r}} I\left(\sum_{i} k_{i} E_{i}\right) .
$$

Remark 8.3. It follows immediately from the definition that $I_{\mathcal{T}}^{a}(-b)=I_{\mathcal{T}}^{a}(b)$ for all $a, b$.

Remark 8.4. Note on Notation. The previous definition of 3D-index from [GHRS15] only applies to the cases where $a=0$ and $b \in H_{1}(\partial M ; 2 \mathbb{Z})$. We then have $I_{\mathcal{T}}^{0}(b)=I_{\mathcal{T}}(b / 2)$ in the notation of [GHRS15]. For the complement $M$ of a knot in $S^{3}$ with standard meridian $\mu$ and longitude $\lambda$, the index $I_{\mathcal{T}}(m, e)$ in [DGG13] is denoted $I_{\mathcal{T}}(e \mu-m \lambda / 2)$ in [GHRS15] and $I_{\mathcal{T}}^{0}(2 e \mu-m \lambda)$ here.

8.1. Convergence of the index sum. To understand the convergence of the index sum (as a formal Laurent series) we need to examine the lowest degree of the terms in this sum. Given any coset $[S]=S+\mathbb{T}$, we can choose its "minimal non-negative coset representative" $S^{*}$. Explicitly, given

$$
S=\left(a_{1}, b_{1}, c_{1}, a_{2}, b_{2}, c_{2}, \ldots, a_{n}, b_{n}, c_{n}\right) \in Q(\mathcal{T} ; \mathbb{Z})
$$

we have

$$
S^{*}=\left(a_{1}^{*}, b_{1}^{*}, c_{1}^{*}, a_{2}^{*}, b_{2}^{*}, c_{2}^{*}, \ldots, a_{n}^{*}, b_{n}^{*}, c_{n}^{*}\right),
$$

where $\left(a_{j}^{*}, b_{j}^{*}, c_{j}^{*}\right)=\left(a_{j}-m_{j}, b_{j}-m_{j}, c_{j}-m_{j}\right)$ and $m_{j}=\min \left\{a_{j}, b_{j}, c_{j}\right\}$.

Now $J\left(S^{*}\right)$ has leading term $q^{\frac{1}{2} \delta\left(S^{*}\right)}$ of degree $\frac{1}{2} \delta\left(S^{*}\right)$. So each surface $[S]$ occurring in the index sum $(29)$ contributes a term $I([S])=\left(-q^{1 / 2}\right)^{-\chi\left(S^{*}\right)} J\left(S^{*}\right)$ of lowest $q^{1 / 2}$-degree

$$
d([S])=d\left(S^{*}\right)=-\chi\left(S^{*}\right)+\delta\left(S^{*}\right)
$$

and leading coefficient $(-1)^{-\chi\left(S^{*}\right)}$.

Remark 8.5. Note that $S^{*}$ need not give an embedded normal surface, but there are at most two non-zero quad coordinates in each tetrahedron. In the next section we will show how to replace such a normal class by a unique embedded generalised normal surface $\tilde{S}$.

From this, it is easy to analyse the convergence of the index sums. First we give a new proof that the sum $I_{\mathcal{T}}^{0}(0)$ converges (as formal Laurent series) if and only if $\mathcal{T}$ contains no embedded normal surfaces of Euler characteristic $\geq 0$.

Theorem 8.6. The index sum for $I_{\mathcal{T}}^{0}(0)$ converges (as a formal Laurent series) if and only if the triangulation $\mathcal{T}$ is 1-efficient. 
Proof. $(\Rightarrow)$ If the triangulation $\mathcal{T}$ is not 1-efficient then it contains a closed embedded normal surface with non-negative Euler characteristic. Doubling this, if necessary, gives a closed embedded normal surface $S \in Q_{0}\left(\mathcal{T} ; \mathbb{Z}_{+}\right)$with $\chi(S) \geq 0, \delta(S)=0$ and $S^{*}=S \neq 0$. Then for each integer $k \geq 0,(k S)^{*}=k S$ contributes a term to the sum (31) for $I_{\mathcal{T}}^{0}(0)$ with $q^{1 / 2}$-degree $d(k S)=-k \chi(S) \leq 0$. So the index sum cannot converge as a formal Laurent series in $q^{1 / 2}$.

$(\Leftarrow)$ Assume that $\mathcal{T}$ is a 1 -efficient ideal triangulation. First we note the following general fact.

Lemma 8.7. The function $d=-\chi+\delta: Q\left(\mathcal{T} ; \mathbb{R}_{+}\right) \rightarrow \mathbb{Z}$ is superadditive, i.e.

$$
d\left(S+S^{\prime}\right) \geq d(S)+d\left(S^{\prime}\right) .
$$

Hence $d\left(\sum_{i} n_{i} S_{i}\right) \geq \sum_{i} d\left(n_{i} S_{i}\right) \geq \sum_{i} n_{i} d\left(S_{i}\right)$ for all $S_{i} \in Q\left(\mathcal{T} ; \mathbb{R}_{+}\right)$and all integers $n_{i} \geq 0$.

Proof. This follows since $\chi$ is a linear function and $\delta\left(S+S^{\prime}\right) \geq \delta(S)+\delta\left(S^{\prime}\right)$ provided all quad coordinates of $S$ and $S^{\prime}$ are non-negative.

Next we observe that $N\left(\mathcal{T} ; \mathbb{Z}_{+}\right)$is the set of integer points in a cone defined by homogeneous linear inequalities with integer coefficients. So we can choose a set of fundamental solutions (or Hilbert basis in integer linear programming theory) $F_{1}, \ldots, F_{m}$ for $N\left(\mathcal{T} ; \mathbb{Z}_{+}\right)$. This means that every $S \in N\left(\mathcal{T} ; \mathbb{Z}_{+}\right)$can written (not necessarily uniquely) as a finite $\operatorname{sum} S=\sum_{i=1}^{m} n_{i} F_{i}$ where $n_{i} \in \mathbb{Z}_{+}$and each $F_{i}$ is irreducible, i.e. has no decomposition $F_{i}=x+y$ where $x, y \in N\left(\mathcal{T} ; \mathbb{Z}_{+}\right)$and $x, y \neq 0$.

Now consider $f_{i}(x)=d\left(x F_{i}\right)=-\chi\left(F_{i}\right) x+\delta\left(F_{i}\right) x^{2}$, where $x \geq 0$. Since $\mathcal{T}$ is 1-efficient, for each $i=1, \ldots, m$, we have either (i) $\delta\left(F_{i}\right)=0$ and $-\chi\left(F_{i}\right) \geq 1$, or (ii) $\delta\left(F_{i}\right) \geq 1$. Hence $f_{i}(x) \rightarrow \infty$ as $x \rightarrow+\infty$, and in fact there are constants $a_{i}, b_{i} \in \mathbb{R}$ with $a_{i}>0$ such that $f_{i}(x) \geq a_{i} x-b_{i}$ for all $x \geq 0$.

Then each $S \in N(\mathcal{T} ; \mathbb{Z})$ can be written as a linear combination $S=\sum_{i=1}^{m} x_{i} F_{i}$ with $x_{i} \in \mathbb{Z}_{+}$ and, using Lemma 8.7, its $q^{1 / 2}$-degree satisfies

$$
d(S) \geq \sum_{i=1}^{m} d\left(x_{i} F_{i}\right) \geq \sum_{i}\left(a_{i} x_{i}-b_{i}\right) .
$$

Thus, $d(S) \leq D$ implies $\sum_{i} a_{i} x_{i} \leq D+\sum_{i} b_{i}$, which has only finitely many solutions with $x_{i} \in \mathbb{Z}_{+}$. Hence $I_{\mathcal{T}}^{0}(0)$ converges as a formal Laurent series.

A similar argument gives the following

Theorem 8.8. If $\mathcal{T}$ is 1-efficient, then $I_{\mathcal{T}}^{a}(b)$ converges for all $a$ and $b$; in fact $\sum_{a} I_{\mathcal{T}}^{a}(b)$ is convergent for all $b$.

Proof. We may assume that $b \neq 0$. Now consider the set $Q_{b}\left(\mathcal{T} ; \mathbb{Z}_{+}\right)$of Q-normal classes $S \in Q\left(\mathcal{T} ; \mathbb{Z}_{+}\right)$whose boundary $\partial S$ is a non-negative integer multiple of $b$. This is the set of integer points in a cone defined by a set of homogeneous linear equations and inequalities with integer coefficients, so we can choose a finite set $F_{1}, \ldots, F_{m}$ of fundamental solutions for $Q_{b}\left(\mathcal{T} ; \mathbb{Z}_{+}\right)$where $\partial F_{i}=s_{i} b$ with $s_{i} \in \mathbb{Z}_{+}$.

Given $S \in Q_{b}\left(\mathcal{T} ; \mathbb{Z}_{+}\right)$we can write $S=\sum x_{i} F_{i}$ where $x_{i} \in \mathbb{Z}_{+}$and $\partial S=\left(\sum s_{i} x_{i}\right) b$. Let $I:=\{1,2, \ldots, m\}$ and write $I=I_{0} \cup I_{1}$ where $I_{0}=\left\{i \in I: s_{i}=0\right\}$ and $I_{1}=\left\{i \in I: s_{i} \geq 1\right\}$. 
Then $S=S_{0}+S_{1}$ where $S_{0}=\sum_{i \in I_{0}} x_{i} F_{i}$ and $S_{1}=\sum_{i \in I_{1}} x_{i} F_{i}$ and $\partial S=b$ if and only if $\sum_{i \in I_{1}} s_{i} x_{i}=1$. So $S_{1}$ belongs to the finite set $\left\{F_{i}: i \in I_{1}\right.$ and $\left.s_{i}=1\right\}$, and $S_{0} \in N(\mathcal{T} ; \mathbb{Z})$.

As in the proof of Theorem 8.6 there are constants $a_{i}, b_{i} \in \mathbb{R}$ with $a_{i}>0$ such that $d\left(x_{i} F_{i}\right) \geq a_{i} x_{i}-b_{i}$ for each $i \in I_{1}$. Now

$$
d(S) \geq d\left(S_{1}\right)+d\left(S_{0}\right) \geq d\left(S_{1}\right)+\sum_{i \in I_{1}} d\left(x_{i} F_{i}\right) \geq d\left(S_{1}\right)+\sum_{i \in I_{1}}\left(a_{i} x_{i}-b_{i}\right) .
$$

Hence given any $D \geq 0$ there are at most finitely many $S \in Q_{b}\left(\mathcal{T} ; \mathbb{Z}_{+}\right)$with $\partial S=b$ and $d(S) \leq D$. This implies the result.

Theorem 8.9. If $\mathcal{T}$ is spun 1-efficient, i.e. contains no embedded spun normal surface $S \neq 0$ with $\chi(S) \geq 0$, then the "total index" $I_{\mathcal{T}}^{\text {tot }}=\sum_{\text {all }(a, b)} I_{\mathcal{T}}^{a}(b)$ converges.

Proof. Let $F_{1}, \ldots, F_{m}$ be a set of fundamental solutions for the set $Q\left(\mathcal{T} ; \mathbb{Z}_{+}\right)$of non-negative integer solutions to the Q-matching equations.

Now consider $f_{i}(x)=d\left(x F_{i}\right)=-\chi\left(F_{i}\right) x+\delta\left(F_{i}\right) x^{2}$, where $x \geq 0$. Since $\mathcal{T}$ is spun 1-efficient, for each $i=1, \ldots, m$, we have either (i) $\delta\left(F_{i}\right)=0$ and $-\chi\left(F_{i}\right) \geq 1$, or (ii) $\delta\left(F_{i}\right) \geq 1$. Hence $f_{i}(x) \rightarrow \infty$ as $x \rightarrow+\infty$, and in fact there are constants $a_{i}, b_{i} \in \mathbb{R}$ with $a_{i}>0$ such that $f_{i}(x) \geq a_{i} x-b_{i}$ for all $x \geq 0$.

Then each $S \in Q(\mathcal{T} ; \mathbb{Z})$ can be written as a linear combination $S=\sum_{i=1}^{m} x_{i} F_{i}$ with $x_{i} \in \mathbb{Z}_{+}$ and, using Lemma 8.7, its $q^{1 / 2}$-degree satisfies

$$
d(S) \geq \sum_{i=1}^{m} d\left(x_{i} F_{i}\right) \geq \sum_{i}\left(a_{i} x_{i}-b_{i}\right) .
$$

Thus, $d(S) \leq D$ implies $\sum_{i} a_{i} x_{i} \leq D+\sum_{i} b_{i}$, which has only finitely many solutions with $x_{i} \in \mathbb{Z}_{+}$. Hence the total index sum converges.

Theorem 8.10. Every 1-efficient ideal triangulation of an anannular 3-manifold other than the solid torus or solid Klein bottle is spun 1-efficient.

Proof. This follows by a simple barrier argument. For more details see Jaco-Rubinstein [JR03] and Section 10.3. Assume that $\mathcal{T}$ is a 1-efficient triangulation of an anannular 3manifold $M$, which is not spun 1-efficient. So there is an embedded spun normal surface $S$ with $\chi(S) \geq 0$ in $M$. Clearly then $S$ is a Möbius band, annulus or disk. If $S$ is a disk, $M$ must be a solid torus or solid Klein bottle, which are excluded by assumption. So $S$ must be an annulus or Möbius band. On the other hand, if $S$ is an annulus or Möbius band, since $M$ is anannular, $S$ must be topologically boundary parallel, so is contained in a collar of a boundary component of $M$. (The Möbius band case only occurs if $M$ is nonorientable and the corresponding cusp is a Klein bottle). We can then push the appropriate boundary surface across this collar to give a torus or Klein bottle $T$ which is boundary parallel. Moreover the product region bounded by $T$ and a cusp contains $S$.

We now wish to construct a barrier using $S$. Choose a normal torus or Klein bottle $T_{0}$ which consists entirely of triangular disks and is parallel to the cusp in the previous paragraph. We can assume that $S$ and $T_{0}$ are chosen to intersect transversely. $S \cup T_{0}$ separates $M$ into various regions, with one such region $R$ homeomorphic to $M$. The boundary of $R$ contains a 'piecewise normal' torus or Klein bottle and this is a barrier for normalisation of surfaces 
in $R$ in the sense of Jaco-Rubinstein [JR03]. So we can normalise the essential torus or Klein bottle $T_{0}$ in $R$ to give an embedded normal torus or Klein bottle which is not normally peripheral, contradicting the assumption that $\mathcal{T}$ is 1-efficient.

Remark 8.11. A 1-efficient ideal triangulation of an (open) solid torus can contain an embedded spun normal disk. In fact this happens in Example 11.1 below.

A discussion of angle structures and angle structure with rotational holonomy zero on each peripheral curve follows in Appendix A.

Corollary 8.12. If $\mathcal{T}$ admits a strict angle structure with rotational holonomy zero on each peripheral curve, then it is spun 1-efficient. In particular, this applies to any geometric triangulation of a cusped hyperbolic 3-manifold.

Proof. The Euler characteristic of any embedded spun normal surface $S$ can be calculated as $\sum_{q \in \square}-\alpha(q) / \pi$, if $\alpha: \square \rightarrow(0, \pi)$ is an generalised angle structure with rotational holonomy zero on each peripheral curve. If $\alpha$ is a strict angle structure then the sum is negative, unless $S=0$.

Example 8.13. The 2-tetrahedron triangulation $\mathcal{T}$ of the trefoil knot complement in Example 11.2, is not spun 1-efficient. Here $I_{\mathcal{T}}^{0}(x \mu+y \lambda)=\delta_{0, x+6 y}$ for $x \in \mathbb{Z}, y \in \frac{1}{2} \mathbb{Z}$ so the total index $\sum_{x, y} I_{\mathcal{T}}^{0}(x \mu+y \lambda)$ diverges.

8.2. Non 1-efficient ideal triangulations. When the triangulation $\mathcal{T}$ is not 1-efficient, it turns out that the series $I_{\mathcal{T}}^{a}(b)$ may converge for some $b \neq \mathbf{0}$ even when the series $I_{\mathcal{T}}^{0}(0)$ diverges. See Example 11.4 below. The following result gives an obstruction to convergence.

Lemma 8.14. Assume that an ideal triangulation $\mathcal{T}$ contains a $Q$-normal class $S \in Q\left(\mathcal{T}_{\mathbb{Z}_{+}}\right)$ with $[S]_{2}=a$ and $\partial S$ satisfying $[\partial S]=b$, and there is a closed normal surface $0 \neq S^{\prime} \in$ $N\left(\mathcal{T} ; \mathbb{Z}_{+}\right)$such that $S^{\prime}$ is embedded and $-\chi\left(S^{\prime}\right)+2 \delta\left(S, S^{\prime}\right) \leq 0$. Then the index sum for $I_{\mathcal{T}}^{a}(b)$ diverges.

Proof. By replacing $S^{\prime}$ by $2 S^{\prime}$, if necessary, we can assume that $S^{\prime} \in Q_{0}\left(\mathcal{T}_{;} \mathbb{Z}_{+}\right)$. Then for all $k \in \mathbb{Z}_{+}$, the normal class $S_{k}=S+k S^{\prime}$ contributes a term in the index sum (30) for $I_{\mathcal{T}}^{a}(b)$ with $q^{1 / 2}$-degree

$$
\begin{aligned}
d\left(S_{k}\right) & =-\chi\left(S+k S^{\prime}\right)+\delta\left(S+k S^{\prime}\right) \\
& =-\chi(S)-k \chi\left(S^{\prime}\right)+\delta(S)+2 k \delta\left(S, S^{\prime}\right)+k^{2} \delta\left(S^{\prime}\right) \\
& =d(S)+k\left(-\chi\left(S^{\prime}\right)+2 \delta\left(S, S^{\prime}\right)\right)+k^{2} \delta\left(S^{\prime}\right) .
\end{aligned}
$$

Hence $d\left(S_{k}\right)$ remains bounded above as $k \rightarrow+\infty$ if $\delta\left(S^{\prime}\right)=0$ and $-\chi\left(S^{\prime}\right)+2 \delta\left(S, S^{\prime}\right) \leq 0$. Thus the index sum diverges.

In general, we have the following converse.

Theorem 8.15. Assume that an ideal triangulation $\mathcal{T}$ does not contain a $Q$-normal class $S \in Q\left(\mathcal{T} ; \mathbb{Z}_{+}\right)$with $\partial S=b$, and a closed normal surface $S^{\prime} \in N\left(\mathcal{T} ; \mathbb{Z}_{+}\right)$such that $S^{\prime}$ is embedded and $-\chi\left(S^{\prime}\right)+2 \delta\left(S, S^{\prime}\right) \leq 0$. Then the index sum $I_{\mathfrak{T}}^{a}(b)$ converges. 
Proof. We start by following the proof of Theorem 8.8. This shows that there exist finitely many normal classes $S_{1}, \ldots, S_{k} \in Q\left(\mathcal{T} ; \mathbb{Z}_{+}\right)$with $\partial S_{i}=b$ and finitely many normal classes $F_{1}, \ldots, F_{m} \in Q\left(\mathcal{T} ; \mathbb{Z}_{+}\right)$with $\partial F_{i}=0$ such that every $S \in Q_{b}\left(\mathcal{T} ; \mathbb{Z}_{+}\right)$with $\partial S=b$ can be written as

$$
S=S_{j}+\sum_{i=1}^{m} x_{i} F_{i}, \text { where } 1 \leq j \leq k \text { and } x_{i} \in \mathbb{Z}_{+} .
$$

Now

$$
\begin{aligned}
d(S) & =d\left(S_{j}\right)+\sum_{i}\left(-\chi\left(F_{i}\right)+2 \delta\left(F_{i}, S_{j}\right)\right) x_{i}+\sum_{i, j} \delta\left(F_{i}, F_{j}\right) x_{i} x_{j} \\
& \geq d\left(S_{j}\right)+\sum_{i}\left(-\chi\left(F_{i}\right)+2 \delta\left(F_{i}, S_{j}\right)\right) x_{i}+\delta\left(F_{i}\right) x_{i}^{2} .
\end{aligned}
$$

For each $i \in I_{0}$ we have, by assumption, either $\delta\left(F_{i}\right) \geq 1$ or $-\chi\left(F_{i}\right)+2 \delta\left(F_{i}, S_{1}\right) \geq 1$ so it follows that

$$
f_{i}(x):=\left(-\chi\left(F_{i}\right)+2 \delta\left(F_{i}, S_{1}\right)\right) x+\delta\left(F_{i}\right) x^{2}
$$

is bounded below for $x \geq 0$ and approaches $+\infty$ as $x \rightarrow+\infty$. Since there are only finitely possibilities for $S_{1}$, it follows that there are only finitely many $S \in Q_{b}(\mathcal{T} ; \mathbb{Z})$ such that $\partial S=b$ and $d(S) \leq D$. Hence the index sum for $I_{\mathcal{T}}^{a}(b)$ converges.

Remark 8.16. (1) When $b=0$ this reduces to Theorem 8.6, as we can take $S=0$ in the above theorem.

(2) If the triangulation $\mathcal{T}$ is 0 -efficient then Theorem 8.15 simplifies to the following: $I_{\mathcal{T}}^{a}(b)$ converges if and only if $\mathcal{T}$ does not contain a Q-normal class $S \in Q\left(\mathcal{T} ; \mathbb{Z}_{+}\right)$ with $\partial S=b$, and an embedded closed normal surface $S^{\prime}$ disjoint from $S$ such that $\chi\left(S^{\prime}\right)=0$.

\section{INVARIANCE UNDER 2-3 AND 0-2 MOVES}

The arguments of [GHRS15] extend easily to prove the following.

Theorem 9.1. Let $\mathcal{T}$ be an ideal triangulation of $M$ and let $\tilde{\mathcal{T}}$ be obtained from $\mathcal{T}$ by a $2-3$ move. Then for all $a, b$ as above, $I_{\mathcal{T}}^{a}(b)=I_{\tilde{\mathfrak{T}}}^{a}(b)$ provided both sides are defined.

Proof. We follow the argument from [GHRS15, Theorem A.1], indicating the main changes needed in the current setting. Consider a $2-3$ move from $\mathcal{T}$ to $\tilde{\mathcal{T}}$; this occurs in a bipyramid consisting of 2 tetrahedra in $\mathcal{T}$ which are replaced by 3 tetrahedra sharing an edge of order 3 in $\tilde{\mathcal{T}}$.

First note that each $Q$-normal class $S \in Q(\mathcal{T} ; \Sigma)$ can be written as a linear combination of edge solutions, tetrahedral solutions and peripheral curve solutions with coefficients in $\frac{1}{2} \mathbb{Z}$, by the results of Section 6 (see Remark 7.6). As in section 4.4 of [GHRS15], we can represent $S$ by a linear combination $\omega$ of oriented normal arcs in the induced triangulation $\mathcal{T}_{\partial}$ of $\partial M$, chosen so that each quad coordinate for $S$ is a sum of 4 "turning numbers" of normal arcs, coming from the 4 corners of the tetrahedron containing the quad (see figure 7 of [GHRS15]). 
Explicitly, for each edge solution we choose a small normal linking circle in $\partial M$ around one end of the edge in $\mathcal{T}$, we choose oriented normal curves representing each peripheral class, and for each tetrahedral solution we choose three normal arcs oriented anticlockwise in a triangle coming from one corner of the tetrahedron. (If tetrahedral solutions are needed in the bipyramid, we choose the two triangles at the degree 3 vertices at the top and bottom.) One difference from [GHRS15] is that the coefficients of the normal arcs here can be halfintegers; but each sum of turning numbers giving a quad coefficient is still an integer.

To compute the index $I_{\mathcal{T}}^{a}(b)$, we choose a normal class $S_{0}$ with $\left[S_{0}\right]_{2}=a$ and $\left[\partial S_{0}\right]=b$, and have a contribution from each normal class

$$
S_{\boldsymbol{k}}=S_{0}+\sum k_{i} E_{i}
$$

where $\boldsymbol{k}=\left(k_{i}\right) \in \mathbb{Z}^{n-r}$ is a set of integer weights on a set of $n-r$ "basic edges". (The excluded edges are taken from a "maximal tree with 1 - or 3 - cycle for $\mathcal{T}$ " as in Theorem 4.3 of [GHRS15]; this ensures that the map $\boldsymbol{k}=\left(k_{i}\right) \mapsto \sum k_{i} E_{i}+\mathbb{T}$ is an isomorphism from $\mathbb{Z}^{n-r}$ onto $(\mathbb{E}+\mathbb{T}) / \mathbb{T}$.) We can represent $S_{0}$ by a linear combination $\omega$ of oriented normal arcs as described above. By adding $k_{i}$ small linking circles around one end of the $i$ th edge in $\mathcal{T}$ we obtain a linear combination $\omega_{\boldsymbol{k}}$ of oriented normal arcs which gives the quad coordinates of $S_{\boldsymbol{k}}=S_{0}+\sum k_{i} E_{i}$

The construction of [GHRS15] shows how to replace $\omega$ by a linear combination $\tilde{\omega}$ of oriented normal arcs in $\tilde{\mathcal{T}}_{\partial}$ representing a normal class $\tilde{S}_{0}$ in $\tilde{\mathcal{T}}$ and this satisfies $\left[\tilde{S}_{0}\right]_{2}=\left[S_{0}\right]_{2}$ and $\left[\partial S_{0}\right]=\left[\partial \tilde{S}_{0}\right]$. (Essentially, $\tilde{S}_{0}$ is obtained by subdividing the normal disks of $S_{0}$, so its homology classes do not change.) Then the index sum for $I_{\tilde{\mathfrak{T}}}^{a}(b)$ has a contribution for each normal class

$$
\tilde{S}_{\tilde{\boldsymbol{k}}}=\tilde{S}_{0}+k_{0} \tilde{E}_{0}+\sum k_{i} \tilde{E}_{i}
$$

where $k_{0}$ is the integer weight on the new edge class $\tilde{E}_{0}$ in $\tilde{\mathcal{T}}$, and $\boldsymbol{k}=\left(k_{i}\right) \in \mathbb{Z}^{n-r}$ as above give the weights on the edge classes $\tilde{E}_{i}$ coming from $\mathcal{T}$. Adding small linking circles around one end of each edge in $\mathcal{T}$ gives a linear combination of normal $\operatorname{arcs} \tilde{\omega}_{\tilde{\boldsymbol{k}}}$ which gives the quad coordinates of $\tilde{S}_{\tilde{\boldsymbol{k}}}$.

The proof of the Pentagon Equality (Lemma A.3 in [GHRS15]) now goes through verbatim; this gives the result. The only difference in the current setting is that coefficients of normal arcs and turning numbers can now be half-integers. However since the sums giving quad coefficients used in the tetrahedral index functions are integers, the arguments of [GHRS15] go through without change.

The arguments from [GHRS15] also extend to give the following.

Theorem 9.2. Let $\mathcal{T}$ be an ideal triangulation of $M$ and let $\tilde{\mathcal{T}}$ be obtained from $\mathcal{T}$ by a 0-2 move. Then for all $a, b$ as above, $I_{\mathcal{T}}^{a}(b)=I_{\tilde{\mathfrak{T}}}^{a}(b)$ provided both sides are defined.

Proof. Here the proof of Theorem 5.1 in [GHRS15] applies, if we start with a linear combination $\omega$ of oriented normal arcs in $\mathcal{T}_{\partial}$ with $\frac{1}{2} \mathbb{Z}$ coefficients giving the quad coordinates of $S_{0}$ with homology classes $\left[S_{0}\right]_{2}=a$ and $\left[\partial S_{0}\right]=b$. 


\section{Generalised nORMal SURFACES}

Embedded normal surfaces were introduced by Kneser [Kne29] in 1929. In the 1950s and 1960s, Haken [Hak61b, Hak61a, Hak68] extensively developed the theory of normal surfaces (via handle decompositions and also via triangulations) and applied this to basic algorithmic questions in topology. The solutions to Haken's matching equations include branched and immersed normal surfaces, which are the result of gluing together finitely many normal disks in tetrahedra of a triangulation of a closed manifold or an ideal triangulation of a compact manifold with boundary. In the 1980s Thurston suggested the theory of spun normal surfaces (see [Til08, Wal11]). These are obtained by gluing finitely many quadrilaterals but possibly infinitely many triangular disks, as long as there are at most a finite number of such disks which are not contained in 'tails' which are infinite annuli spiralling around a cusp. We will again specify if a spun normal surface is embedded - in general it can be branched or immersed. Moreover spun normal surfaces may contain no tails, in which case they are (closed) normal surfaces.

In this section we discuss generalised normal surfaces and normal classes. The former have been called $k$-normal surfaces, spinal surfaces and helicoidal surfaces in the literature (see [Mat03, FM09, FKB08, Bac]). Our aim is to show the interesting connections both with normal surface theory and with the duality between homology and cohomology. Moreover the lattices over which the 3D-index is evaluated can be represented as collections of embedded generalised normal surfaces, lying in a particular $\mathbb{Z} / 2 \mathbb{Z}$-homology class. Finally generalised normal surface theory can be viewed as a projection of normal surface theory, with the subspace of solutions spanned by the tetrahedral solutions quotiented out.

10.1. Generalised normal and spun normal surfaces. Firstly, we discuss normal curve theory (with real coefficients) on the 2-sphere equipped with its four simplex triangulation as the boundary of a tetrahedron $\Delta^{3}$. We just recall the key points (see [Mat03, Section 3.2] for a detailed exposition).

- There are 12 normal arc types in $\partial \Delta^{3}$ and so normal curves are defined by nonnegative integer vectors in $\mathbb{R}^{12}$ satisfying 6 matching equations. It is easy to see that edge weights of normal curves determine the numbers of normal arcs and there are six independent edge weights. Hence the solution space $W(\mathbb{R})$ to the matching equations is 6-dimensional.

- For the integer solution space $W(\mathbb{Z})$ to the matching equations, the only additional constraints on the edge weights are mod 2 conditions. Namely, the three edges weights at a face are either all even or two are odd and one even.

- There are precisely 7 vertex classes of the projective solution space $\mathcal{P}$, which is a 5 -dimensional polytope. These vertices are the classes of the 4 boundaries of triangular normal disks and the 3 boundaries of quadrilateral normal disks. They satisfy precisely one relation: that the sum of the triangular curves is the sum of the quadrilateral curves.

- There are 21 facets of $\mathcal{P}$ which are all 4-simplices. Namely, there are 12 facets containing 3 quadrilateral curves and 2 triangular curves as vertices, 3 facets containing 4 quadrilateral curves and 1 triangular curve, and 6 facets containing 2 quadrilateral curves and 3 triangular curves. 
Next, we want to analyze the quotient space $N(\mathcal{T} ; \mathbb{Z}) / \mathbb{T}$ of integer normal classes modulo tetrahedral solutions, working in quadrilateral space.

For each tetrahedron, this is equivalent to studying the normal arc solution space $W(\mathbb{Z})$ modulo the $\mathbb{Z}$-submodule spanned by the 4 triangular curves in the boundary of the tetrahedron. This is a 2-dimensional space generated by the coset representatives of any 2 of the 3 quadrilateral curves. So elements can be viewed as integer linear combinations of any 2 quadrilateral curves.

We can represent the tetrahedron as a square pillowcase, triangulated with 2 triangles on the top and 2 triangles on the bottom, and choose normal coordinates so that the 3 quadrilateral curves correspond to $(1,0),(0,1),(1,1) \in \mathbb{Z}^{2}$. Now it is easy to see what happens when we take a linear combination $p(1,0)+q(0,1)=(p, q)$ where $p, q \in \mathbb{Z}_{+}$. If $p$ and $q$ are relatively prime, then the $(p, q)$ normal class can be represented by a simple closed geodesic of slope $q / p$ on a square pillowcase with edge weights $p, q, p+q$ as shown in Figure 9 below. In general, if $p, q$ are not relatively prime, we get $d$ parallel copies of a simple closed normal curve where $d=\operatorname{gcd}(p, q)$.

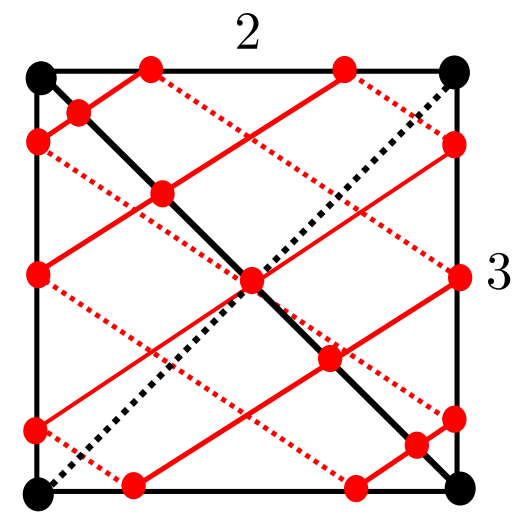

Figure 9. A $(3,2)$ normal curve on the square pillowcase, with edge weights 3,2 and 5 along the tetrahedron edges.

Alternatively, if we lift to the 2-fold branched covering of the 2-sphere over the 4 vertices, the result is a 2 -torus. Then the $(p, q)$ normal curve lifts to simple closed $(p, 2 q)$ curves on the torus.

Definition 10.1. A generalised normal disk in a tetrahedron is a properly embedded disk whose boundary is an embedded simple closed normal curve.

Remark 10.2. These generalised normal disks arise naturally in the theory of Dehn filling of tangles, introduced by Montesinos in [Mon73]. Namely, the generalised normal disks are the disks that separate the two strands of a rational tangle.

Definition 10.3. A generalised normal surface in an ideal triangulation $\mathcal{T}$ of a compact 3 -manifold is an embedded surface which meets each tetrahedron of $\mathcal{T}$ in a finite collection of disjoint generalised normal disks.

Remark 10.4. Special cases of this notion have been considered previously, for example in [Mat03, FM09, FKB08, Bac]. 
Lemma 10.5. Given a closed normal class $S$ in $\tilde{N}\left(\mathcal{T} ; \mathbb{Z}_{+}\right)$with at most two non-zero quadrilateral coordinates in each tetrahedron, there is an embedded generalised normal surface $\tilde{S}$ with the same edge weights, which is unique up to normal isotopy.

Proof. This is essentially the same argument as the construction of a closed normal surface from its triangle and quad coordinates (see for example [Mat03]). Any solution $S \in \tilde{N}\left(\mathcal{T} ; \mathbb{Z}_{+}\right.$) to Haken's matching equations gives an embedded collection of normal arcs in the 2-skeleton of the triangulation which is unique up to normal isotopy: first choose $w(e)$ disjoint points in the each edge $e$ where the "weight" $w(e)$ is the number of normal disks meeting edge $e$, and extend this to an embedded collection of normal arcs in each triangle. The arc pattern on the boundary of each tetrahedron can then can be filled in uniquely (up to normal isotopy) by adding generalised normal disks and normal triangles. The matching equations imply that these fit together to give a generalised normal embedded surface.

We can also consider generalised spun normal surfaces in an ideal triangulation $\mathcal{T}$, which meet each ideal tetrahedron in a finite collection of disjoint generalised normal disks and possibly infinitely many triangular normal disks.

Lemma 10.6. Given a $Q$-normal class $S$ in $Q\left(\mathcal{T} ; \mathbb{Z}_{+}\right)$with at most two non-zero quadrilateral coordinates in each tetrahedron, there is a unique embedded generalised spun normal surface $\tilde{S}$ such that the edge weights of the non-triangular disks in $S$ and $\tilde{S}$ agree.

Proof. This is analogous to the construction of a spun normal surface from a solution $S$ to the Q-matching equations, as described in [Til08] (see also [DG12]). We first choose $w(e)$ disjoint points in the each edge $e$ where $w(e)$ is the weight of quadrilateral normal disks of $S$ meeting edge $e$, and extend this to an embedded collection of normal arcs in each triangle. Next add infinitely many parallel arcs at each corner of each triangle. The arc pattern on the boundary of each tetrahedron can then can be filled in uniquely (up to normal isotopy) by adding generalised normal disks and infinitely many normal triangles. There is a unique way to glue together the faces of tetrahedra in pairs in the given combinatorial pattern so the normal arcs match, and the $Q$-matching equations show that the generalised normal disks fit together consistently (without any "shearing") around each edge class. Removing any boundary parallel normal surface components gives the desired embedded generalised spun normal surface $\tilde{S}$. (If $\partial S=0$, the result is an embedded closed normal surface.)

Remark 10.7. In the above two lemmas, $\tilde{S}$ is connected if the class $S$ is irreducible.

The normal classes needed for the summation to get the 3D-index correspond to embedded generalised normal surfaces. The correspondence is unique in the sense that the process produces a unique embedded generalised normal or spun normal surface with at most two non-zero quadrilateral coordinates in each tetrahedron. This gives bijections

$[S]=S+\mathbb{T} \in Q(\mathcal{T} ; \mathbb{Z}) / \mathbb{T} \leftrightarrow S^{*} \in Q\left(\mathcal{T} ; \mathbb{Z}_{+}\right) \leftrightarrow \tilde{S} \in\{$ embedded generalised spun normal surfaces $\}$

10.2. Degrees of index terms. Recall, from equation (32), that the $q^{1 / 2}$-degree of a term $I([S])$ in the $q$-series for the $3 \mathrm{D}$-index is given by the expression

$$
d(S)=d\left(S^{*}\right)=-\chi\left(S^{*}\right)+\delta\left(S^{*}\right),
$$


where $S^{*}$ is the minimal positive coset representative for $[S]=S+\mathbb{T}$. Then $S^{*}$ is a normal class with at most two quadrilateral types in each tetrahedron, and

$$
d\left(S^{*}\right)=-\chi\left(S^{*}\right)+\sum_{i} p_{i} q_{i}
$$

where $p_{i}, q_{i} \geq 0$ are the numbers of quadrilaterals for $S^{*}$ in the $i t h$ tetrahedron, and $\chi\left(S^{*}\right)$ denotes the formal Euler characteristic of $S^{*}$.

Lemma 10.8. Let $S \in Q(\mathcal{T} ; \mathbb{Z})$ be a Q-normal class and let $\tilde{S}$ be the embedded generalised normal surface $\tilde{S}$ corresponding to $S^{*}$. Then

$$
d(S)=-\chi(\tilde{S})+\sum_{i}\left(p_{i} q_{i}-p_{i}-q_{i}+\operatorname{gcd}\left(p_{i}, q_{i}\right)\right)
$$

where $p_{i}, q_{i} \geq 0$ are the numbers of quadrilaterals for $S^{*}$ in the ith tetrahedron.

Hence $d(S) \geq-\chi(\tilde{S})$ with equality if and only if $\tilde{S}$ is a "special" embedded generalised normal surface, i.e. in each tetrahedron one of the coefficients $p_{i}$ or $q_{i}$ is either 0 or 1.

Proof. We can choose a generalised angle structure on $\mathcal{T}$ with vanishing rotational peripheral holonomy by Proposition A.2, and use this to compare the Euler characteristic of $\tilde{S}$ with the formal Euler characteristic of $S^{*}$, using Proposition A.4 and Definition A.6 from Appendix A.

In both cases we get corner terms of (formal angles) $/ 2 \pi$ which add to 1 for each vertex. Moreover the number of edges in $\tilde{S}$ is the same as for $S^{*}$, but the number of disks is reduced from $\sum_{i}\left(p_{i}+q_{i}\right)$ to $\sum_{i} \operatorname{gcd}\left(p_{i}, p_{i}\right)$. So $\chi(\tilde{S})=\chi\left(S^{*}\right)+\sum\left(\operatorname{gcd}\left(p_{i}, q_{i}\right)-p_{i}-q_{i}\right)$. Hence $d\left(S^{*}\right)=-\chi\left(S^{*}\right)+\sum_{i} p_{i} q_{i}=-\chi(\tilde{S})+\sum_{i}\left(p_{i} q_{i}-p_{i}-q_{i}+\operatorname{gcd}\left(p_{i}, q_{i}\right)\right)$.

The final result now follows from the observation that for any integers $p, q \geq 0$ we have

- $p q-p-q+\operatorname{gcd}(p, q)=0$ if $p$ or $q$ is 0 , and otherwise

- $p q-p-q+\operatorname{gcd}(p, q) \geq p q-p-q+1=(p-1)(q-1) \geq 0$ with equality if and only if $p$ or $q$ is 1 .

Remark 10.9. A very similar function is studied in [FKB08, Section 4.1].

Remark 10.10. We will be particularly interested in terms in the 3D-index sum which have non-positive degrees.

10.3. Normalisation and barriers. There is a well-known procedure going back to Kneser [Kne29] for 'normalisation' of an embedded closed surface $S$ in a triangulation $\mathcal{T}$ of a manifold $M$. We can assume initially that the surface $S$ is transverse to the triangulation. There are five basic moves:

(1) Compress $S$ along an embedded disk $D$ in the interior of a tetrahedron $\Delta$, which meets $S$ in $C=\partial D$, where either $C$ is essential in $S$ or a disk bounded by $C$ in $S$ meets $\partial \Delta$.

(2) Isotope a disk $D^{\prime}$ of intersection of $S$ with a tetrahedron across a face of the tetrahedron, where $\partial D^{\prime}$ lies in a face and is an innermost curve in that face. 
(3) Isotope an innermost arc of intersection of $S$ with a face of the triangulation across an edge. Here the arc has both ends on the edge and there are no intersections of $S$ with the bigon cut off by the arc in the face.

(4) Boundary compress the intersection of $S$ with a tetrahedron. This occurs along a bigon which intersects $S$ in one arc of its boundary with the other arc contained in an edge of the tetrahedron.

(5) Finally, any component which is a sphere contained in the interior of a tetrahedron is removed.

Kneser [Kne29] and Haken [Hak61b] show that any surface can either be normalised, i.e. either converted to a non-empty normal surface by a sequence of such moves, or else converted to the empty surface.

Remark 10.11. If an embedded sphere $S$ or an embedded torus $T$ is converted to the empty surface when normalised in one of its complementary regions, then that region is a 3 -ball or solid torus in $M$ bounded by $S$ or $T$ respectively.

In fact, if $S$ or $T$ did not bound a 3-ball or solid torus respectively then there is no isotopy or a disk compression followed by an isotopy, shrinking $S$ or $T$ respectively to a point.

Definition 10.12. An embedded surface $S^{\prime}$ is a barrier for the normalisation of $S$ if it satisfies the following conditions. Firstly, $S^{\prime} \cap S=\emptyset$. Secondly, $S$ can be normalised by moves in the complement of $S^{\prime}$.

Theorem 10.13. Let $\mathcal{T}$ be an ideal triangulation of compact manifold with boundary consisting of tori. Suppose that $S^{\prime}$ is a "simple" embedded generalised normal surface in $\mathcal{T}$, i.e. has at most one generalised normal disk in each tetrahedron. Then $S^{\prime}$ is a barrier for normalisation of any surface in its complement.

Proof. Consider the two regions $R, R^{\prime}$ obtained by splitting a tetrahedron $\Delta$ open along a generalised normal disk $D$ in $S^{\prime}$. The faces of these regions consist of a copy of $D$ and the result of splitting the faces of $\Delta$ open along $\partial D$. The latter produce faces which are either triangles, quadrilaterals or pentagons. (Hexagons cannot arise since a generalised normal disk has at most two different arc types in any triangular face of $\Delta$.)

Choose a maximal family of disjoint non parallel properly embedded bigon compressing disks for each of the regions $R, R^{\prime}$, as in the fourth normalisation move. Thus, a quadrilateral face of $R$ or $R^{\prime}$ has two associated bigon disks, one along each edge of $\Delta$ in its boundary, whereas a pentagon has one such bigon. (Figure 10 exhibits the case where $D$ is a 12 -gon, with $\partial \Delta$ stereographically projected to the plane.)

If either region is compressed along all these disks, each of the resulting components is either a tetrahedron, where the compression of $D$ gives a face of the tetrahedron, or a 3-ball with boundary a pair of disks, where one disk is the result of compressing $D$ and the other disk is in a face of $\Delta$.

Suppose that $S$ is a surface disjoint from $S^{\prime}$. If $S$ meets either region $R, R^{\prime}$, then notice that the intersection of $S$ with the above families of bigon disks can be used to perform normalisation moves on $S$ in the complement of $S^{\prime}$. For if there is a loop $C$ in $S \cap D_{i}$ where 

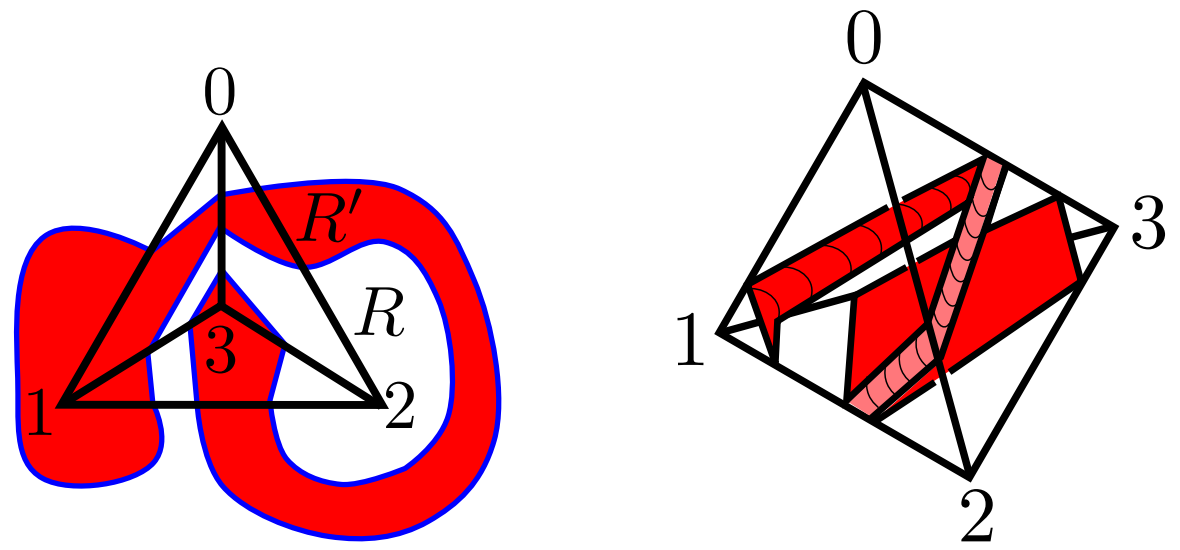

Figure 10. (left) A schematic diagram of the 12-gon determined by coordinates $(2,1,0)$. (right) An embedding of this 12 -gon. The region $R$ faces the dark (red) shaded side, while $R^{\prime}$ faces the lighter side.

$D_{i}$ is one of the bigon disks and either $C$ is essential on $S$ or a disk bounded by $C$ in $S$ meets $\partial \Delta$, then this gives a first normalisation move. On the other hand, if there are arcs in $S \cap D_{i}$, these give third or fourth normalisation moves.

After performing all such normalisation moves, the resulting surface again denoted by $S$ is still disjoint from $S^{\prime}$ but also from all the bigon disks in $R, R^{\prime}$. It is easy to see that we can perform additional first or second normalisation moves so that $S \cap \Delta$ consists entirely of triangular normal disks and spheres in interior $\Delta$ and after discarding any sphere components we have normalised $S$ in $\Delta$.

This argument can clearly be performed in all tetrahedra containing a generalised normal disk, and in tetrahedra containing only normal disks, the argument in [JR03] applies. This completes the proof.

Corollary 10.14. If $S^{*}$ is a two-sided embedded "simple" generalised normal surface (i.e. has at most one generalised normal disk in each tetrahedron), then it can be pushed off itself to either side and normalised in its complement.

Remark 10.15. If we consider an arbitrary generalised normal surface $S^{*}$ which is not necessarily simple, the same idea shows that any surface $S$ in the complement of $S^{*}$ can be transformed into a generalised normal surface, by moves similar to the ones above for normalisation. The difference in this case is that if $S^{*}$ has parallel generalised normal disks bounding a product region $R$ in a tetrahedron and $S$ meets $R$, then $S$ might be transformed to intersect $R$ in parallel copies of these generalised normal disks.

Theorem 10.16. Let $\mathcal{T}$ be a 1-efficient ideal triangulation of a cusped, orientable 3-manifold $M$, and assume that $M$ is not a solid torus or $T^{2} \times I$. Then $\mathcal{T}$ contains no closed embedded simple generalised normal surface $\tilde{S}$ with $\chi(\tilde{S}) \geq 0$.

Proof. Recall that $\mathcal{T}$ 1-efficient implies that $M$ is irreducible and atoroidal. Suppose first that $\tilde{S}$ is two-sided in $M$. Now by the barrier argument in Corollary 10.14, we can push the simple generalised surface $\tilde{S}$ off itself on either side, and normalise it. The result is either (i) a normal sphere, or (ii) a normal torus, or else (iii) the surface becomes empty. 
The first case clearly contradicts the assumption of 1-efficiency.

In the second case, note that the surface may normalise to a peripheral torus at a cusp when it is pushed off itself to a side containing a cusp. Since we are assuming that $M$ is not $T^{2} \times I$, it follows that normalisation to a peripheral torus cannot occur on both sides of a generalised normal torus. But then, pushing off to at least one side, we would get a normal torus which is topologically but not normally parallel into a cusp, again contradicting 1-efficiency.

So we are left with the case that the surface becomes empty. If $\tilde{S}$ is a sphere, it cannot bound a ball on both sides and hence yields a normal sphere on one side or the other by Remark 10.15, contrary to assumption.

If $\tilde{S}$ is a torus, it could be compressible. In this case, the torus bounds a solid torus or cube-with-knotted-hole. In the latter case, the sphere resulting from compressing $\tilde{S}$ does not bound a ball in the complement of $\tilde{S}$ so can be normalised giving a normal sphere, contrary to assumption. So we are left with the final case, where $\tilde{S}$ is a torus bounding a solid torus. The other side of $\tilde{S}$ must contain a cusp of $M$. But then if $\tilde{S}$ is compressible on this side, the result is a sphere which can be normalised, contradicting the assumption of 1-efficiency. On the other hand, if $\tilde{S}$ is incompressible on the side containing a cusp, either $\tilde{S}$ normalises to give a non-peripheral normal torus, contrary to 1-efficiency, or $M$ is a solid torus, which is excluded by our hypothesis. So the case where $\tilde{S}$ is two-sided is complete.

If the generalised normal surface $\tilde{S}$ is one-sided it is a projective plane or Klein bottle. The first case contradicts irreducibility. If $\tilde{S}$ is a Klein bottle, the boundary of a small regular neighbourhood of $\tilde{S}$ is either an incompressible torus, contradicting the atoroidal assumption, or compressible and $M$ is a closed prism manifold, contradicting the hypothesis that $M$ has a cusp. This completes the proof.

Lemma 10.17. Let $\mathcal{T}$ be a 1-efficient ideal triangulation of $M$, which is either a solid torus or $T^{2} \times I$. Then $\mathcal{T}$ contains no closed embedded simple generalised normal surface $\tilde{S}$ with $\chi(\tilde{S})>0$, and no closed embedded normal surface $\tilde{S}$ with $\chi(\tilde{S})=0$ which is non-peripheral. However, $\mathcal{T}$ always contains a closed embedded generalised normal $\tilde{S}$ with $\chi(\tilde{S})=0$. In fact, there is an almost normal torus $\tilde{S}$ containing one octagonal disk.

Proof. If there was a simple generalised normal surface $\tilde{S}$ with $\chi(\tilde{S})>0$, then $\tilde{S}$ would be a sphere or projective plane. A projective plane can be normalised and in the case of a sphere, $\tilde{S}$ could be normalised to the side which was not a ball. Both cases contradict 1-efficiency. Similarly there cannot be a non-peripheral normal surface $\tilde{S}$ with $\chi(\tilde{S})=0$ for the same reason.

By a standard sweepout argument, starting with a peripheral normal torus at a cusp and sweeping to a core circle or the other cusp, there must be an intermediate almost normal torus $\tilde{S}$ - see Rubinstein [Rub97] or Stocking [Sto00]. If $\tilde{S}$ was obtained by attaching a tube to a normal sphere, this would contradict 1-efficiency. Hence $\tilde{S}$ must have one octagonal disk as claimed.

Remark 10.18. The proof of Theorem 10.16 shows that a 1-efficient triangulation $\mathcal{T}$ of a closed orientable 3-manifold $M$ other than $S^{3}$ or a lens space contains no closed embedded simple generalised normal surface $\tilde{S}$ with $\chi(\tilde{S}) \geq 0$. (Here 1-efficient means there are no 
embedded normal spheres, projective planes, Klein bottles or tori, except for vertex linking spheres and edge linking tori. The former are boundaries of a small regular neighbourhood of a vertex, and the latter are obtained similarly from an edge which is a loop.)

Corollary 10.19. Let $\mathcal{T}$ be a 1-efficient ideal triangulation of a manifold $M$ other than the solid torus or $T^{2} \times I$, and let $0 \neq S \in N(\mathcal{T} ; \mathbb{Z})$ be an irreducible closed normal class. Then the contribution of $S$ to the $3 D$-index sum has $q^{1 / 2}$-degree $d(S) \geq 1$.

Proof. Since $S$ is irreducible, it contains no tetrahedral solution summand, so $S=S^{*}$ is the minimal non-negative coset representative of $[S]=S+\mathbb{T}$. Then $S^{*}$ contains at most two quad types in each tetrahedron, so can be replaced by an embedded generalised normal surface $\tilde{S}$ by Lemma 10.5. Now $\tilde{S}$ is connected since $S$ is irreducible, and $d(S) \geq-\chi(\tilde{S})$ by Lemma 10.8. Assume first that $\tilde{S}$ is not simple, so there are at least two of two different quadrilateral types in some tetrahedron for $S^{*}$. But then Lemma 10.8 implies that $d(S) \geq 2$. On the other hand, if $\tilde{S}$ is simple and $M$ is not a solid torus, then $\chi(\tilde{S})<0$ by Theorem 10.16 , hence $d(S) \geq 1$. This completes the proof.

Remark 10.20. This result fails for 1-efficient triangulations of the solid torus and $T^{2} \times I$. Here every normal class $S$ contributing to the index sum has degree $d(S) \geq 0$, but an almost normal torus $S$ gives a contribution $d(S)=0$. (Compare Example 11.1 below.)

One consequence of Corollary 10.19 and Remark 10.20 is the following basic fact about the 3D-index.

Corollary 10.21. If $\mathcal{T}$ is a 1-efficient ideal triangulation then the $3 D$-index $I_{\mathcal{T}}^{0}(0)$ is a formal power series in $q^{1 / 2}$, not just a formal Laurent series in $q^{1 / 2}$.

In general, we expect that the minimum $q^{1 / 2}$-degree $d_{\text {min }}$ of $I_{\mathcal{T}}^{0}(k b)$ grows quadratically as $k \rightarrow+\infty$. However this is no longer true if $b$ is the boundary of an embedded spun normal surface.

Lemma 10.22. Assume $\mathcal{T}$ is a 1-efficient triangulation of a 3-manifold other than the solid torus or $T^{2} \times I$. Assume that $\mathcal{T}$ contains an embedded spun normal surface $S$ with $\partial S=b \neq 0$, and that $S$ is the only irreducible spun normal class in $Q\left(\mathcal{T} ; \mathbb{Z}_{+}\right)$whose boundary is a positive integer multiple of $b$. Then the minimum $q^{1 / 2}$-degree $d_{\min }$ of $I_{\mathcal{T}}^{0}(k b)$ grows at mostly linearly as $k \rightarrow+\infty$. In fact, $d_{\text {min }} I_{\mathcal{T}}^{0}(k b) \leq-\chi(S) k$ for all even $k \geq 0$.

Proof. For each even $k \geq 0, k S$ has $\partial(k S)=k b$ and $[k S]=0$ so contributes a term to $I_{\mathcal{T}}^{0}(k b)$ with $\left.d(k S)^{*}\right)=d(k S)=-\chi(S) k+\delta(S) k^{2}=-\chi(S) k$. Now, by our assumption, any $S^{\prime}$ with $\partial S^{\prime}=b$ can be written as $S^{\prime}=k S+S_{0}$ where $\partial S_{0}=0$. Hence $d\left(S^{\prime}\right) \geq d(k S)+d\left(S_{0}\right)>d(k S)$ unless $S_{0}=0$ by Corollary 10.19 .

Example 10.23. For the figure eight knot complement, with its canonical triangulation $\mathcal{T}$ given in Example 4.1, there are embedded, essential, spun normal once-punctured Klein bottles with boundary $b= \pm 4 \mu \pm \lambda$ and Euler characteristic $\chi=-1$ giving a term in $I_{\mathcal{T}}^{0}(k b)$ of degree $-\chi k=k$ for each $k \geq 0$. Further this is the only irreducible spun normal class with boundary a positive multiple of $b$, so by Lemma 10.22 this is the minimum $q^{1 / 2}$-degree of $I_{\mathcal{T}}^{0}(k b)$. (Compare the calculations for $I_{\mathcal{T}}^{0}(8 \mu+2 \lambda)=I_{\mathcal{T}}(4 \mu+\lambda)$ and $I_{\mathcal{T}}^{0}(4 \mu+\lambda)=I_{\mathcal{T}}\left(2 \mu+\frac{1}{2} \lambda\right)$ given in Example 4.1.) 
For the trefoil knot complement, with its 2 tetrahedra triangulation $\mathcal{T}$ given in Example 11.2, we have $I_{\mathcal{T}}^{0}((x \mu+y \lambda))=\delta_{0, x+6 y}$. Here there is an embedded, essential, spun normal Möbius strip $S$ with boundary $b= \pm(6 \mu-\lambda)$ giving a contribution to $I_{\mathcal{T}}^{0}(k b)$ of degree $0=-\chi(S) k$ for all $k \in \mathbb{Z}_{+}$. Further, this is the only irreducible spun normal class with boundary a positive multiple of $b$, so Lemma 10.22 again applies.

\section{Some EXAmples}

In this section we give some examples of index computations for ideal triangulations with 2 tetrahedra, taken from the census of triangulations described in Section 12 below.

11.1. Solid torus. The (open) solid torus has a 1-efficient triangulation $\mathcal{T}$ by 2 tetrahedra with isomorphism signature 'cMcabbgds', and gluing equation/holonomy coefficient matrix (from SnapPy) given by:

$$
\left[\begin{array}{cccccc}
2 & 1 & 2 & 2 & 2 & 2 \\
0 & 1 & 0 & 0 & 0 & 0 \\
0 & 1 & 0 & 0 & -1 & 0 \\
0 & 1 & 0 & 0 & 0 & 2
\end{array}\right]
$$

(Here the peripheral curves $\mu, \lambda$ correspond to the meridian and longitude for the unknot in $S^{3}$.) Summing over integer weights $k$ on edge 2 gives the 3D-index

$$
\begin{aligned}
I_{\mathcal{T}}(x \mu+y \lambda) & =\sum_{k \in \mathbb{Z}} q^{k} J_{\Delta}(0, k+x+y, 0) J_{\Delta}(0,-x, 2 y) \\
& =J(0,-x, 2 y) q^{-x-y} \sum_{k \in \mathbb{Z}} q^{k} J(0, k, 0)=0
\end{aligned}
$$

since

$$
\sum_{k \in \mathbb{Z}} q^{k} J_{\Delta}(0, k, 0)=\sum_{k \in \mathbb{Z}} I_{\Delta}(0, k) q^{k}=I(0, q, q)=\frac{\left(q q^{-1} ; q\right)_{\infty}}{(q)_{\infty} ; q}=0
$$

using equation (2).

It follows that the 3D-index vanishes identically for all 118753 1-efficient ideal triangulations of the solid torus with at most 6 tetrahedra, since we have checked that these are all connected to $\mathcal{T}$ by 2-3, 3-2, 0-2 and 2-0 moves preserving 1-efficiency.

11.2. Trefoil complement. The right-handed trefoil complement ('L103001' in SnapPy) has a 2-tetrahedron triangulation $\mathcal{T}$ with gluing equation/holonomy coefficients given by:

$$
\left[\begin{array}{cccccc}
1 & 2 & 2 & 2 & 1 & 2 \\
1 & 0 & 0 & 0 & 1 & 0 \\
0 & 0 & -1 & 1 & 0 & 0 \\
1 & 0 & -4 & 4 & -1 & 0
\end{array}\right]
$$


36 STAVROS GAROUFALIDIS, CRAIG D. HODGSON, NEIL R. HOFFMAN, AND J. HYAM RUBINSTEIN

Hence, summing over edge 2 , the $3 \mathrm{D}$-index is

$$
\begin{aligned}
I_{\mathcal{T}}(x \mu+y \lambda) & =\sum_{k \in \mathbb{Z}} J_{\Delta}(k+y, 0,-x-4 y) J_{\Delta}(x+4 y, k-y, 0) \\
& =\sum_{k} q^{k} I_{\Delta}(x+4 y, k+y)\left(-q^{1 / 2}\right)^{-x-4 y} I_{\Delta}(x+4 y, k-x-5 y) \\
& =\sum_{k^{\prime}} q^{k^{\prime}}\left(-q^{1 / 2}\right)^{-x-6 y} I_{\Delta}\left(m, k^{\prime}\right) I_{\Delta}\left(m, k^{\prime}-x-6 y\right) \\
& =\sum\left(-q^{1 / 2}\right)^{-x-6 y} \delta_{0,-x-6 y}=\delta_{0, x+6 y} .
\end{aligned}
$$

where we put $k^{\prime}=k+y$ and $m=x+4 y$ and used the quadratic identity.

Note that $I_{\mathcal{T}}(\gamma)$ is non-trivial exactly when $\gamma$ is a multiple of the boundary curve $-6 \mu+\lambda$ of the essential annulus (and Möbius strip) in the trefoil knot exterior.

11.3. $\mathbf{T}^{\mathbf{2}} \times \mathbf{I}$. There is a unique triangulation of $T^{2} \times I$ with 3 tetrahedra. Regina's isomorphism signature of the triangulation is 'dLQacccbjkg'. This triangulation turns out to be 1-efficient and has the following gluing equation/holonomy coefficients (from SnapPy):

$$
\left[\begin{array}{ccccccccc}
1 & 2 & 2 & 1 & 1 & 1 & 1 & 1 & 1 \\
1 & 0 & 0 & 0 & 0 & 0 & 0 & 0 & 0 \\
0 & 0 & 0 & 1 & 1 & 1 & 1 & 1 & 1 \\
0 & 0 & 0 & 0 & 0 & 1 & 1 & 0 & 0 \\
-1 & 0 & 0 & 0 & 1 & 0 & 0 & 0 & 1 \\
0 & 0 & 0 & 1 & 0 & 0 & 0 & -1 & 0 \\
0 & 0 & 0 & 0 & 0 & 1 & -1 & 0 & 0
\end{array}\right]
$$

Because both this triangulation and the solid torus triangulation have a degree one edge, the computation is very similar to that of Example 11.1.

Let $\omega=\left(x_{1} \mu_{1}+y_{1} \lambda_{1}, x_{2} \mu_{2}+y_{2} \lambda_{2}\right)$. Then

$$
\begin{aligned}
I_{\mathcal{T}}(\omega)(q) & =\sum_{k_{2} \in \mathbb{Z}} q^{k_{2}} J_{\Delta}\left(k_{2}-y_{1}, 0,0\right) J_{\Delta}\left(x_{2}, y_{1}, x_{1}+y_{2}\right) J_{\Delta}\left(x_{1}-y_{2},-x_{2}, y_{1}\right) \\
& =J_{\Delta}\left(k_{2}-y_{1}, 0,0\right) J_{\Delta}\left(x_{2}, y_{1}, x_{1}+y_{2}\right) q^{y_{1}} \sum_{k_{2} \in \mathbb{Z}} q^{k_{2}-y_{1}} J_{\Delta}\left(k_{2}-y_{1}, 0,0\right)=0
\end{aligned}
$$

since

$$
\sum_{\ell \in \mathbb{Z}} q^{\ell} J_{\Delta}(\ell, 0,0)=\sum_{\ell \in \mathbb{Z}} I_{\Delta}(-\ell, 0) q^{\ell}=\sum_{\ell \in \mathbb{Z}} I_{\Delta}(0, \ell) q^{\ell}=I(0, q, q)=\frac{\left(q q^{-1} ; q\right)_{\infty}}{(q)_{\infty} ; q}=0 .
$$

11.4. A toroidal example. The 2-tetrahedron ideal triangulation $\mathcal{T}$ with isomorphism signature 'cPcbbbdei' is not 1-efficient. In fact it gives a manifold containing an incompressible torus which splits the manifold in two Seifert fibres pieces $S F S\left[D^{2}(2,1)(3,1)\right]$ and 
$S F S\left[A^{2}(2,1)\right]$. The gluing equation/holonomy coefficients (from SnapPy) are given by:

$$
\left[\begin{array}{cccccc}
1 & 1 & 0 & 1 & 0 & 1 \\
1 & 1 & 2 & 1 & 2 & 1 \\
0 & -1 & 0 & 1 & 0 & 0 \\
0 & 0 & 2 & 0 & 0 & 0
\end{array}\right]
$$

Hence, summing over edge 1 , the 3D-index is given by

$$
I_{\mathcal{T}}(x \mu+y \lambda)=\sum_{k \in \mathbb{Z}} q^{k} J_{\Delta}(k, k-x, 2 y) J_{\Delta}(k+x, 0, k)=\sum_{k \in \mathbb{Z}} I_{\Delta}(x, 2 y-k) I_{\Delta}(-x,-k) .
$$

If $x=0, y=0$ then the above sum $\sum_{k} I_{\Delta}(0,-k)^{2}$ is divergent, as expected by Theorem 5.2 , since each term with $k \leq 0$ has degree 0 .

However for $x \neq 0$ the sum converges, and experimentation suggests the sum simplifies to the following geometric series:

$$
I_{\mathcal{T}}(x \mu+y \lambda)=\frac{(-1)^{x} q^{|x|(|y+x / 2|+1 / 2)}}{\left(1-q^{|x|}\right)}, \text { for } x \neq 0 .
$$

Putting

$$
x \mu+y \lambda=a \mu+(b-a / 2) \lambda=a(\mu-\lambda / 2)+b \lambda=a \bar{\mu}+\beta \bar{\lambda}
$$

we have symmetries $I_{\mathcal{T}}(a \bar{\mu}+b \bar{\lambda})=I_{\mathcal{T}}( \pm a \bar{\mu}+ \pm b \bar{\lambda}$ ) for $a \neq 0$ (by the duality and triality identities) and experimentally it seems that

$$
I_{\mathcal{T}}(a \bar{\mu}+b \bar{\lambda})=\frac{(-1)^{a} q^{a(b+1 / 2)}}{1-q^{a}}, \text { for } a>0, b \geq 0 .
$$

11.5. $\mathbf{m 0 0 9}$. The manifold $X=m 009$ is the first orientable cusped manifold in the SnapPea census with non-peripheral $\mathbb{Z} / 2 \mathbb{Z}$ homology. It has a triangulation using 3 tetrahedra with gluing equation/holonomy coefficients $E_{0}, E_{1}, E_{2}, M, L$ (from SnapPy) given by:

$$
\left[\begin{array}{ccccccccc}
2 & 0 & 0 & 2 & 1 & 0 & 2 & 1 & 0 \\
0 & 2 & 0 & 0 & 0 & 2 & 0 & 0 & 2 \\
0 & 0 & 2 & 0 & 1 & 0 & 0 & 1 & 0 \\
0 & -1 & 1 & 0 & 1 & -1 & 0 & 0 & -1 \\
-1 & 0 & 0 & 1 & 0 & 0 & -2 & 0 & 1
\end{array}\right]
$$

and the tetrahedral solutions $T_{0}, T_{1}, T_{2}$ have coefficients

$$
\left[\begin{array}{lllllllll}
1 & 1 & 1 & 0 & 0 & 0 & 0 & 0 & 0 \\
0 & 0 & 0 & 1 & 1 & 1 & 0 & 0 & 0 \\
0 & 0 & 0 & 0 & 0 & 0 & 1 & 1 & 1
\end{array}\right]
$$

Here,

- $H_{1}(X ; \mathbb{Z}) \cong \mathbb{Z} \times \mathbb{Z} / 2 \mathbb{Z}$

- $H_{2}(X, \partial X ; \mathbb{Z} / 2 \mathbb{Z}) \times H_{1}(\partial X ; \mathbb{Z}) \cong \mathbb{Z} / 2 \mathbb{Z} \times \mathbb{Z}^{2}$ with $\mathbb{Z}^{2}$ generated by the homology classes $\mu, \lambda$ of the "meridian" and "longitude" chosen by SnapPy.

- $\mathcal{K}=\operatorname{Ker}\left(H_{1}(\partial X ; \mathbb{Z}) \rightarrow H_{1}(X ; \mathbb{Z} / 2 \mathbb{Z})\right)$ is spanned by $\mu+\lambda, \mu-\lambda$ 
38 STAVROS GAROUFALIDIS, CRAIG D. HODGSON, NEIL R. HOFFMAN, AND J. HYAM RUBINSTEIN

- The taut angle structure with angles $\alpha=(0, \pi, 0,0, \pi, 0,0, \pi, 0)$ has vanishing peripheral rotational holonomy, so can be used to compute Euler characteristics via $\chi=\sum-\alpha(q) / \pi$.

- $S_{1}=\frac{1}{2} E_{1}=\left[\begin{array}{lllllllll}0 & 1 & 0 & 0 & 0 & 1 & 0 & 0 & 1\end{array}\right]$ has $\left(\left[S_{1}\right]_{2},\left[\partial S_{1}\right]\right)=(\overline{1}, 0) \in \mathbb{Z} / 2 \mathbb{Z} \times \mathbb{Z}^{2}$ and $\chi\left(S_{1}\right)=-1$

- $N(\mathcal{T} ; \mathbb{Z})$ is spanned over $\mathbb{Z}$ by $E_{0}, E_{1}, E_{2}, T_{0}, T_{1}, T_{2}$ and $S_{1}$.

- $M$ has $\left([M]_{2},[\partial M]\right)=(0,2 \mu) \in \mathbb{Z} / 2 \mathbb{Z} \times \mathbb{Z}^{2}$ and $\chi(M)=0$

- $L$ has $\left([L]_{2},[\partial L]\right)=(0,2 \lambda) \in \mathbb{Z} / 2 \mathbb{Z} \times \mathbb{Z}^{2}$ and $\chi(L)=0$

- $S_{2}=\frac{1}{2}\left(M+L+T_{0}-T_{1}\right)=\left[\begin{array}{lllllllll}0 & 0 & 1 & 0 & 0 & -1 & -1 & 0 & 0\end{array}\right]$ has $\left(\left[S_{2}\right]_{2},\left[\partial S_{2}\right]\right)=$ $(0, \mu+\lambda) \in \mathbb{Z} / 2 \mathbb{Z} \times \mathbb{Z}^{2}$ and $\chi\left(S_{2}\right)=0$

- $Q(\mathcal{T} ; \mathbb{Z})$ is spanned over $\mathbb{Z}$ by $E_{0}, E_{1}, E_{2}, T_{0}, T_{1}, T_{2}, S_{1}, M, L$ and $S_{2}$.

To compute the index for the 'even' class $(\overline{0}, 2 x, 2 y) \in \mathbb{Z} / 2 \mathbb{Z} \times \mathbb{Z}^{2}$ we sum over elements of $(\mathbb{E}+\mathbb{T}) / \mathbb{T}$ by taking edge coefficients $\left(0, k_{1}, k_{2}\right)$ with $k_{1}, k_{2} \in \mathbb{Z}$ giving

$$
\begin{aligned}
I^{\text {even }}(2 x \mu+2 y \lambda) & =\sum_{k_{1}, k_{2}} q^{k_{1}+k_{2}} J_{\Delta}\left(k_{1} E_{1}+k_{2} E_{2}+x M+y L\right) \\
& =\sum_{k_{1}, k_{2}} q^{k_{1}+k_{2}} J_{\Delta}\left(-y, 2 k_{1}-x, 2 k_{2}\right) J_{\Delta}\left(y, k_{2}+x, 2 k_{1}-x\right) J_{\Delta}\left(-2 y, k_{2}, 2 k_{1}-x+y\right)
\end{aligned}
$$

e.g

$$
I^{\text {even }}(0,0)=1-q-q^{2}+6 q^{3}+9 q^{4}+12 q^{5}-5 q^{6}-34 q^{7}-79 q^{8}-118 q^{9}-118 q^{10}+\ldots
$$

To compute the index for the 'odd' class $(\overline{1}, 2 x, 2 y) \in \mathbb{Z} / 2 \mathbb{Z} \times \mathbb{Z}^{2}$ we sum over elements of the coset $\left(S_{1}+\mathbb{E}+\mathbb{T}\right) / \mathbb{T}$ by taking edge coefficients $\left(0, \frac{1}{2}+k_{1}, k_{2}\right), k_{1}, k_{2} \in \mathbb{Z}$ giving

$$
\begin{aligned}
I^{\text {odd }}(2 x \mu+2 y \lambda)=\sum_{k_{1}, k_{2}}\left(-q^{1 / 2}\right) q^{k_{1}+k_{2}} J\left(S_{1}+k_{1} E_{1}+k_{2} E_{2}+x M+y L\right) \\
=\sum_{k_{1}, k_{2}}-q^{1 / 2+k_{1}+k_{2}} J_{\Delta}\left(-y, 2 k_{1}+1-x, 2 k_{2}\right) J_{\Delta}\left(y, k_{2}+x, 2 k_{1}+1-x\right) \\
\times J_{\Delta}\left(-2 y, k_{2}, 2 k_{1}+1-x+y\right)
\end{aligned}
$$

e.g.

$I^{\text {odd }}(0,0)=-q^{1 / 2}-2 q^{3 / 2}+2 q^{5 / 2}+8 q^{7 / 2}+11 q^{9 / 2}+6 q^{11 / 2}-17 q^{13 / 2}-57 q^{15 / 2}-100 q^{17 / 2}-124 q^{19 / 2}+\ldots$

For the class $(\overline{0}, 1,1) \in \mathbb{Z} / 2 \mathbb{Z} \times \mathbb{Z}^{2}$ we take edge coefficients $\left(0, k_{1}, k_{2}\right)$ with $k_{1}, k_{2} \in \mathbb{Z}$ giving

$$
\begin{aligned}
I^{\text {even }}(\mu+\lambda) & =\sum_{k_{1}, k_{2}} q^{k_{1}+k_{2}} J\left(S_{2}+k_{1} E_{1}+k_{2} E_{2}\right) \\
& =\sum_{k_{1}, k_{2}} q^{k_{1}+k_{2}} J_{\Delta}\left(0,2 k_{1}, 2 k_{2}+1\right) J_{\Delta}\left(0, k_{2}, 2 k_{1}-1\right) J_{\Delta}\left(-1, k_{2}, 2 k_{1}\right) \\
& =-q+4 q^{3}+7 q^{4}+6 q^{5}-7 q^{6}-32 q^{7}-65 q^{8}-89 q^{9}-81 q^{10}+\ldots
\end{aligned}
$$




\section{Notes on Connectedness of the 1-Efficient Pachner Graph}

In this section we study the Pachner graph for a compact orientable 3-manifold $M$ with non-empty boundary consisting of tori. This is a graph where each vertex corresponds to an ideal triangulation (up to the equivalence of relabelling) of the manifold $M$, with an edge connecting two vertices if their corresponding triangulations can be obtained from one another via 2-3 or 3-2 Pachner moves (as shown in Figures 11 and 12.) We give some general results, then describe a census of all ideal triangulations with at most 6 tetrahedra which provides a number of examples with interesting properties.

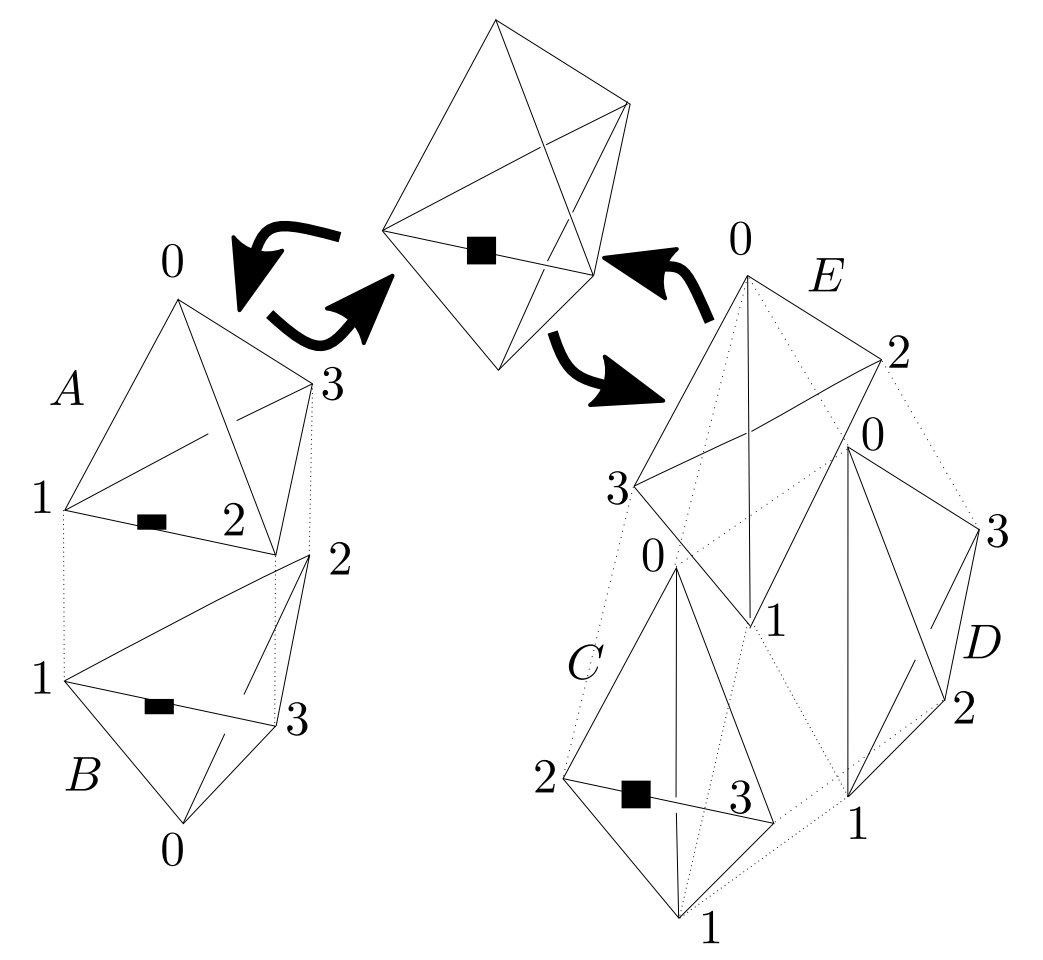

FigurE 11. A 2-3 move on labelled tetrahedra. As the black square indicates, edges $A 12$ and $B 13$ are identified with $C 23$

Throughout the section, we will use Burton's isomorphism signature notation [Bur11] to identify triangulations. Interested readers can replicate the results of this section by inputing these signatures into Regina to construct the relevant triangulations. Readers new to this concept will find it helpful to know that if the first letter of the signature (of those used in this paper) is the $n$-th letter of the alphabet, then the corresponding triangulation is comprised of $n-1$ tetrahedra.

As mentioned in the introduction, the the Pachner graph of a 3-manifold with non-empty boundary is connected, and so it is interesting to consider basic properties of subgraphs of this graph: number of vertices, connectedness, etc. For example, we say the geometric Pachner graph is the subgraph of "geometric" triangulations such that Thurston's gluing equations have a solution where each tetrahedral shape has positive imaginary part. 


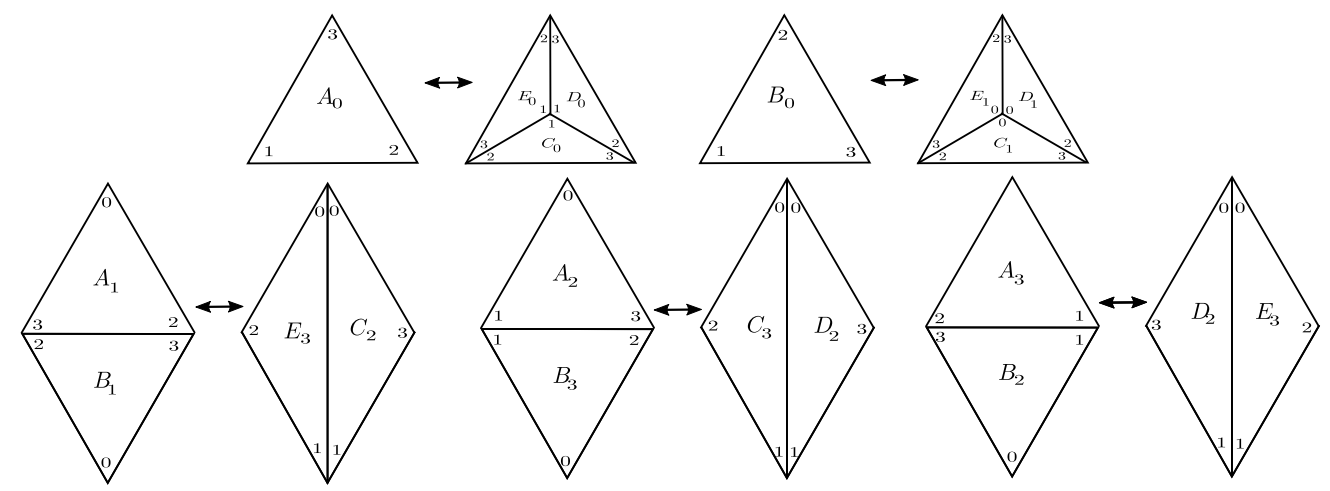

FiguRE 12. The effect of the 2-3 move on the boundary triangles.

It has been recently shown that the figure eight knot complement admits infinitely many geometric triangulations, however the geometric subgraph of the Pachner graph is disconnected (see [DD, Theorem 1.1 and Remark 3.3]).

One can also define the 1-efficient Pachner graph as the subgraph of the Pachner graph containing only 1-efficient triangulations and the edges between them.

Before discussing the 1-efficient Pachner graph, we mention that our notation (used here and in the accompanying code) for Seifert fibred spaces with non-empty boundary, is consistent with Hatcher [Hat]. Namely, a Seifert fibre space over the surface $F$ with exceptional fibres given by the parameters $\left\{\left(a_{i}, b_{i}\right)\right\}_{i=1}^{k}$ (with $a_{i}>b_{i}>0$ if $F$ is punctured) will be denoted by $S F S\left[F\left(a_{1}, b_{1}\right) \ldots\left(a_{k}, b_{k}\right)\right]$.

First, we point out that for many 3-manifolds the 1-efficient Pachner graph is infinite. Although this is presumably known to the experts, we include it for the sake of completeness.

Proposition 12.1. Let $M$ be an cusped irreducible, atoroidal orientable 3-manifold other then the solid torus $S^{1} \times D^{2}$. Then $M$ admits infinitely many 1-efficient triangulations.

The key idea for this proof is to exhibit ideal triangulations that support a taut angle structure, i.e. a semi-angle structure where all angles are either 0 or $\pi$. (A semi-angle structure is an assignment of non-negative angles to the edges in the ideal tetrahedra, so that the angle sum at each ideal vertex in such a tetrahedron is $\pi$.) Also, in [Lac00, Theorem 1], Lackenby shows that an-annular cusped manifolds admit taut ideal triangulations, which give such a taut angle structure. Given the hypotheses of the Proposition, we point out that an-annular is shorthand for Seifert fibre spaces over the disk over than $S^{1} \times D^{2}$ or $S F S[D(2,1)(2,1)]$, and hyperbolic manifolds.

Proof. If $M$ is hyperbolic or a Seifert fibre space over the disk (other than $S^{1} \times D^{2}$ or $S F S[D(2,1)(2,1)])$, then by [Lac00, Theorem 1], $M$ admits a taut ideal triangulation, say with $n$ tetrahedra. In this triangulation, about any edge we can find two faces of the triangulation incident to that edge which (in an open neighbourhood of the edge) separate the two angles labelled by $\pi$. Performing a 0-2 move along these two faces produces a new triangulation with $n+2$ tetrahedra. To see that this triangulation supports a taut angle structure, label each edge in the new edge class of degree 2 by $\pi$, the opposite edges in the 
tetrahedron by $\pi$, and the remaining edges 0 . As this is a semi-angle structure, this new triangulation is 1-efficient since $M$ is atoroidal (see [KR05, Theorem 2.6]).

If $M$ is Seifert fibred over an annulus with exactly one exceptional fibre, then $M$ is fibred over the circle with fibre a surface of negative Euler characteristic. Therefore, $M$ admits a layered triangulation obtained from expressing the monodromy of its fibration in terms of edge flips on an ideal triangulation of the fibre (see for example [Lac00, Section 2]). Using this ideal layered triangulation, we again obtain a taut angle structure and complete the proof in the same manner as above.

The following remark uses a dual notion to 1-efficiency, introduced by Garoufalidis [Gar15], to obtain a similar result. Let $\mathcal{T}$ be an ideal triangulation with $n$ tetrahedra and $\Lambda=\left\{\lambda_{i}\right\}_{i=1}^{3 n}$ a set of $3^{n}$ generalised angle structures. Then $\Lambda$ is a index structure on $\mathcal{T}$ if for each $Q=$ $\left(Q_{1}, \ldots, Q_{n}\right)$ where $Q_{j}$ is a choice of quadrilateral type in tetrahedron $j$, there exist $\lambda_{i}$ such that for all $j$ the edges opposite $Q_{j}$ in $\mathcal{T}$ have positive angles in $\lambda_{i}$. This is an obstruction to having embedded surfaces of non-negative Euler characteristic, and in fact it is known to be equivalent to $\mathcal{T}$ being 1 -efficient by [GHRS15, Theorem 1.2].

Remark 12.2. An ideal triangulation of a solid torus will not admit a taut angle structure (by [KR05], the existence of an embedded generalised normal torus in a solid torus is an obstruction to existence of a taut angle structure). Nevertheless, an analogous method can be used to obtain infinitely many 1-efficient triangulations of the solid torus. For example, the triangulation 'dLQacccbnbb' has a gluing matrix:

$$
\left(\begin{array}{lllllllll}
1 & 0 & 0 & 0 & 0 & 0 & 0 & 0 & 0 \\
1 & 2 & 2 & 1 & 2 & 2 & 1 & 2 & 2 \\
0 & 0 & 0 & 1 & 0 & 0 & 1 & 0 & 0
\end{array}\right)
$$

and so $\left\{\left(2 \pi, \beta_{0},-\pi-\beta_{0}, \pi, \beta_{1},-\beta_{1}, \pi, \beta_{2},-\beta_{2}\right) \mid \beta_{0}, \beta_{1}, \beta_{2} \in \mathbb{R}\right\}$ is a subset of the generalised angle structures on 'dLQacccbnbb'. Furthermore, one has the freedom to adjust these parameters to find an index structure. In fact, after performing a 0-2 move along the two faces incident to the degree two edge, we see that a new index structure is obtained since we can obtain new generalised angle structures by just introducing two new tetrahedra subject to same constraint as the last two tetrahedra above, i.e. the angles are $\left(\pi, \beta_{j},-\beta_{j}\right)$. Furthermore, one can then perform 0-2 moves along the faces incident to any degree 2 edge in the resulting triangulations to obtain more 1-efficient triangulations, and so by an inductive argument there are infinitely many 1-efficient triangulations of the solid torus.

12.1. A census of ideal triangulations. These examples were obtained by first compiling a list of all ideal triangulations with at most six tetrahedra using Regina's tricensus function. Then it was determined if these triangulations were solid tori. Of the remaining triangulations, we then (i) determined their irreducibility and (ii) checked for incompressible tori and Klein bottles. The first condition was checked using a light adaptation of Regina's isThreeSphere function and the second determined using Regina's isSolidTorus and a new function isT2xI (see [Bur]). The latter function is similar to Haraway's algorithm [Har, Corollary 15], but instead of using an analysis of spun normal annuli, it relies on the fact that if $M$ is a 2-cusped manifold with three $S^{1} \times D^{2}$ surgeries along slopes $\left\{\gamma_{1}, \gamma_{2}, \gamma_{3}\right\}$, then $M$ is homeomorphic to $T^{2} \times I$ the Berge manifold [Ber91, Gab89], or is Seifert fibred over the 
annulus with one exceptional fibre. However, all of the $S^{1} \times D^{2}$ fillings of the Berge manifold are pairwise distance one from each other, and so if a manifold admits $S^{1} \times D^{2}$ fillings along slopes $\left\{\frac{1}{0},-\frac{1}{1}, \frac{2}{1}\right\}$ then it must be $T^{2} \times I$ or Seifert fibred over the annulus. The latter can be ruled out since at least one of the fillings $\left\{\frac{1}{0},-\frac{1}{1}, \frac{2}{1}\right\}$ will result in a Seifert fibred space over the disk with two (non-trivial) exceptional fibres.

This allows for a decomposition into prime and atoroidal pieces. Those pieces were then classified by searching the Pachner graph of the corresponding triangulations and simplifying the triangulation to that of either a Snappy OrientableCuspCensus triangulation (using SnapPy's identify function) or until it was equivalent via Pachner moves to a known triangulation of a lens space (in the case of some prime summands) or a cusped Seifert fibered space (using a dictionary of triangulations also included with the code). While a larger library of closed triangulations would be needed for more complicated prime and JSJ decompositions, this was sufficient for our purposes.

After this coarse classification, the triangulations were then analysed for 1-efficiency. The results of this census are summarised in Tables 1 and 2 below; the code used is available as an ancillary file to the arxiv version of this paper.

\begin{tabular}{|c|c|c|c|c|c|c|c|c||c|c|}
\hline $\mathrm{n}$ & total & $S^{1} \times D^{2}$ & $T^{2} \times I$ & $P \times S^{1}$ & Red. & Tor. & SFS & Hyp. & Taut & 1-efficient \\
\hline 2 & 10 & 3 & 0 & 0 & 0 & 1 & 4 & 2 & 7 & 9 \\
\hline 3 & 129 & 65 & 1 & 0 & 0 & 15 & 36 & 12 & 53 & 102 \\
\hline 4 & 1852 & 917 & 11 & 0 & 107 & 188 & 491 & 138 & 441 & 1082 \\
\hline 5 & 26909 & 14324 & 197 & 1 & 2533 & 2164 & 6344 & 1346 & 3310 & 12130 \\
\hline 6 & 414946 & 219080 & 2981 & 32 & 58508 & 29451 & 89933 & 14961 & 134538 & 28405 \\
\hline
\end{tabular}

TABLE 1. This table provides information on the various types of (orientable) ideal triangulations that can be built from $n=2,3,4,5,6$ tetrahedra. Total is the total number of triangulations for each value of $n, S^{1} \times D^{2}$ is the number of solid tori, similarly for $T^{2} \times I$ and $P \times S^{1}$. Red. counts the number of reducible manifolds and Tor. counts the number of toroidal manifolds which are not homeomorphic to $S F S[D(2,1)(2,1)]$. Instead $S F S[D(2,1)(2,1)]$ is counted in SFS which record the number of SFS over the disk or annulus with two or one exceptional fibres respectively. Finally, Hyp. counts the number of hyperbolic manifolds observed. Note that in each case, this computation is rigorous, in the sense that the triangulation was connected to a known triangulation via Pachner moves. Finally, Taut records the number of triangulations admitting a taut angle structure and 1-efficient records the number of 1-efficient triangulations.

12.2. Two tetrahedron ideal triangulations. Up to relabelling, there are 10 triangulations of orientable cusped 3-manifolds that decompose into two tetrahedra, and these 10 triangulations correspond to seven manifolds up to homeomorphism. The relevant data are presented in Table 3. 


\begin{tabular}{|c|c|c|c|c|c|c|c|}
\hline $\mathrm{n}$ & Hyp. & Geom. & Semi-Geom. & Strict Ang. Struct. & Semi-Ang. & Taut & 1-efficient \\
\hline 2 & 2 & 2 & 2 & 2 & 2 & 2 & 2 \\
\hline 3 & 12 & 12 & 12 & 12 & 12 & 12 & 12 \\
\hline 4 & 138 & 110 & 121 & 119 & 134 & 134 & 134 \\
\hline 5 & 1346 & 670 & 798 & 801 & 1165 & 1118 & 1190 \\
\hline 6 & 14961 & 3857 & 4923 & 5221 & 10908 & 9927 & 11512 \\
\hline
\end{tabular}

TABLE 2. This table provides data on the ideal triangulations of hyperbolic manifolds in the census. Hyp. gives the total number of hyperbolic manifolds. Geom. gives the number of triangulations which SnapPy found an approximate hyperbolic structure and Semi-Geom. records the number of triangulations which SnapPy found an approximate hyperbolic structure possibly with flat (and non-degenerate) tetrahedra. Strict. Ang. Struct. records the number of triangulations admitting a strict angle structure, while Semi-Ang. records the number of triangulations admitting a semi-angle structure. Finally, Taut records the number of triangulations admitting a Taut structure and 1-efficient records the number of 1-efficient triangulations of hyperbolic manifolds.

\begin{tabular}{|c|c|c|}
\hline signature & name & 1-efficient \\
\hline \hline cMcabbgds & $S^{1} \times D^{2}$ & Yes \\
\hline cMcabbgij & $S^{1} \times D^{2}$ & Yes \\
\hline cMcabbgik & $S^{1} \times D^{2}$ & Yes \\
\hline \hline cPcbbbalm & $S F S\left[D^{2}(3,1)(3,1)\right]$ & Yes \\
\hline \hline cPcbbbali & $S F S\left[D^{2}(3,1)(3,2)\right]$ & Yes \\
\hline \hline cPcbbbadh & $S F S\left[D^{2}(2,1)(3,1)\right]$ & Yes \\
\hline cPcbbbadu & $S F S\left[D^{2}(2,1)(3,1)\right]$ & Yes \\
\hline \hline cPcbbbdxm & Figure 8 sister $\mathbf{( m 0 0 3 )}$ & Yes \\
\hline \hline cPcbbbiht & Figure $\mathbf{8}(\mathbf{m 0 0 4})$ & Yes \\
\hline \hline cPcbbbdei & $S F S\left[D^{2}(2,1)(3,1)\right] \cup S F S\left[A^{2}(2,1)\right]$ & No \\
\hline
\end{tabular}

TABLE 3. The complete census of ideal triangulations with two tetrahedra. The homeomorphism descriptions come from an appeal to the surgery description given by Martelli and Petronio [MP06], (which the diligent reader can verify using a tangle computation).

\section{Some Pachner Paths.}

To provide a certificate of a path in the Pachner graph we list the vertices and also provide the face consumed by a 2-3 move or the edge consumed by a 3-2 move. In the first case, we take the index of the face in the labelled triangulation determined by the isomorphism signature. In the second, we take $-i n d e x-1$ of the edge (the minus sign indicates that an edge is being consumed and shifting by -1 removes the ambiguity of -0 and +0 that would arise if we were required to perform a 3-2 along edge 0 ). 
Example 12.3. Solid Torus. The (open) solid torus has 3 minimal ideal triangulations with 2 tetrahedra. The paths shown in Table 4 are 1-efficient paths in the Pachner graph connecting these minimal triangulations.

\begin{tabular}{|c|c||c|c||c|c|}
\hline Path 1 & & Path 2 & Path 3 & \\
\hline isoSig & $2-3$ move & isoSig & $2-3$ move & isoSig & $2-3$ move \\
\hline 'cMcabbgds' & 0 & 'cMcabbgds' & 0 & 'cMcabbgij' & 0 \\
\hline 'dLQbcccaego' & 0 & 'dLQbcccaego' & 4 & 'dLQbcccahgc' & 5 \\
\hline 'eLPkbcdddhgcgj' & -3 & 'eLPkbcdddhcgcf' & -2 & 'eLAkbccddaegtr' & -3 \\
\hline 'dLQbcccahgc' & -3 & 'dLQacccjrgr' & 2 & 'dLQbcbcaekv' & 2 \\
\hline 'cMcabbgij' & 'end' & 'eLPkbcdddhcgbf' & -1 & 'eLAkbccddaegtn' & -4 \\
\hline- & - & 'dLQbcccahgo' & -3 & 'dLQbcccahgo' & -3 \\
\hline- & - & 'cMcabbgik' & 'end' & 'cMcabbgik' & 'end' \\
\hline
\end{tabular}

TABLE 4. Some 1-efficient paths for triangulations of the solid torus

Remark 12.4. This analysis shows that the 1-efficient Pachner graph (of ideal triangulations) of the solid torus is not connected. Specifically, we can say the following: Both 'gLLAQbecefefaaopaaj' and 'gLLAQbecefefaaopaan' are six tetrahedral triangulations of the solid torus that 1) have no degree 3 edges and 2) each 2-3 move along a face in either triangulation results in a not 1-efficient triangulation. However, we can still relate the 3D-index to the 3D-index of the triangulations of the solid torus in Table 3. In fact, both of these six tetrahedral triangulations have (three) degree two edges. After performing a 2-0 move on any degree two edge of one of these triangulations, the resulting triangulation is 1-efficient and connected to the triangulation 'cMcabbgij' via a path of 1 -efficient triangulations. Therefore, the 3D-index is consistent with the two tetrahedral triangulation of an ideal solid torus 'cMcabbgij' by [GHRS15, Theorem 5.1].

Example 12.5. Trefoil Complement. The trefoil complement $\left(\operatorname{SFS}\left[D^{2}(2,1)(3,1)\right]\right)$ has two minimal triangulations that are connected along a path of length 6 (see Figure 13 and Table 5) and a 1-efficient path of length 12 (see Figure 13 and Table 6).

12.3. Angle Structures, 1-efficiency and Pachner moves. A natural question is how are various properties of triangulations related by Pachner moves. Throughout this section, $\mathcal{T}_{n}$ will be a triangulation with $n$ tetrahedra, $f$ will be a face in this triangulation that identifies two distinct tetrahedra $t_{f, 1}$ and $t_{f, 2}$, and $\mathcal{T}_{n+1, f}$ will be the result of performing a 2-3 move along the face $f$. If we call the pairs of edges incident to $f$ in the bi-pyramid defined by $t_{f, 1}$ and $t_{f, 2}$, the belt of $f$, then we can make the following observation that is wellknown to the experts. We include it because it promotes a useful mentality and compares favourably to our discussion of 1-efficiency and Pachner moves.

Proposition 12.6. Let $\mathcal{T}_{n}$ be a triangulation with $n$ tetrahedra. With all notation as above, $\mathcal{T}_{n+1, f}$ admits a strict angle structure if and only if the set of angle structures contains a strict angle structure (resp. semi-angle structure) such that each of the three pairs of edges along the belt of $f$ have a sum in $(0, \pi)$ (resp. $[0, \pi])$. 


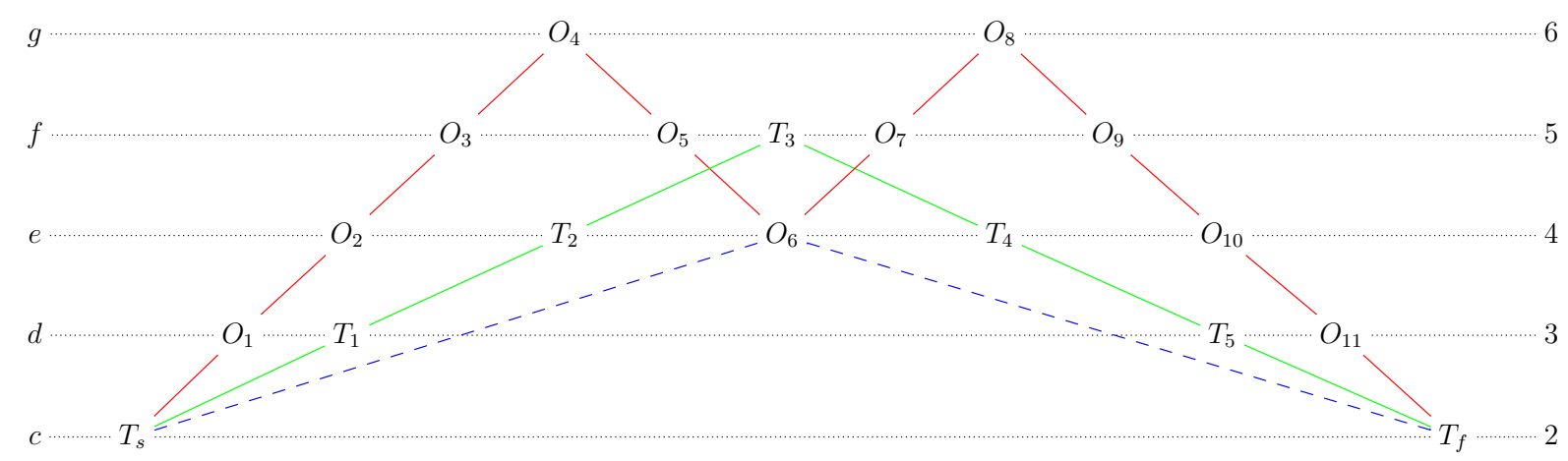

FIGURE 13. A shortest Pachner path between minimal triangulations of the trefoil (green) and a longer 1-efficient path (red). Note, the 1-efficient path is a shortest path through 1 -efficient triangulations using $2-3 / 3-2$ moves. If 0 $2 / 2-0$, moves are allowed then the red and green paths shorten to $\left\{T_{s}, O_{6}, T_{f}\right\}$ (blue dashed). Also, a computation in Regina shows all triangulations on the green path except $T_{s}$ and $T_{f}$ are not 1-efficient.

\begin{tabular}{|c|c|c|}
\hline Figure name & isoSig & move \\
\hline$T_{s}$ & 'cPcbbbadh' & 2 \\
\hline$T_{1}$ & 'dLQacccbgjs' & 1 \\
\hline$T_{2}$ & 'eLPkbcdddacrnn' & 4 \\
\hline$T_{3}$ & 'fvPQccdedeeccrbfb' & -1 \\
\hline$T_{4}$ & 'eLPkbcdddackjj' & -2 \\
\hline$T_{5}$ & 'dLQaccobgbk' & -3 \\
\hline$T_{f}$ & 'cPcbbbadu' & 'end' \\
\hline
\end{tabular}

TABLE 5. A shortest Pachner path between the two minimal triangulations of the trefoil complement.

Proof. We only need to consider the tetrahedra in the bi-pyramid as the other angles will be unaffected by a 2-3 move.

Considering Figure 12, this problem reduces to a problem in the Euclidean plane: namely showing that the triangles incident to the vertices at the top and bottom of the bi-pyramid each split into 3 triangles with angles in the desired range. However, the angles around the vertex are all in $(0, \pi)$ (resp. $[0, \pi])$, so the vertex is embedded in the interior (resp. interior or a side).

The other direction is obvious as an angle structure on $\mathcal{T}_{n+1, f}$ implies that all angles along the edges of the bi-pyramid are positive (resp. non-negative).

12.4. Connected components of normal surfaces. In Figure 11, there are two bipyramids related by a 2-3 move, one with two tetrahedra which we call $\mathcal{B}_{2}$ and one with three tetrahedra which we call $\mathcal{B}_{3}$. We will also think of these as subsets of two triangulations related by a $2-3$ move, $\mathcal{T}_{n}$ and $\mathcal{T}_{n+1, f}$ where $f$ is face identified in $\mathcal{B}_{2}$. In this discussion, we assume that the exterior faces of $\mathcal{B}_{2}$ are not identified. However, if the exterior faces of $\mathcal{B}_{2}$ 
46 STAVROS GAROUFALIDIS, CRAIG D. HODGSON, NEIL R. HOFFMAN, AND J. HYAM RUBINSTEIN

\begin{tabular}{|c|c|c|}
\hline Figure name & isoSig & move \\
\hline$T_{s}$ & 'cPcbbbadh' & 1 \\
\hline$O_{1}$ & 'dLQacccjgjb' & 5 \\
\hline$O_{2}$ & 'eLAkbbcdddugaj' & 2 \\
\hline$O_{3}$ & 'fLLQcccddeeabvnln' & 5 \\
\hline$O_{4}$ & 'gLvQQadfeeffjatxcfj' & -5 \\
\hline$O_{5}$ & 'fLAPcacceejgjffc' & -3 \\
\hline$O_{6}$ & 'eLMkbbdddadiih' & 0 \\
\hline$O_{7}$ & 'fLAPcacceeejgjcrc' & 6 \\
\hline$O_{8}$ & 'gLvQQadfeeffjaaxcfj' & -4 \\
\hline$O_{9}$ & 'fLLQcccddeeabvrln' & -4 \\
\hline$O_{10}$ & 'eLAkbbcdddurar' & -2 \\
\hline$O_{11}$ & 'dLQacccjgjs' & -3 \\
\hline$T_{f}$ & 'cPcbbbadu' & 'end' \\
\hline
\end{tabular}

TABLE 6. A shortest one-efficient Pachner path between the two minimal triangulations of the trefoil complement.

are identified (and therefore $\mathcal{B}_{3}$ ), then some of these pieces might not appear as subsets of embedded normal surfaces.

If $S_{n}$ is a normal surface of $\mathcal{T}_{n}$ then each piece will have at most one normal arc in face $f$. Using the notation from Section 3.1 and Figure 1, we thus obtain a census of connected components $S_{n} \cap \mathcal{B}_{2}$ as follows:

(1) $t_{A 0}, t_{B 1}$;

(2) $\left(t_{A 1}+t_{B 1}\right),\left(t_{A 2}+t_{B 3}\right),\left(t_{A 3}+t_{B 2}\right)$;

(3) $\left(q_{A 01: 23}+t_{B 1}\right),\left(q_{A 02: 13}+t_{B 3}\right),\left(q_{A 03: 12}+t_{B 2}\right),\left(q_{B 01: 23}+t_{A 1}\right),\left(q_{B 02: 13}+t_{A 3}\right)$, and $\left(q_{B 03: 12}+t_{A 2}\right)$

(4) $\left(q_{A 01: 23}+q_{B 01: 23}\right),\left(q_{A 02: 13}+q_{B 03: 12}\right),\left(q_{A 03: 12}+q_{B 02: 13}\right)$.

If $S_{n+1}$ is a normal surface of $\mathcal{T}_{n+1, f}$, we can obtain an analogous result. Namely, the observation that each connected component of $S_{n+1} \cap \mathcal{B}_{3}$ intersects an interior face of $\mathcal{B}_{3}$ in at most one normal arc, yields the following list:

(1) $\left(t_{C 0}+t_{D 0}+t_{E 0}\right),\left(t_{C 1}+t_{D 1}+t_{E 1}\right)$;

(2) $\left(t_{C 2}+t_{E 3}\right),\left(t_{D 2}+t_{C 3}\right),\left(t_{D 3}+t_{E 2}\right)$;

(3) $\left(t_{D 0}+q_{C 02: 13}+q_{E 03: 12}\right),\left(t_{E 0}+q_{D 02: 13}+q_{C 03: 12}\right),\left(t_{C 0}+q_{E 02: 13}+q_{D 03: 12}\right),\left(q_{D 01: 23}+\right.$ $\left.t_{C 3}+t_{E 2}\right),\left(t_{E 1}+q_{D 03: 12}+q_{C 02: 13}\right),\left(t_{C 1}+q_{E 03: 12}+q_{D 02: 13}\right)$

(4) $\left(q_{D 01: 23}+t_{C 3}+t_{E 2}\right),\left(q_{E 01: 23}+t_{D 3}+t_{C 2}\right),\left(q_{C 01: 23}+t_{E 3}+t_{D 2}\right)$;

(5) $\left(q_{C 01: 23}+q_{D 01: 23}+t_{E 2}+t_{E 3}\right),\left(q_{D 01: 23}+q_{E 01: 23}+t_{C 2}+t_{C 3}\right),\left(q_{E 01: 23}+q_{C 01: 23}+t_{D 2}+t_{D 3}\right)$;

(6) $\left(q_{C 01: 23}+q_{D 01: 23}+q_{E 01: 23}\right)$.

Examples of the normal surface pieces of type 3) and 4) are given in Figures 14 and 15.

Examples of the normal surface pieces of type 5) and 6) are given in Figures 16 and 17.

The following proposition allows us relate the pieces in both list above via the 2-3 move that transforms $\mathcal{B}_{2}$ to $\mathcal{B}_{3}$. 


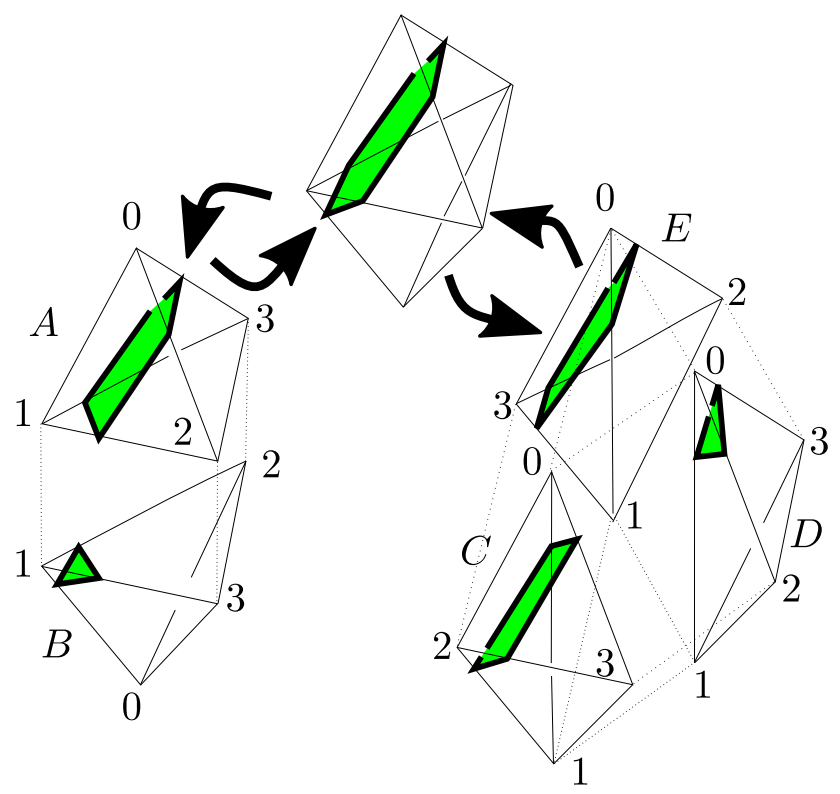

FIGURE 14. The effect of a 2-3 move on a normal disk comprised of a triangle and quad.

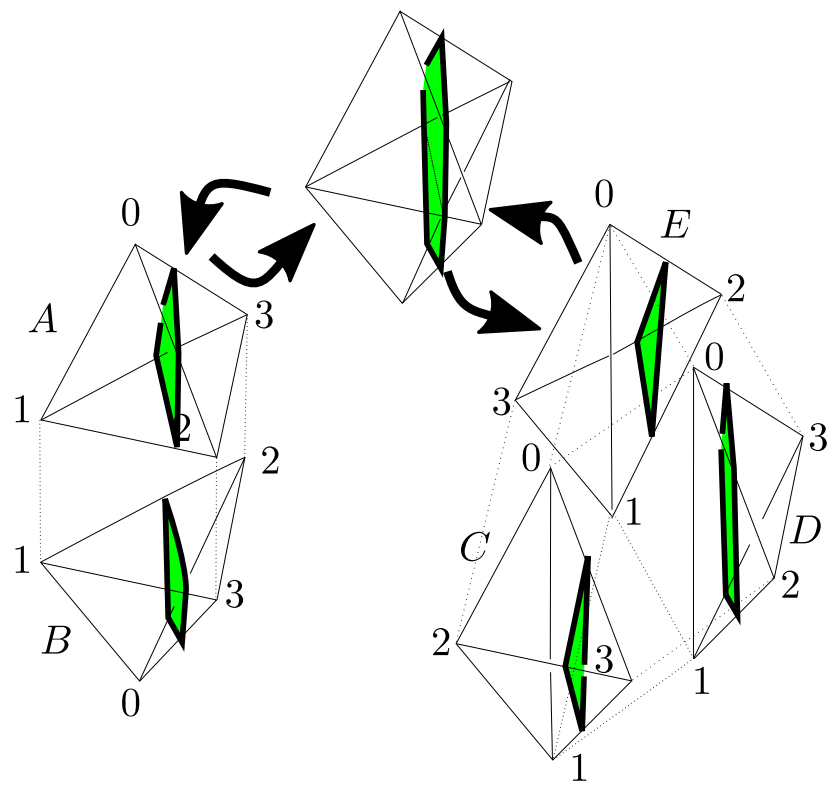

Figure 15. The effect of a 2-3 move on a normal disk comprised of two quads. 


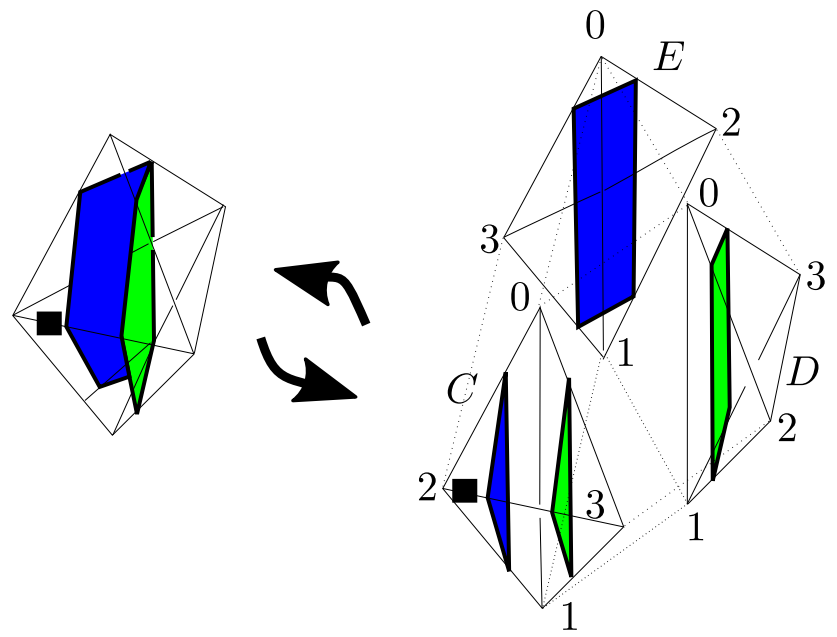

FigurE 16. An example of a normal surface piece of $\mathcal{B}_{3}$.
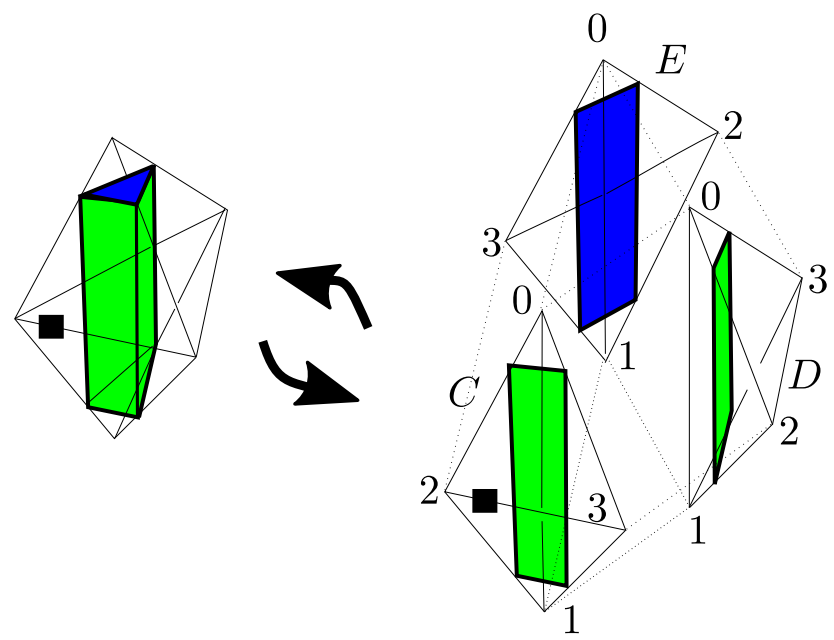

FiguRE 17. A second example of a normal surface piece of $\mathcal{B}_{3}$.

Proposition 12.7. Let $\mathcal{T}_{n}$ and $\mathcal{T}_{n+1, f}$ be the triangulations defined above. For each normal surface $S_{n}$ of $\mathcal{T}_{n}$ there exists a normal surface $S_{n+1}$ of $\mathcal{T}_{n+1, f}$ obtained by dividing up the normal discs of $S_{n}$ which intersect $\mathcal{B}_{2}$. In particular, if $\mathcal{T}_{n+1, f}$ is 1-efficient, then $\mathcal{T}_{n}$ is 1-efficient.

Proof. Following Figure 11, we can consider the images of $S_{n} \cap \mathcal{B}_{2}$ under the 2-3 move:

(1) $t_{A 0}=t_{C 0}+t_{D 0}+t_{E 0}, t_{B 0}=t_{C 1}+t_{D 1}+t_{E 1}$

(2) $t_{A 1}+t_{B 1}=t_{C 2}+t_{E 3}, t_{A 2}+t_{B 3}=t_{D 2}+t_{C 3}, t_{A 3}+t_{B 2}=t_{E 2}+t_{D 3}$

(3) $q_{A 01: 23}+t_{B 1}=t_{D 0}+q_{C 02: 13}+q_{E 03: 12}, q_{A 02: 13}+t_{B 3}=t_{E 0}+q_{D 02: 13}+q_{C 03: 12}, q_{A 03: 12}+$ $t_{B 2}=t_{C 0}+q_{E 02: 13}+q_{D 03: 12}, q_{B 01: 23}+t_{A 1}=t_{D 1}+q_{C 03: 12}+q_{E 02: 13}, q_{B 02: 13}+t_{A 3}=$ $t_{E 1}+q_{D 03: 12}+q_{C 02: 13}, q_{B 03: 12}+t_{A 2}=t_{C 1}+q_{E 03: 12}+q_{D 02: 13}$,

(4) $q_{A 01: 23}+q_{B 01: 23}=q_{D 01: 23}+t_{C 3}+t_{E 2}, q_{A 02: 13}+q_{B 03: 12}=q_{E 01: 23}+t_{D 3}+t_{C 2}, q_{A 03: 12}+$ $q_{B 02: 13}=q_{C 01: 23}+t_{E 3}+t_{D 2}$. 
Relations (1) and (2) follow from Figure 12.

Note that $q_{A 01: 23}+t_{B 1}=t_{D 0}+q_{C 02: 13}+q_{E 03: 12}$ follows from Figure 14, and the remaining two relations can be obtained by an appropriate rotation (and relabelling) of that figure.

Finally, $q_{A 01: 23}+q_{B 01: 23}=q_{D 01: 23}+t_{C 3}+t_{E 2}$ follows from Figure 15, and again the remaining two relations can be obtained by an appropriate rotation (and relabelling) of that figure.

Thus, for any normal surface of $S_{n}$, we can map the normal surface pieces locally to build a normal surface with the same Euler characteristic in $\mathcal{T}_{n+1, f}$. Restricting this observation to the case of embedded normal surfaces with non-negative Euler characteristics shows if $\mathcal{T}_{n+1, f}$ is 1-efficient, then $\mathcal{T}_{n}$ must be as well.

We point out that the Luo-Tilmann's definition of Euler characteristic on quadrilateral discs in the presence of a generalised angle structure (compare [LT08, Lemma 15] in the case that the curvature is 0) provides a useful mnemonic for keeping track of relations of type (3) and (4). Namely, these relations are just the angle sums which occur after a 2-3 move, e.g. edge $A 01$ decomposes into edges $C 02$ and E03 and edges $A 23$ and B23 form D23.

Proposition 12.8. Let $\mathcal{T}_{n}$ and $\mathcal{T}_{n+1, f}$ be the triangulations defined above, such that the exterior faces of $\mathcal{B}_{2}$ are not identified. If $\mathcal{T}_{n}$ is 1-efficient and $\mathcal{T}_{n+1, f}$ is not 1-efficient, then $\mathcal{T}_{n+1, f}$ exhibits an embedded normal surface with exactly two of the three quad types parallel to the degree three edge created by the 2-3 move.

Proof. If $\mathcal{T}_{n}$ is 1-efficient and $\mathcal{T}_{n+1, f}$ is not 1-efficient, then there must be an embedded normal surface $S_{n+1}$ in $\mathcal{T}_{n+1, f}$ which intersects $\mathcal{B}_{3}$. If this intersection is only triangular discs then $S_{n+1}$ would descend to an embedded normal surface in $\mathcal{T}_{n}$, and so $S_{n+1} \cap \mathcal{B}_{3}$ must contain at least one connected component with at least one quad. However, the case analysis in Proposition 12.7 of the normal discs in $\mathcal{B}_{3}$ shows that each connected component $S_{n+1} \cap \mathcal{B}_{3}$ is in the image of an embedded normal disk of $\mathcal{B}_{2}$ save four: $q_{C 01: 23}+q_{D 01: 23}+t_{E 2}+t_{E 3}$, $q_{D 01: 23}+q_{E 01: 23}+t_{C 2}+t_{C 3}, q_{E 01: 23}+q_{C 01: 23}+t_{D 2}+t_{D 3}$, and $\left(q_{C 01: 23}+q_{D 01: 23}+q_{E 01: 23}\right)$.

However, if the last piece is part of $S_{n+1} \cap \mathcal{B}_{3}$, then there exists a collection of embedded normal spheres $\hat{S}_{n+1}$ of $\mathcal{T}_{n+1, f}$ formed by cutting out all copies of this annulus and capping off with triangles. If none of these spheres have pieces of the form: $q_{C 01: 23}+q_{D 01: 23}+t_{E 2}+t_{E 3}$, $q_{D 01: 23}+q_{E 01: 23}+t_{C 2}+t_{C 3}$, and $q_{E 01: 23}+q_{C 01: 23}+t_{D 2}+t_{D 3}$, then there exists an embedded normal sphere in $\mathcal{T}_{n}$, a contradiction. If no annuli of the form $\left(q_{C 01: 23}+q_{D 01: 23}+q_{E 01: 23}\right)$ exist in $S_{n+1} \cap \mathcal{B}_{3}$, then again there must be at least one piece of the form: $q_{C 01: 23}+q_{D 01: 23}+t_{E 2}+t_{E 3}$, $q_{D 01: 23}+q_{E 01: 23}+t_{C 2}+t_{C 3}$, and $q_{E 01: 23}+q_{C 01: 23}+t_{D 2}+t_{D 3}$, which completes the proof.

Although $q_{C 01: 23}+q_{D 01: 23}+t_{E 2}+t_{E 3}, q_{D 01: 23}+q_{E 01: 23}+t_{C 2}+t_{C 3}$, and $q_{E 01: 23}+q_{C 01: 23}+t_{D 2}+t_{D 3}$ are not in the image of embedded normal discs of $\mathcal{T}_{n}$, they are in the image of a pair of immersed normal discs. For example, $q_{A 03: 12}+q_{B 02: 13}+q_{A 01: 23}+q_{B 01: 23}=q_{C 01: 23}+q_{D 01: 23}+$ $t_{E 2}+t_{E 3}+t_{C 3}+t_{D 2}$. This is the key observation in the proof of the following lemma.

Proposition 12.9. Let $\mathcal{T}_{n}$ and $\mathcal{T}_{n+1, f}$ be the triangulations defined above, such that the exterior faces of $\mathcal{B}_{2}$ are not identified. If $\mathcal{T}_{n}$ admits a strict angle structure, then $\mathcal{T}_{n+1, f}$ is 1-efficient.

Proof. Suppose $\mathcal{T}_{n+1, f}$ is not 1-efficient. Then there is a closed embedded normal surface $S_{n+1}$ of $\mathcal{T}_{n+1, f}$ with non-negative Euler characteristic. 
Just as above, $S_{n+1} \cap \mathcal{B}_{3}$ must contain pieces of the form below, otherwise there is a closed embedded surface $S_{n}$ of $\mathcal{T}_{n}$ with non-negative Euler characteristic:

$q_{C 01: 23}+q_{D 01: 23}+t_{E 2}+t_{E 3}, q_{D 01: 23}+q_{E 01: 23}+t_{C 2}+t_{C 3}, q_{E 01: 23}+q_{C 01: 23}+t_{D 2}+t_{D 3}$, and $\left(q_{C 01: 23}+q_{D 01: 23}+q_{E 01: 23}\right)$.

By an identical argument to the one used in Proposition 12.8, we can rule out the annuli of the form $\left(q_{C 01: 23}+q_{D 01: 23}+q_{E 01: 23}\right)$. Thus, $S_{n+1} \cap \mathcal{B}_{3}$ contains exactly one of the following pairs of quads: $q_{C 01: 23}+q_{D 01: 23}+t_{E 2}+t_{E 3}, q_{D 01: 23}+q_{E 01: 23}+t_{C 2}+t_{C 3}$, or $q_{E 01: 23}+q_{C 01: 23}+t_{D 2}+t_{D 3}$.

Without loss of generality, assume it is $q_{C 01: 23}+q_{D 01: 23}+t_{E 2}+t_{E 3}$. Create a new surface $S_{n+1}^{\prime}$, by adding a boundary torus to $S_{n+1}$ until $S_{n+1} \cap \mathcal{B}_{3}$ contains the same number of copies of $\left(t_{C 3}+t_{D 2}\right)$ as $q_{C 01: 23}+q_{D 01: 23}+t_{E 2}+t_{E 3}$. Note that $\chi\left(S_{n+1}\right)=\chi\left(S_{n+1}^{\prime}\right)$. The image of $S_{n+1}^{\prime}$ under the 3-2 move, is an immersed normal surface $S_{n}$ with the same boundary arcs on $\mathcal{B}_{2}$ as $\partial \mathcal{B}_{3} \cap S_{n+1}^{\prime}$, with the property that for each copy of $q_{C 01: 23}+q_{D 01: 23}+t_{E 2}+t_{E 3}+\left(t_{C 3}+t_{D 2}\right)$ in $\mathcal{B}_{3} \cap S_{n+1}^{\prime}$ there is a copy of $q_{A 03: 12}+q_{B 02: 13}+q_{A 01: 23}+q_{B 01: 23}$. In the presence of any generalised angle structure on $T_{n}$, each quad is assigned an Euler characteristic, while the Euler characteristic of the triangles vanishes. Moreover, this Euler characteristic agrees with the Euler characteristic of $S_{n+1}^{\prime}$. However, if $\mathcal{T}_{n}$ admits a strict angle structure, then each quad has a negative contribution to Euler characteristic while each triangle does not contribute to Euler characteristic. This contradicts $\chi\left(S_{n+1}\right) \geq 0$.

Remark 12.10. The analogous statement to Proposition 12.9 for 0-2 moves does not hold. In fact, 'eLAkbbcdddhjac' has a degree 2 edge and is not 1-efficient. However, performing a 2-0 move along this edge results in the 1-efficient (in fact, geometric) triangulation of the figure 8 sister manifold, 'cPcbbbdxm'.

Example 12.11. We next provide an example of how a geometric triangulation can 'degenerate' to a triangulation which is not 1-efficient in two 2-3 moves: The triangulation 'eLAkbccddhhnqw' is geometric, the 2-3 move along face 5 gives 'fLAMcbccdeemejman' which does not admit a strict angle structure, and the 2-3 move along face 8 gives 'gLALQaccefffbgfgmqt' which is not 1-efficient.

However, perhaps the simplest example of a non 1-efficient triangulation arising from a 2-3 move on a 1-efficient triangulation has already been discussed in Table 5 . In fact the first Pachner move in that path a 2-3 move along face 2 of 'cPcbbbadh' resulting in 'dLQacccbgjs' breaks 1-efficiency. We again point out that there is an immersed normal surface in 'cPcbbbadh' determined by the quads $q_{0: 02: 13}, q_{0: 03: 12}, q_{1: 02: 13}$, and $q_{1: 02: 13}$ (face 2 is $0(023)=1(023)$ ), which maps to an embedded normal torus in 'dLQaccobjs'. (Note: Regina's labelling routine is different from that of the lists in this section.)

\section{Appendix A. Generalised Angle structures and the Euler Characteristic}

The relationship between angle structures and geometric structures is often described by saying that a generalised angle structure is a solution to the "linear part" of Thurston's gluing equations (see [Thu77, §4]). However, often this simplification involves only considering the edge equations, as in [LT08]. For the discussion below, we will pay special attention to the completeness conditions coming from the holonomies of the peripheral curves. 
Let $\mathcal{T}$ be an ideal triangulation with $n$ tetrahedra of a compact, orientable manifold 3manifold $M$ with boundary consisting of $r$ tori, and let $\square$ denote the set of all quad types in $\mathcal{T}$.

Recall, from Section 3.2, that a generalised angle structure on $\mathcal{T}$ is a function $\alpha: \square \rightarrow \mathbb{R}$ satisfying the equations (13) and (14), which represent the imaginary part of Thurston's logarithmic tetrahedral and edge equations (11), (10).

As described in [Thu77, §4.3.2], the logarithm of the derivative of the holonomy $H^{\prime}(\gamma)$ for each peripheral curve $\gamma$ must also vanish for a complete hyperbolic structure. Writing down these conditions for a pair of simple closed peripheral curves generating the fundamental group of each boundary component gives the logarithmic cusp equations (12).

The analogous condition for angle structures is defined as follows.

Definition A.1. Given a generalised angle structure $\alpha$ on an ideal triangulation $\mathcal{T}$, the rotational holonomy of a peripheral curve $\gamma$ is the imaginary part of the logarithm of the derivative of its holonomy: $\rho_{\alpha}(\gamma)=\operatorname{Im} \log H^{\prime}(\gamma)$.

If a generalised angle structure satisfies $\rho_{\alpha}(\gamma)=0$ for each peripheral curve $\gamma$, we say it has vanishing peripheral rotational holonomy.

More concretely, this means the vector $x=\left[\alpha\left(q_{j}\right) \alpha\left(q_{j}^{\prime}\right) \alpha\left(q_{j}^{\prime \prime}\right)\right] \in \mathbb{R}^{3 n}$ satisfies the linear equations given by the imaginary parts of Thurston's logarithmic edge, tetrahedral and completeness equations (10), (11), (12).

In the next section, we will discuss how to assign Euler characteristics to quad disks in the presence of a generalised angle structure.

Proposition A.2. Let $\mathcal{T}$ be an ideal triangulation of a compact manifold $M$ with boundary consisting of tori. Then there exist generalised angle structures on $\mathcal{T}$ with vanishing peripheral rotational holonomy.

Proof. We closely follow the argument for Lemma 10 in [LT08]. Assume that $\mathcal{T}$ has $n$ tetrahedra and $\partial M$ consists of $r$ tori. A vector $x \in \mathbb{R}^{3 n}$ defines a generalised angle structure with vanishing peripheral rotational holonomy if and only if

$$
A x=b,
$$

where $A$ is the $(2 n+2 r) \times 3 n$ matrix with rows $E_{i}, T_{j}, M_{k}, L_{k}$ and $b \in \mathbb{R}^{2 n+2 r}$ is the vector with $n$ entries $2 \pi$, followed by $n$ entries $\pi$, then $2 r$ zero entries.

From linear algebra, the equation $A x=b$ has a solution if and only if $b \in \operatorname{Im}(A)=$ $\operatorname{Ker}\left(A^{T}\right)^{\perp}$ if and only if

$$
z \in \mathbb{R}^{2 n+2 r} \text { and } A^{T} z=0 \Rightarrow z^{T} b=0 .
$$

Now $z=\left[\begin{array}{llll}x_{i} & y_{j} & p_{k} & q_{k}\end{array}\right]^{T} \in \operatorname{Ker}\left(A^{T}\right)$ if and only if $S=\sum_{i} x_{i} E_{i}+\sum_{j} y_{j} T_{j}+\sum_{k} p_{k} M_{k}+$ $\sum_{k} q_{k} L_{k}=0$, i.e. $S$ gives the trivial normal class in $Q(\mathcal{T} ; \mathbb{R})$. But this implies that $\partial S=0$ so $p_{k}=q_{k}=0$ for all $k$, hence $S=\sum_{i} x_{i} E_{i}+\sum_{j} y_{j} T_{j}=0$. Further $-\chi(S)=\sum_{i} 2 x_{i}+\sum_{j} y_{j}=0$, hence $z^{T} b=0$. This proves the result.

A.1. Euler characteristic from generalised angle structures. The following result shows how to compute the Euler characteristic of an embedded spun normal surface using 
any generalised angle structure. This result is well-known for closed normal surfaces; see for example [LT08, Lemma 15].

Proposition A.3. Let $\mathcal{T}$ be an ideal triangulation of a cusped 3-manifold $M$, and let $\alpha$ : $\square \rightarrow \mathbb{R}$ be a generalised angle structure on $\mathcal{T}$. Then the Euler characteristic of any embedded spun normal surface $S \in Q(\mathcal{T} ; \mathbb{R})$ is given by

$$
\chi(S)=-\sum_{q} \frac{\alpha(q) x_{q}}{\pi}+\frac{\rho_{\alpha}(\partial S)}{2 \pi},
$$

where the sum is over all normal classes $q$ in $\mathcal{T}, x_{q}$ is the normal coordinate of $q, \alpha(q) \in \mathbb{R}$ denotes the angle assigned to the two edges facing $q$, and $\rho_{\alpha}(\partial S)$ denotes the sum of the rotational holonomies of the boundary components of $S$, oriented as in $[\partial S]$.

This result follows from a combinatorial version of the Gauss-Bonnet theorem, as used by Thurston in [Thu77, Section 13.7]. Let $S$ be a compact surface, possibly with boundary and let $\mathcal{C}$ be a cell decomposition of $S$ into finitely many polygons. Then a formal angle structure (or combinatorial angle structure) on $\mathcal{C}$ is an assignment of real numbers ("angles") to the corners of the polygons such that the sum of angles is $2 \pi$ at each internal vertex.

We compute the Euler characteristic of $S$ as $\chi(S)=v-e+f$, where $v, e, f$ are the numbers of vertices, edges and faces in the cell decomposition. Now each $n$-gon $P$ contributes $\frac{1}{2 \pi}$ (sum of corner angles) to $v, n \times \frac{1}{2}=n / 2$ to $e$ and 1 to $f$, so contributes a local Euler characteristic

$$
\chi_{P}=\frac{1}{2 \pi}(\text { sum of corner angles in } P)-\frac{n}{2}+1
$$

to $\chi(S)=v-e+f$.

Adding up the terms $\chi_{P}$ over all polygons $P$ gives a contribution to $\chi(S)$ of +1 for each internal vertex, -1 for each internal edge and +1 for each face. Assume that there are also $k$ boundary vertices and, hence, $k$ boundary edges. Then to obtain $\chi(S)$ we need to add additional contributions of $\frac{1}{2 \pi}\left(2 \pi-\theta_{i}\right)$ where $\theta_{i}$ is the sum of internal angles at the $i$ th boundary vertex and $-1 / 2$ for each boundary edge. This gives

$$
\chi(S)=\sum_{P} \chi_{P}+\frac{1}{2 \pi} \sum_{i=1}^{k}\left(2 \pi-\theta_{i}\right)-\frac{k}{2}=\sum_{P} \chi_{P}+\frac{1}{2 \pi} \sum_{i=1}^{k}\left(\pi-\theta_{i}\right),
$$

and last term

$$
k_{g}(\partial S)=\sum_{i=1}^{k}\left(\pi-\theta_{i}\right)
$$

represents the total geodesic curvature of the boundary of $S$. Hence

Proposition A.4 (Combinatorial Gauss-Bonnet). Let $S$ be a compact surface with a finite cell decomposition $\mathrm{C}$ equipped with a formal angle structure. Then the Euler characteristic of $S$ is given by

$$
\chi(S)=\sum_{P} \chi_{P}+\frac{k_{g}(\partial S)}{2 \pi},
$$

summed over all polygons $P$, where $\chi_{P}$ is defined by (35) and where $k_{g}(\partial S)$ denotes the total geodesic curvature of $\partial S$. 
Proof of Proposition A.3. Let $S$ be a (non-compact) embedded spun normal surface in $\mathcal{T}$ and choose a compact subsurface $S_{0}$ consisting of all quads and a finite number of normal triangles such that $S \backslash S_{0}$ is a collection of annuli spiralling out to the cusps of $M$. By adding an additional layer of triangles, if necessary, we can assume that there is a neighbourhood of $\partial S_{0}$ consisting entirely of triangles. Projecting these normal triangles to a horosphere gives a layer of Euclidean triangles to the left of the boundary, and the rotational holonomy of $\partial S_{0}$ is the sum

$$
\rho_{\alpha}(\partial S)=\rho_{\alpha}\left(\partial S_{0}\right)=\sum_{i=1}^{k}\left(\pi-\theta_{i}\right),
$$

where $\theta_{i}$ is the sum of triangle angles at the $i$ th vertex on the boundary.

Now $S_{0}$ has a cell decomposition into quads and triangles, and a generalised angle structure $\alpha$ on $\mathcal{T}$ assigns an "angle" (in $\mathbb{R}$ ) to each corner of each 2-cell in this decomposition, giving a formal angle structure on this cell decomposition. Now each 2-cell gives a contribution to the Euler characteristic as follows:

- Each quad $q$ contributes $\chi(q)=\frac{1}{2 \pi}$ (angle sum) - 1. But the sum of angles in the quad is $2 \pi$ minus twice the angle $\alpha(q)$ on the two edges facing $q$, so we have $\chi(q)=-\alpha(q) / \pi$.

- Each triangle $t$ contributes $\chi(t)=\frac{1}{2 \pi}$ (angle sum) $2-3 / 2+1=0$.

Hence the combinatorial Gauss-Bonnet theorem gives

$$
\chi(S)=\chi\left(S_{0}\right)=-\sum_{q} \frac{\alpha(q) x_{q}}{\pi}+\frac{1}{2 \pi} \sum_{i=1}^{k}\left(\pi-\theta_{i}\right)=-\sum_{q} \frac{\alpha(q) x_{q}}{\pi}+\frac{\rho_{\alpha}(\partial S)}{2 \pi} .
$$

Since the boundary term vanishes for any angle structure with vanishing peripheral rotational holonomy, this gives the following convenient way of computing the Euler characteristic:

Corollary A.5. Let $\mathcal{T}$ be an ideal triangulation of a cusped 3-manifold $M$, and let $\alpha: \square \rightarrow \mathbb{R}$ be a generalised angle structure on $\mathcal{T}$ with vanishing peripheral rotational holonomy. Then the Euler characteristic of any embedded spun normal surface $S \in Q(\mathcal{T} ; \mathbb{R})$ is given by

$$
\chi(S)=-\sum_{q} \frac{\alpha(q) x_{q}}{\pi} .
$$

Definition A.6. For a general Q-normal class $S \in Q(\mathcal{T} ; \mathbb{R})$ we take the formula (34) as the definition of the formal Euler characteristic of $S$. This is a linear function $\chi: Q(\mathcal{T} ; \mathbb{R}) \rightarrow \mathbb{R}$.

Remark A.7. The independence of choice of angle structure follows directly from [LT08, Lemma 15]. For the angle structures in this paper, $(A, \kappa)=(0,0)$ and our $\alpha(q)$ is equal to one half of $A(q)$ as in [LT08].

Another consequence of these results is 
Corollary A.8. Each peripheral curve solution has vanishing formal Euler characteristic. Hence

$$
\chi\left(\sum_{i} x_{i} E_{i}+\sum_{i} y_{j} T_{j}+\sum_{k}\left(p_{k} M_{k}+q_{k} L_{k}\right)\right)=-\sum_{i} 2 x_{i}-\sum_{j} y_{j},
$$

since $\chi\left(E_{i}\right)=-2$ and $\chi\left(T_{j}\right)=-1$.

Proof. Let $\alpha$ be a generalised angle structure on $\mathcal{T}$, and let $S$ be the peripheral curve solution given by a curve with homology class $c \in H_{1}(\partial M ; \mathbb{Z})$. Then we have $\partial S=2 c$ (see Section 7.1). and, by definition, $\rho_{\alpha}(c)=\sum_{q} \alpha(q) x_{q}$ where $x_{q}$ are the quad coordinates of $S$. Hence

$$
\chi(S)=-\sum_{q} \frac{\alpha(q) x_{q}}{\pi}+\frac{\rho_{\alpha}(\partial S)}{2 \pi}=-\frac{\rho_{\alpha}(c)}{\pi}+\frac{\rho_{\alpha}(\partial S)}{2 \pi}=0 .
$$

Remark A.9. We could also use the formula (39) to define the formal Euler characteristic of a general $Q$-normal class $S \in Q(\mathcal{T} ; \mathbb{R})$.

Example A.10. For the figure eight knot complement with its triangulation given in Example 4.1, the angle structure equations have general solution of the form

$$
\alpha=\left(\alpha_{0}+t_{1} \alpha_{1}+t_{2} \alpha_{2}+t_{3} \alpha_{3}\right) \pi \text {, with } t_{i} \in \mathbb{R}
$$

where $\alpha_{0}=(-1,2,0,1,0,0), \alpha_{1}=(2,-2,0,-1,0,1), \alpha_{2}=(1,-1,0,-1,1,0), \alpha_{3}=(1,-2,1,0,0,0)$. This gives rotational holonomies for the meridian and longitude

$$
\rho_{\alpha}(M)=\left(-1+t_{1}+t_{2}+t_{3}\right) \pi \text { and } \rho_{\alpha}(L)=\left(2-4 t_{1}-2 t_{2}\right) \pi .
$$

Now there is an embedded spun normal once punctured Klein bottle with quad coordinates $K=(0,0,2,0,1,0)$ with boundary $\partial K=4 \mu+1 \lambda$. The general formula (34) for the Euler characteristic gives

$\chi(K)=-\sum_{q} \frac{\alpha(q)}{\pi}+\frac{\rho_{\alpha}(\partial S)}{2 \pi}=-\frac{1}{\pi} \alpha \cdot K+\frac{1}{2 \pi} \alpha \cdot(4 M+L)=\left(-t_{2}-2 t_{3}\right)+\left(-1+t_{2}+2 t_{3}\right)=-1$

as expected.

Alternatively we can write $K=2 M+\frac{1}{2} L+1 T_{2}$, hence $\chi(K)=-1$ by formula (39).

In fact, finding angle structures can be seen as a dual problem to the existence of surfaces of non-negative Euler characteristic. To make this precise, we first remind the reader of a key tool, Farkas' Lemma.

Theorem A.11 (Farkas' Lemma). If $A$ is a real $m \times n$ matrix and $b \in \mathbb{R}^{m}$, and the standard Euclidean inner product on $\mathbb{R}^{m}$, then the following holds:

$\left\{x \in \mathbb{R}^{n} \mid A x=b, x>0\right\}$ is non-empty if and only if for all $z \in \mathbb{R}^{m}$ such that $A^{T} z \neq 0$ and $A^{T} z \geq 0, z \cdot b>0$.

Here $x \geq 0$ (respectively $x>0$ ) indicates that all coordinates of $x$ are non-negative (respectively positive). 
By using Farkas' Lemma, we can mimic the above argument for Theorem A.2 to get necessary and sufficient conditions for the existence of strict angle structures with vanishing peripheral rotational holonomy.

Proposition A.12. Let $\mathcal{T}$ be an ideal triangulation of a compact manifold $M$ with boundary consisting of tori. Then there exist a strict angle structure on $\mathcal{T}$ with vanishing peripheral rotational holonomy if and only if there exists no $Q$-normal class $0 \neq Q \in Q\left(\mathcal{T} ; \mathbb{R}_{+}\right)$with $\chi(S) \geq 0$ if and only if $0 \neq Q \in Q\left(\mathcal{T} ; \mathbb{R}_{+}\right)$implies $\chi(S)<0$.

Proof. We closely follow the argument of Theorem A.2 (and Lemma 10 in [LT08]). Using the notation from the proof of Theorem A.2, a vector $x \in \mathbb{R}^{3 n}$ defines a strict angle structure with vanishing peripheral rotational holonomy if and only if

$$
A x=b \text { and } x>0 .
$$

By Farkas' Lemma, this is equivalent to the condition that

$$
\text { for all } z \in \mathbb{R}^{2 n+2 r} \text { such that } A^{T} z \neq 0 \text { and } A^{T} z \geq 0, z \cdot b>0 \text {. }
$$

Now if $z=\left[\begin{array}{llll}x_{i} & y_{j} & p_{k} & q_{k}\end{array}\right]$ then $S=A^{T} z=\sum_{i} x_{i} E_{i}+\sum_{j} y_{j} T_{j}+\sum_{k} p_{k} M_{k}+\sum_{k} q_{k} L_{k}$ represents a Q-normal class in $Q(\mathcal{T} ; \mathbb{R})$. But $A^{T} z \neq 0$ and $A^{T} z \geq 0$ iff $S \neq 0$ and $S$ has all quad coordinates $\geq 0$, i.e. $S \in Q\left(\mathcal{T} ; \mathbb{R}_{+}\right)$. Further, $z \cdot b=\sum_{i} 2 x_{i}+\sum_{j} y_{j}=-\chi(S)$. So the result follows.

\section{REFERENCES}

[Bac] David Bachman, Normalizing topologically minimal surfaces ii: Disks, arXiv:1210.4574, Preprint 2012.

[Ber91] John Berge, The knots in $D^{2} \times S^{1}$ which have nontrivial Dehn surgeries that yield $D^{2} \times S^{1}$, Topology Appl. 38 (1991), no. 1, 1-19.

[BP97] Riccardo Benedetti and Carlo Petronio, Branched standard spines of 3-manifolds, Lecture Notes in Mathematics, vol. 1653, Springer-Verlag, Berlin, 1997.

[Bur] Benjamin Burton, Regina, software for 3-manifold topology and normal surface theory, http: //regina. sourceforge.net (30/01/2015).

[Bur11] Benjamin A. Burton, The Pachner graph and the simplification of 3-sphere triangulations, Computational geometry (SCG'11), ACM, New York, 2011, pp. 153-162.

[CDW] Marc Culler, Nathan M. Dunfield, and Jeffrey R. Weeks, SnapPy, a computer program for studying the topology of 3-manifolds, Available at http://snappy . computop.org (30/01/2015).

[Cho06] Young-Eun Choi, Neumann and Zagier's symplectic relations, Expo. Math. 24 (2006), no. 1, $39-51$.

[DD] Blake Dadd and Aochen Duan, Constructing infinitely many geometric triangulations of the figure eight knot complement, arXiv:1508.04942, Preprint 2015.

[DG12] Nathan M. Dunfield and Stavros Garoufalidis, Incompressibility criteria for spun-normal surfaces, Trans. Amer. Math. Soc. 364 (2012), no. 11, 6109-6137.

[DGG13] Tudor Dimofte, Davide Gaiotto, and Sergei Gukov, 3-manifolds and 3d indices, Adv. Theor. Math. Phys. 17 (2013), no. 5, 975-1076.

[DGG14]__ Gauge theories labelled by three-manifolds, Comm. Math. Phys. 325 (2014), no. 2, 367419.

[FKB08] Charles Frohman and Joanna Kania-Bartoszynska, The quantum content of the normal surfaces in a three-manifold, J. Knot Theory Ramifications 17 (2008), no. 8, 1005-1033.

[FM09] Evgeny Fominykh and Bruno Martelli, k-normal surfaces, J. Differential Geom. 82 (2009), no. 1, $101-114$. 
[Gab89] David Gabai, Surgery on knots in solid tori, Topology 28 (1989), no. 1, 1-6.

[Gar15] Stavros Garoufalidis, The 3D index of an ideal triangulation and angle structures, 2015, arXiv: 1208.1663.

[GHRS15] Stavros Garoufalidis, Craig D. Hodgson, J. Hyam Rubinstein, and Henry Segerman, 1-efficient triangulations and the index of a cusped hyperbolic 3-manifold, Geom. Topol. 19 (2015), no. 5, 2619-2689.

[Hak61a] Wolfgang Haken, Ein Verfahren zur Aufspaltung einer 3-Mannigfaltigkeit in irreduzible 3Mannigfaltigkeiten, Math. Z. 76 (1961), 427-467.

[Hak61b] _ , Theorie der Normalflächen, Acta Math. 105 (1961), 245-375.

[Hak68] _ Some results on surfaces in 3-manifolds, Studies in Modern Topology, Math. Assoc. Amer. (distributed by Prentice-Hall, Englewood Cliffs, N.J.), 1968, pp. 39-98.

[Har] Robert Haraway, Determining hyperbolicity of compact orientable 3-manifolds with torus boundary, arXiv:1410.7115, Preprint 2014.

[Hat] Alan Hatcher, Notes on basic 3-manifold topology, url\{https://www.math.cornell.edu/ hatcher/ 3M/3Mdownloads.html , 2000.

[JR03] William Jaco and J. Hyam Rubinstein, 0-efficient triangulations of 3-manifolds, J. Differential Geom. 65 (2003), no. 1, 61-168.

[Kan05] Ensil Kang, Normal surfaces in non-compact 3-manifolds, J. Aust. Math. Soc. 78 (2005), no. 3, 305-321.

[Kne29] Hellmuth Kneser, Geschlossene flächen in dreidimensionalen mannigfaltigkeiten, Jahresber. Deutsch. Math.-Verein. 38 (1929), 248-260.

[KR04] Ensil Kang and J. Hyam Rubinstein, Ideal triangulations of 3-manifolds. I. Spun normal surface theory, Proceedings of the Casson Fest, Geom. Topol. Monogr., vol. 7, Geom. Topol. Publ., Coventry, 2004, pp. 235-265.

[KR05] Ideal triangulations of 3-manifolds. II. Taut and angle structures, Algebr. Geom. Topol. 5 (2005), 1505-1533.

[Lac00] Marc Lackenby, Taut ideal triangulations of 3-manifolds, Geom. Topol. 4 (2000), 369-395 (electronic).

[LT08] Feng Luo and Stephan Tillmann, Angle structures and normal surfaces, Trans. Amer. Math. Soc. 360 (2008), no. 6, 2849-2866.

[Mat87] S. V. Matveev, Transformations of special spines, and the Zeeman conjecture, Izv. Akad. Nauk SSSR Ser. Mat. 51 (1987), no. 5, 1104-1116, 1119.

[Mat03] Sergei Matveev, Algorithmic topology and classification of 3-manifolds, Algorithms and Computation in Mathematics, vol. 9, Springer-Verlag, Berlin, 2003.

[Mon73] José M. Montesinos, Seifert manifolds that are ramified two-sheeted cyclic coverings, Bol. Soc. Mat. Mexicana (2) 18 (1973), 1-32.

[MP06] Bruno Martelli and Carlo Petronio, Dehn filling of the "magic" 3-manifold, Comm. Anal. Geom. 14 (2006), no. 5, 969-1026.

[Neu92] Walter D. Neumann, Combinatorics of triangulations and the Chern-Simons invariant for hyperbolic 3-manifolds, Topology '90 (Columbus, OH, 1990), Ohio State Univ. Math. Res. Inst. Publ., vol. 1, de Gruyter, Berlin, 1992, pp. 243-271.

[NZ85] Walter D. Neumann and Don Zagier, Volumes of hyperbolic three-manifolds, Topology 24 (1985), no. 3, 307-332.

[Pie88] Riccardo Piergallini, Standard moves for standard polyhedra and spines, Rend. Circ. Mat. Palermo (2) Suppl. (1988), no. 18, 391-414, Third National Conference on Topology (Italian) (Trieste, 1986).

[Rub97] J. H. Rubinstein, Polyhedral minimal surfaces, Heegaard splittings and decision problems for 3dimensional manifolds, Geometric topology (Athens, GA, 1993), AMS/IP Stud. Adv. Math., vol. 2, Amer. Math. Soc., Providence, RI, 1997, pp. 1-20.

[Sto00] Michelle Stocking, Almost normal surfaces in 3-manifolds, Trans. Amer. Math. Soc. 352 (2000), no. $1,171-207$. 
[Thu77] William Thurston, The geometry and topology of 3-manifolds, Universitext, Springer-Verlag, Berlin, 1977, http://msri.org/publications/books/gt3m.

[Til08] Stephan Tillmann, Normal surfaces in topologically finite 3-manifolds, Enseign. Math. (2) 54 (2008), no. 3-4, 329-380.

[Tol98] Jeffrey L. Tollefson, Normal surface Q-theory, Pacific J. Math. 183 (1998), no. 2, 359-374.

[Wal11] Genevieve S. Walsh, Incompressible surfaces and spunnormal form, Geom. Dedicata 151 (2011), 221-231.

School of Mathematics, Georgia Institute of Technology, Atlanta, Georgia 30332-0160, USA

E-mail address: stavros@math.gatech.edu

School of Mathematics and Statistics, The University of Melbourne, Parkville, ViC, 3010, AUSTRALIA

E-mail address: craigdh@unimelb.edu.au

School of Mathematics and Statistics, The University of Melbourne, Parkville, ViC, 3010, AUSTRALIA

E-mail address: nhoffman@ms.unimelb.edu.au

School of Mathematics and Statistics, The University of Melbourne, Parkville, ViC, 3010, AUSTRALIA

E-mail address: rubin@ms.unimelb.edu.au 